Saclay-T97/023, DESY 97-046

\title{
Onium-Onium scattering at fixed impact parameter: exact equivalence between the color dipole model and the BFKL Pomeron.
}

\author{
Henri Navelet \\ Service de Physique Théoriqu用, CE-Saclay \\ 91191 Gif-sur-Yvette, France \\ Samuel Wallon [ \\ II. Institut für Theoretische Physik, Universität Hamburg \\ Hamburg, Germany
}

\begin{abstract}
We compute the onium-onium scattering amplitude at fixed impact parameter in the framework of the perturbative QCD dipole model. Relying on conformal properties of the dipole cascade and of the elementary dipole-dipole scattering amplitude, we obtain an exact result for this onium-onium scattering amplitude, which is proven to be identical to the BFKL result, and which exhibits the frame invariance of the calculation. The asymptotic expression for this amplitude and for the dipole distribution in an onium at fixed impact parameter agree with previous numerical simulations. We show how it is possible to describe onium- $e^{ \pm}$deep inelastic scattering in the dipole model, relying on $k_{T}$-factorization properties. The elementary scattering amplitudes involved in the various processes are computed using eikonal techniques.
\end{abstract}

*Laboratoire de la Direction des Sciences de la Matière du Commissariat à l'Energie Atomique

$\dagger$ Alexander von Humboldt Fellow 



\section{Introduction}

The HERA experiments [1, 2] have focused attention on the Balitsky-Fadin- Kuraev-Lipatov (BFKL) Pomeron [3], which should be relevant for describing the small- $x_{b j}$ behaviour of the proton structure function. More generally, this perturbative QCD hard Pomeron describes the behaviour of hadronic scattering amplitudes at very high energy $s$ and fixed momentum transfer $t \sim-m^{2}$ ( $m$ being an hadronic mass scale). In this Leading Logarithmic Approximation, one takes into account the exchange of a bound state of two reggeized gluons in $t$-channel. This resummation, based on perturbative Regge physics, predicts an increase of the amplitude

$$
A_{B F K L} \propto s^{\alpha_{P}}
$$

where

$$
\alpha_{P}=1+\frac{\alpha_{S} N_{c}}{\pi} 4 \ln 2>1
$$

Using the optical theorem, it follows that this behaviour violates the Froissart bound at very high $s$

$$
\sigma_{t o t} \leq c \ln ^{2} s .
$$

This violation is directly related to the unitarization problem of QCD, which is one of the main problems to be solved in the theory of strong interaction. Various approaches have been recently proposed in order to restore unitarity. In the multiregge approach, it has been shown that the Generalized Leading Logarithmic Approximation [4, 5, 6], where one takes into account the exchange of any fixed number of reggeized gluons in $t$-channel, is equivalent to the noncompact Heisenberg XXX spin chain [7, 8] in the multicolor limit of QCD. The solution of this integrable model is still an open problem [9, 10, 11, 12, 13. In the model recently developped by Mueller et al [14, 15, 16, 17] and separately by Nikolaev et al [18, 19], in order to control the perturbative approach, one deals with onia, which are heavy quark-antiquark bound states, so that their transverse size naturally provides an infra-red cut-off. The relevant degrees of freedom at high energy are then made of color dipoles. These color dipoles produce a classical cascade in the multicolor limit, which reveals a Pomeron type dynamics. This approach, combined with $k_{T}$-factorization [20, 21, 22], has been successfully applied to deep inelastic $e^{ \pm}-p$ scattering for describing HERA data for $F_{2}$ [23, 24, 25]. In the more general case of onium-onium scattering, the BFKL approximation corresponds to the exchange of one pair of gluons between two excited dipoles, each one being extracted from one of the two onia. The unitarization problem can also be studied in this dipole model. In real physics, unitarity and analyticity of the $S$ matrix and the finite range of strong interaction lead to the Froissart bound (1.3) for total cross-section. Unitarity implies in particular at fixed impact parameter that the probability of any event cannot exceed 1 , that is

$$
|S(b)| \leq 1
$$

One way of enforcing unitarity in the framework of perturbative QCD would then be to take into account two and more left moving dipoles scattering off an equal number of right moving dipoles. As long as the relative rapidity $Y$ of the two onia is not too high, it is possible to deal with unitarization effects without facing saturations effects, that is one can consider each dipole in the wave function of the onia as still dilute [16, 26]. However, because of non-trivial dynamics in transverse space, the usual Glauber multiple scattering series, which corresponds to summing up multiple Pomeron exchanges, diverges factorially. A possible way of escaping this problem is to sum over to the number of exchanged Pomerons, and only after this summation is performed, 
averaging over configurations of dipoles in the onia wave functions. Such an approach, except for a toy model where the transverse dynamics is absent [16], has not been yet carried out analytically. Numerically, such an approach is possible, and Monte Carlo simulations indeed show that QCD unitarizes in the dipole framework [27, 28].

Our aim is to study how this can be carried out analytically. In this paper, we will focus on the onium-onium cross-section at fixed impact parameter. It is organized as follows. In section 2, we calculate the onium-onium scattering amplitude at fixed impact parameter. The method developped here is rather general, and is based on expansions over conformal three points correlation functions. Using this representation, we calculate the distribution of dipoles inside an onium, at fixed impact parameter, and obtain its precise dependence with respect to the transverse size of the excited dipole. Then, the cross-section at fixed impact parameter is obtained using the elementary dipole-dipole cross-section, which is calculated by eikonal methods and expanded on a conformal basis. Our result shows the exact equivalence between the dipole and the BFKL approaches for inclusive processes. In section 3, we study some physical applications of the dipole picture, namely $e^{ \pm}-\mathcal{O}$ nium deep inelastic scattering. We rely on $k_{T}$-factorization and apply eikonal techniques for computing the elementary dipole-gluon cross-section.

\section{Onium-onium cross-section at fixed impact parameter}

In this section, we calculate the onium-onium scattering amplitude in the BFKL approximation. This calculation has been first carried out in Ref. [15, 16]. It used an expansion on a conformal three points correlation function basis. Asymptotic expressions of this basis were used in this calculation. The calculation which is presently developped gives the correct dependence with respect to the transverse sizes of onia. This dependence is highly non trivial and could not be obtained without a careful treatment of the conformal basis.

We thus consider onium-onium scattering in the leading logarithmic approximation. The infinite-momentum wave function of an onium, in the large $N_{c}$ limit, was calculated perturbatively in Ref. [14]. This calculation is based on an approximation of eikonal type, due to the ordering of longitudinal momenta. This allows one to compute the probability of emitting a gluon from a quark, and then from a quark-antiquark pair, that is from a color dipole. In the multicolor limit, the cascade of emitted soft gluons decouples, leading to a semi-classical cascade of color dipoles, in term of probability, since interference terms cancels in this limit. The onium-onium elastic scattering amplitude at fixed impact parameter $A(Y, b)$ can then be expressed using a parton type formulation. It involves the number of dipoles in each onium and the elementary cross-section of two such dipoles. For a relative rapidity $Y$ and impact parameter $b, A(Y, \underline{b})$ can be expressed as

$$
A(Y, \underline{b})=-i \int d^{2} \underline{x}_{1} \int d^{2} \underline{x}_{2} \int_{0}^{1} d z_{1} \int_{0}^{1} d z_{2} \Phi\left(\underline{x}_{1}, z_{1}\right) \Phi\left(\underline{x}_{2}, z_{2}\right) F\left(\underline{x}_{1}, \underline{x}_{2}, \tilde{Y}, \underline{b}\right) .
$$

$\Phi\left(\underline{x}_{i}, z_{i}\right)$ is the square of the heavy quark-antiquark part of the onium wavefunction, $\underline{x}_{i}$ being the transverse size of the quark-antiquark pair and $z_{i}$ the longitudinal momentum fraction of the antiquark. The momentum $p_{\tilde{Y}}^{+}$and $p_{2}^{-}$of the two onia are supposed to be large, with $\underline{p}_{1}=\underline{p}_{2}=0 . Y$ is related to $\tilde{Y}$ by $\tilde{Y}=Y+\ln z_{1} z_{2}$, due to the fact that the perturbative dipole cascade originates from the quark-antiquark pair. The distribution $\Phi(\underline{x}, z)$ of this pair cannot be computed perturbatively, and goes far beyond the purpose of the present approach. The 
scattering amplitude $F$ is then evaluated in term of the perturbative dipole cascade. Following Ref. [14, 15], we define $n\left(x_{01}, x, b, \tilde{Y}\right)$ such that

$$
N(\underline{x}, \underline{b}, Y)=\int d^{2} \underline{x} \int_{0}^{1} d z_{1} \Phi\left(\underline{x}, z_{1}\right) n\left(\underline{x}, \underline{x}^{\prime}, \underline{Y}, \underline{b}\right)
$$

is the number density of dipoles of transverse size $\underline{x}^{\prime}$, at a tranverse distance $\underline{b}$ from the center of the quark-antiquark pair, where the momentum fraction of the softest of the two gluons (or quark or antiquark) which compose the dipole is larger or equal to $e^{-Y} . \tilde{Y}$ is the relative rapidity with respect to the heavy quark given by $\tilde{Y}=Y+\ln z_{1}$. In the leading logarithm approximation (noted $F^{(1)}$ ) where the scattering is due to the exchange of a single pair of gluons between the two dipoles extracted from the left and right moving onia, $F^{(1)}$ reads

$$
\begin{aligned}
& F^{(1)}\left(\underline{x}_{1}, \underline{x}_{2}, \tilde{Y}, \underline{b}\right)=-\frac{1}{2} \int \frac{d^{2} \underline{x}_{1}^{\prime}}{2 \pi x_{1}^{\prime 2}} \frac{d^{2} \underline{x}_{2}^{\prime}}{2 \pi x_{2}^{\prime 2}} d^{2} \underline{b}_{1} d^{2} \underline{b}_{2} d^{2}\left(\underline{b}_{2}^{\prime}-\underline{b}_{1}^{\prime}\right) \delta^{2}\left(\underline{b}_{1}-\underline{b}_{2}-\underline{b}_{1}^{\prime}+\underline{b}_{2}^{\prime}-\underline{b}\right) \\
& \quad \times n\left(\underline{x}_{1}, \underline{x}_{1}^{\prime}, \tilde{Y}_{1}, \underline{b}_{1}\right) n\left(\underline{x}_{2}, \underline{x}_{2}^{\prime}, \tilde{Y}_{2}, \underline{b}_{2}\right) \sigma_{D D}\left(\underline{x}_{1}^{\prime}, \underline{x}_{2}^{\prime}, \underline{b}_{1}^{\prime}-\underline{b}_{2}^{\prime}\right) .
\end{aligned}
$$

The rapidities $\tilde{Y}_{1}$ and $\tilde{Y}_{2}$ are such that $\tilde{Y}=\tilde{Y}_{1}+\tilde{Y}_{2}$. Eq. (2.7) involves the elementary dipoledipole cross-section at fixed impact parameter $\sigma_{D D}$, which has been evaluated in [15], and which is calculated in appendix A.2 using eikonal techniques. For two dipoles of transverse sizes $\underline{x}_{1}^{\prime}$ and $\underline{x}_{2}^{\prime}$, whose centers are located at $\underline{b}_{1}^{\prime}$ and $\underline{b}_{2}^{\prime}$, one obtains

$$
\sigma_{D D}\left(\underline{x}_{1}^{\prime}, \underline{x}_{2}^{\prime}, \underline{b}_{1}^{\prime}-\underline{b}_{2}^{\prime}\right)=\alpha_{s}\left\{\ln \frac{\left|\underline{b}_{1}^{\prime}-\underline{b}_{2}^{\prime}+\frac{\underline{x}_{1}^{\prime}+\underline{x}_{2}^{\prime}}{2}\right|\left|\underline{b}_{1}^{\prime}-\underline{b}_{2}^{\prime}-\frac{\underline{x}_{1}^{\prime}+\underline{x}_{2}^{\prime}}{2}\right|}{\left|\underline{b}_{1}^{\prime}-\underline{b}_{2}^{\prime}+\frac{\underline{x}_{1}^{\prime}-\underline{x}_{2}^{\prime}}{2}\right|\left|\underline{b}_{1}^{\prime}-\underline{b}_{2}^{\prime}-\frac{\underline{x}_{1}^{\prime}-\underline{x}_{2}^{\prime}}{2}\right|}\right\}^{2} .
$$

The forward scattering amplitude can then be evaluated by integration over impact parameter, namely

$$
A(Y)=\int d^{2} \underline{b} A(Y, \underline{b}) .
$$

$A(Y)$ is normalized so that the optical theorem reads

$$
\sigma(Y)=2 \operatorname{Im} A \text {. }
$$

In order to get the expression for $n\left(\underline{x}, \underline{x^{\prime}}, \tilde{Y}, \underline{b}\right)$, one relies on the global conformal invariance of the dipole emission kernel, related to the absence of scale. We thus decompose this distribution on the basis of conformaly invariant three points holomorphic and antiholomorphic correlation functions [29, 30]. Introducing complex coordinates in the two-dimensional transverse space

$$
\begin{aligned}
& \underline{\rho}=\left(\rho_{x}, \rho_{y}\right) \\
& \rho=\rho_{x}+i \rho_{y} \text { and } \rho^{*}=\rho_{x}-i \rho_{y},
\end{aligned}
$$

the complete set of eigenfunctions $E^{n, \nu}$ of the dipole emission kernel is

$$
E^{n, \nu}\left(\underline{\rho}_{10}, \underline{\rho}_{20}\right)=(-1)^{n}\left(\frac{\rho_{12}}{\rho_{10} \rho_{20}}\right)^{h}\left(\frac{\rho_{12}^{*}}{\rho_{10}^{*} \rho_{20}^{*}}\right)^{\bar{h}}
$$

with

$$
\begin{aligned}
h & =\frac{1-n}{2}+i \nu \\
\bar{h} & =\frac{1+n}{2}+i \nu
\end{aligned}
$$


being the corresponding conformal weights, with $n$ integer and $\nu$ real. This set constitutes a unitary irreducible representation of $\mathrm{SL}(2, \mathbf{C})$ [31].

We get rid of the longitudinal degrees of freedom by using a Mellin transform with respect to $\tilde{Y}$, namely

$$
n\left(x, x^{\prime}, \tilde{Y}, b\right)=\int \frac{d \omega}{2 i \pi} e^{\omega \tilde{Y}} n_{\omega}\left(x, x^{\prime}, b\right) .
$$

Expanding the dipole distribution on the conformal basis, one writes

$$
\begin{aligned}
n_{\omega}(x, & \left.x^{\prime}, b\right)=\sum_{n=-\infty}^{n=+\infty} 8 \int \frac{d \nu}{(2 \pi)^{3}} \frac{d^{2} w}{x^{\prime 2}}\left(\nu^{2}+\frac{n^{2}}{4}\right) n_{\{\nu, n\} \omega} \\
\times E^{n, \nu} & \left(\underline{b}+\frac{\underline{x}^{\prime}}{2}-\underline{w}, \underline{b}-\frac{\underline{x}^{\prime}}{2}-\underline{w}\right) E^{n, \nu *}\left(\frac{\underline{x}}{2}-\underline{w},-\frac{\underline{x}}{2}-\underline{w}\right) .
\end{aligned}
$$

In this expression, the transverse integration is done with respect to the coordinate $\underline{w}$ of the center of mass of the quark-antiquark pair. The longitudinal dynamics gives rise to the term $n_{\{\nu, n\} \omega}$, which was computed in Ref. [14], and has the following expression

$$
n_{\{\nu, n\} \omega}=\frac{2}{\omega-\frac{2 \alpha_{S} N_{c}}{\pi} \chi(n, \nu)},
$$

where

$$
\chi(n, \nu)=\psi(1)-\frac{1}{2} \psi\left(\frac{|n|+1}{2}+i \nu\right)-\frac{1}{2} \psi\left(\frac{|n|+1}{2}-i \nu\right)=\psi(1)-\operatorname{Re} \psi\left(\frac{|n|+1}{2}+i \nu\right) .
$$

Going back to the longitudinal space, the distribution of dipoles takes the form

$$
\begin{aligned}
n\left(\underline{x}, \underline{x}^{\prime}, \tilde{Y}, \underline{b}\right)=\sum_{n=-\infty}^{+\infty} & 16 \int \frac{d \nu}{(2 \pi)^{3}} \frac{d^{2} \underline{w}}{x^{\prime 2}}\left(\nu^{2}+\frac{n^{2}}{4}\right) \exp \left(\frac{2 \alpha N_{c}}{\pi} \chi(n, \nu) \tilde{Y}\right) \\
& \times E^{n, \nu}\left(\underline{b}+\frac{\underline{x}^{\prime}}{2}-\underline{w}, \underline{b}-\frac{\underline{x}^{\prime}}{2}-\underline{w}\right) E^{n, \nu *}\left(\frac{\underline{x}}{2}-\underline{w},-\frac{\underline{x}}{2}-\underline{w}\right) .
\end{aligned}
$$

This distribution can be more easily computed by using a Fourier transform with respect to the impact parameter, that is by fixing the $t$ channel exchanged momentum. We thus define

$$
n\left(\underline{x}, \underline{x}^{\prime}, \tilde{Y}, \underline{b}\right)=\int \frac{d^{2} \underline{q}}{(2 \pi)^{2}} e^{-i \underline{q} \cdot \underline{b}} n\left(\underline{x}, \underline{x}^{\prime}, \tilde{Y}, \underline{q}\right),
$$

and introduce, following Ref. [29], the corresponding mixed representation of $E^{n, \nu}$, namely

$$
E_{q}^{n, \nu}(\rho)=\frac{2 \pi^{2}}{b_{n, \nu}} \int \frac{d^{2} \underline{R}}{|\rho|} e^{i q} \cdot \underline{R} E^{n, \nu}\left(\underline{R}+\frac{\rho}{2}, \underline{R}-\frac{\rho}{2}\right) .
$$

The normalisation term $b_{n, \nu}$ is given by

$$
b_{n, \nu}=\pi^{3} \frac{1}{-i \nu+|n| / 2} 2^{4 i \nu} \frac{\Gamma(-i \nu+(1+|n|) / 2)}{\Gamma(i \nu+(1+|n|) / 2)} \frac{\Gamma(i \nu+|n| / 2)}{\Gamma(-i \nu+|n| / 2)} .
$$

In this Fourier representation the dipole distribution reads

$$
n\left(\underline{x}, \underline{x}^{\prime}, \tilde{Y}, \underline{b}\right)=\sum_{n=-\infty}^{+\infty} \int \frac{d \nu}{2 \pi} \int \frac{d^{2} \underline{q}}{(2 \pi)^{2}} e^{-i \underline{q} \cdot \underline{b}} E_{q}^{n, \nu *}(x) E_{q}^{n, \nu}\left(x^{\prime}\right) \frac{x}{x^{\prime}} \exp \left(\frac{2 \alpha N_{c}}{\pi} \chi(n, \nu) \tilde{Y}\right) .
$$




\subsection{The large rapidity dipole density at fixed impact parameter}

At very large $\tilde{Y}$, the term corresponding to $n=0$ dominates the exponential term in the expansion (2.23) because of the expression of $\chi(n, \nu)$ (see Eq. (2.18)), and we will restrict ourselves to this case in this subsection.

We first give a useful representation for $E_{q}^{0 \nu *}$ (a general representation for $E_{q}^{n \nu *}$ can also be obtained [32]). From Eqs. (2.13) and (2.21), we get

$$
\begin{aligned}
E_{q}^{0, \nu}(\rho) & =\frac{2 \pi^{2}}{b_{0, \nu}} \rho^{2 i \nu} \int \frac{d^{2} \underline{R} e^{i \underline{q} \cdot \underline{R}}}{\{|\underline{R}-\underline{\rho} / 2||\underline{R}+\underline{\rho} / 2|\}^{1+2 i \nu}} \\
& =\frac{2 \pi^{2}}{b_{0, \nu}} \rho^{2 i \nu} e^{i \underline{q} \cdot \underline{\rho} / 2} \int \frac{d^{2} \underline{R}^{\prime} e^{i \underline{q} \cdot \underline{R^{\prime}}}}{\left\{\left|\underline{R}^{\prime}\right|\left|\underline{R}^{\prime}+\underline{\rho}\right|\right\}^{1+2 i \nu}} .
\end{aligned}
$$

Introducing the Feynman representation of the integrand

$$
\frac{1}{\left\{\left|\underline{R^{\prime}}\right|^{2}\left|\underline{R^{\prime}}+\underline{\rho}\right|^{2}\right\}^{\frac{1}{2}+i \nu}}=\frac{\Gamma(1+2 i \nu)}{\Gamma^{2}\left(\frac{1}{2}+i \nu\right)} \int_{0}^{1} \frac{[\alpha(1-\alpha)]^{i \nu-\frac{1}{2}} d \alpha}{D_{\alpha}^{1+2 i \nu}}
$$

where

$$
D_{\alpha}=(1-\alpha) \underline{R}^{\prime 2}+\alpha\left(\underline{R}^{\prime}+\underline{\rho}\right)^{2}=\left|\underline{R}^{\prime}+\alpha \underline{\rho}\right|^{2}+\underline{\rho}^{2} \alpha(1-\alpha),
$$

and setting $\underline{R}=\underline{R}^{\prime}+\alpha \underline{\rho}$, Eq. (2.24) now reads

$$
E_{q}^{0, \nu}(\rho)=\frac{2 \pi^{2}}{b_{0, \nu}} \rho^{2 i \nu} \frac{\Gamma(1+2 i \nu)}{\Gamma^{2}\left(\frac{1}{2}+i \nu\right)} \int_{0}^{1} d \alpha[\alpha(1-\alpha)]^{i \nu-\frac{1}{2}} e^{i \underline{q} \cdot\{\underline{\rho} / 2-\alpha \underline{\rho}\}} \int \frac{d^{2} \underline{R} e^{i \underline{q} \cdot \underline{R}}}{\left[\underline{R}^{2}+\underline{\rho}^{2} \alpha(1-\alpha)\right]^{1+2 i \nu}} .
$$

The integration with respect to the angle $(\underline{q}, \underline{R})$ leads to a Bessel function $J_{0}(q R)$. The integration with respect to $R=|\underline{R}|$ can then be carried out, using the formula (6.565) of Ref. 33

$$
\int \frac{J_{\nu}(q x) x^{\nu+1}}{\left[x^{2}+a^{2}\right]^{\mu+1}} d x=\frac{a^{\nu-\mu} q^{\mu} K_{\nu-\mu}(a q)}{2^{\mu} \Gamma(\mu+1)}
$$

This finally yields

$$
E_{q}^{0, \nu}(\rho)=\frac{4 \pi^{3}}{b_{0, \nu}} e^{i \underline{q} \cdot \underline{\rho} / 2}\left(\frac{q}{2}\right)^{2 i \nu} \frac{1}{\Gamma^{2}\left(\frac{1}{2}+i \nu\right)} \int_{0}^{1} d \alpha[\alpha(1-\alpha)]^{-\frac{1}{2}} e^{-i \alpha \underline{q} \cdot \underline{\rho}} K_{-2 i \nu}(q \rho \sqrt{\alpha(1-\alpha)}) .
$$

This last integration can be performed and yields (see appendix A.6)

$$
E_{q}^{0, \nu}(\rho)=\left(\frac{q}{2}\right)^{2 i \nu} 2^{-2 i \nu} \Gamma^{2}(1-i \nu)\left[J_{i \nu}\left(\frac{\rho q}{4} e^{i \Psi}\right) J_{i \nu}\left(\frac{\rho q}{4} e^{-i \Psi}\right)-J_{-i \nu}\left(\frac{\rho q}{4} e^{i \Psi}\right) J_{-i \nu}\left(\frac{\rho q}{4} e^{-i \Psi}\right)\right]
$$

where $\Psi$ is the angle $(\underline{q}, \underline{\rho})$. We will not need this explicit form in the following calculation.

Let us now compute the contribution to the density of dipole (2.23) corresponding to $n=0$. Since the longitudinal and the transverse degrees of freedom can be easily separated 
(see formula (2.23)), we will forget the exponential term depending on the rapidity $\tilde{Y}$, and will restore this dependence at the very end. We thus define $n_{\{n, \nu\}}\left(x, x^{\prime}, b\right)$ such that

$$
n\left(\underline{x}, \underline{x}^{\prime}, \tilde{Y}, \underline{b}\right)=\sum_{n=-\infty}^{+\infty} \int \frac{d \nu}{2 \pi} n_{\{n, \nu\}}\left(x, x^{\prime}, b\right) \exp \left(\frac{2 \alpha N_{c}}{\pi} \chi(n, \nu) \tilde{Y}\right) .
$$

In the asymptotic case we are interested in,

$$
n\left(\underline{x}, \underline{x}^{\prime}, \tilde{Y}, \underline{b}\right) \sim \int \frac{d \nu}{2 \pi} n_{\{0, \nu\}}\left(x, x^{\prime}, b\right) \exp \left(\frac{2 \alpha N_{c}}{\pi} \chi(0, \nu) \tilde{Y}\right) .
$$

We can now express the distribution of dipole, taking into account Eq. (2.32). When $\tilde{Y} \rightarrow \infty$, one can use a saddle approximation for the $\nu$-integration. The function $\chi(0, \nu)$ is maximum at $\nu=0$, which thus defines the saddle-point. We will thus expand $n_{\{0, \nu\}}$ around $\nu=0$. Using the expression (2.29), $n_{\{0, \nu\}}$ then reads

$$
\begin{gathered}
n_{\{0, \nu\}}\left(x, x^{\prime}, b\right)=\frac{16 \pi^{6}}{\left|b_{0, \nu}\right|^{2}} \frac{x}{x^{\prime}} \frac{1}{\Gamma^{2}\left(\frac{1}{2}+i \nu\right) \Gamma^{2}\left(\frac{1}{2}-i \nu\right)} \int \frac{d^{2} \underline{q}}{(2 \pi)^{2}} \int_{0}^{1} d \alpha \int_{0}^{1} d \beta[\alpha(1-\alpha)]^{-\frac{1}{2}}[\beta(1-\beta)]^{-\frac{1}{2}} \\
\times e^{i \underline{q} \cdot \frac{\underline{x}}{2}(1-2 \alpha)} e^{-i \underline{q} \cdot \frac{\underline{x}^{\prime}}{2}(1-2 \beta)} e^{-i \underline{q} \cdot \underline{b}} K_{2 i \nu}(q x \sqrt{\alpha(1-\alpha)}) K_{-2 i \nu}\left(q x^{\prime} \sqrt{\beta(1-\beta)}\right)
\end{gathered}
$$

Setting $\underline{v}=\frac{\underline{x}}{2}(1-2 \alpha)-\frac{\underline{x}^{\prime}}{2}(1-2 \beta)+\underline{b}$, the integration with respect to the angle $(\underline{q}, \underline{v})$ gives a Bessel function $J_{0}(q v)$. The integration with respect to $q$ can be carried out using the following formula 34

$$
\begin{aligned}
\int_{0}^{\infty} x^{\mu+1} K_{\lambda}\left(a_{1} x\right) K_{\lambda}\left(a_{2} x\right) J_{\mu}\left(a_{3} x\right) d x & \\
= & \frac{1}{2} \sqrt{\frac{\pi}{2}}\left(\frac{a_{3}}{a_{1} a_{2}}\right)^{\mu+1} \mathcal{P}_{\lambda-\frac{1}{2}}^{-\mu-\frac{1}{2}}(z)\left(z^{2}-1\right)^{-\frac{\mu}{2}-\frac{1}{4}} \Gamma(\mu+\lambda+1) \Gamma(\mu-\lambda+1),
\end{aligned}
$$

where $z=\frac{1}{2} \frac{a_{1}^{2}+a_{2}^{2}+a_{3}^{2}}{a_{1} a_{2}}$, and $\mathcal{P}$ is a Legendre function. Here $\mu=0, \lambda=2 i \nu$ and the corresponding Legendre function is

$$
\mathcal{P}_{\gamma}^{-\frac{1}{2}}(z)=(2 \pi)^{-\frac{1}{2}}\left(\frac{1}{2}+\gamma\right)^{-1}\left(z^{2}-1\right)^{-\frac{1}{4}}\left\{\left[z+\left(z^{2}-1\right)^{\frac{1}{2}}\right]^{\gamma+\frac{1}{2}}-\left[z+\left(z^{2}-1\right)^{\frac{1}{2}}\right]^{\gamma+\frac{1}{2}}\right\} .
$$

In the case we are interested in, $a_{1}=x \sqrt{\alpha(1-\alpha)}, a_{2}=x^{\prime} \sqrt{\beta(1-\beta)}$ and $a_{3}=b$. In the domain $b \gg x, x^{\prime}$ (where the total cross-section gets its dominant contribution: see subsection 2.2),

$$
z \simeq \frac{b^{2}}{2 x x^{\prime}}[\alpha(1-\alpha) \beta(1-\beta)]^{-\frac{1}{2}} \gg 1
$$

and thus

$$
\begin{aligned}
\int q & K_{2 i \nu}(q x \sqrt{\alpha(1-\alpha)}) K_{-2 i \nu}\left(q x^{\prime} \sqrt{\beta(1-\beta)}\right) J_{0}(q v) d q \simeq \frac{1}{2} \frac{1}{2 i \nu} \Gamma(1+2 i \nu) \Gamma(1-2 i \nu) \\
& \times \frac{1}{b^{2}}\left\{\left[\frac{b^{2}}{x x^{\prime}}\right]^{2 i \nu}[\alpha(1-\alpha) \beta(1-\beta)]^{-i \nu}-\left[\frac{b^{2}}{x x^{\prime}}\right]^{-2 i \nu}[\alpha(1-\alpha) \beta(1-\beta)]^{i \nu}\right\} .
\end{aligned}
$$


Using Ref. 29,

$$
a_{n, \nu}=\frac{\pi^{4} / 2}{\nu^{2}+n^{2} / 4}=\frac{\left|b_{n, \nu}\right|^{2}}{2 \pi^{2}}
$$

which gives for $n_{\{0, \nu\}}$

$$
\begin{aligned}
& n_{\{0, \nu\}}\left(\underline{x}, \underline{x^{\prime}}, \underline{b}\right) \simeq-\frac{2 i \nu}{\pi} \frac{x}{x^{\prime}} \frac{1}{b^{2}} \frac{\Gamma(1+2 i \nu) \Gamma(1-2 i \nu)}{\Gamma^{2}\left(\frac{1}{2}+i \nu\right) \Gamma^{2}\left(\frac{1}{2}-i \nu\right)} \int_{0}^{\infty} d \alpha \int_{0}^{\infty} d \beta \\
& \quad \times\left\{\left(\frac{b^{2}}{x x^{\prime}}\right)^{2 i \nu}[\alpha(1-\alpha) \beta(1-\beta)]^{-\frac{1}{2}-i \nu}-\left(\frac{b^{2}}{x x^{\prime}}\right)^{-2 i \nu}[\alpha(1-\alpha) \beta(1-\beta)]^{-\frac{1}{2}+i \nu}\right\} .
\end{aligned}
$$

The integration with respect to $\alpha$ and $\beta$ can now be carried out. This yields

$$
n_{\{0, \nu\}}\left(\underline{x}, \underline{x}^{\prime}, \underline{b}\right) \simeq-\frac{2 i \nu}{\pi} \frac{x}{x^{\prime}} \frac{1}{b^{2}}\left\{\frac{\Gamma(1+2 i \nu)}{\Gamma(1-2 i \nu)} \frac{\Gamma^{2}\left(\frac{1}{2}-i \nu\right)}{\Gamma^{2}\left(\frac{1}{2}+i \nu\right)}\left(\frac{b^{2}}{x x^{\prime}}\right)^{2 i \nu}-c . c\right\} .
$$

Using the doubling formula, one finally obtains

$$
n_{\{0, \nu\}}\left(\underline{x}, \underline{x}^{\prime}, \underline{b}\right) \simeq-\frac{2 i \nu}{\pi} \frac{x}{x^{\prime}} \frac{1}{b^{2}}\left\{\left(\frac{16 b^{2}}{x x^{\prime}}\right)^{2 i \nu}-\left(\frac{16 b^{2}}{x x^{\prime}}\right)^{-2 i \nu}+\mathcal{O}\left(\nu^{3}\right)\right\} .
$$

This can now be inserted in Eq. (2.32) to calculate the large $\tilde{Y}$ limit of the density, when $b^{2} / x x^{\prime} \ll 1$. We develop $\chi(0, \nu)$ around $\nu=0$

$$
\chi(0, \nu) \sim 2 \ln 2-7 \zeta(3) \nu^{2}+\mathcal{O}\left(\nu^{4}\right)
$$

and take into account that $\chi(0,-\nu)=\chi(0, \nu)$. One has then to compute

$$
n\left(\underline{x}, \underline{x}^{\prime}, \tilde{Y}, \underline{b}\right) \simeq-\frac{x}{x^{\prime}} \frac{1}{b^{2}} \int_{-\infty}^{+\infty} \frac{d \nu}{2 \pi} \frac{4 i \nu}{\pi} \exp \left(\frac{2 \alpha_{s} N_{c}}{\pi} 2 \ln 2 \tilde{Y}-\frac{2 \alpha_{s} N_{c}}{\pi} 7 \zeta(3) \tilde{Y} \nu^{2}+2 i \nu \ln \frac{16 b^{2}}{x x^{\prime}}\right) .
$$

After making the replacement $\nu^{\prime}=\nu-i\left(\ln \frac{16 b^{2}}{x x^{\prime}}\right) /\left(\frac{2 \alpha_{s} N_{c}}{\pi} 7 \zeta(3) \tilde{Y}\right)$, the gaussian integration with respect to $\nu^{\prime}$ finally yields

$$
n\left(\underline{x}, \underline{x^{\prime}}, \tilde{Y}, \underline{b}\right) \simeq \frac{x}{4 b^{2} x^{\prime}} \frac{\ln \left(16 b^{2} / x x^{\prime}\right)}{\left(\frac{7}{2} \alpha_{s} N_{c} \zeta(3) \tilde{Y}\right)^{3 / 2}} \exp \left\{\frac{4 \alpha_{s} N_{c}}{\pi} \ln 2 \tilde{Y}\right\} \exp \left\{-\frac{\ln ^{2}\left(16 b^{2} / x x^{\prime}\right)}{\frac{14 \alpha_{s} N_{c}}{\pi} \zeta(3) \tilde{Y}}\right\} .
$$

This result is valid in the domain

$$
\frac{2 \alpha_{s} N_{c}}{\pi} 7 \zeta(3) \tilde{Y} \ll \ln \frac{16 b^{2}}{x x^{\prime}} \ll 1
$$

This expression corrects the expression (8) of Ref. [16] when one considers the distribution in transverse space. Numerical simulations and approximate analytical calculations indeed confirm this factor 16 in the transverse distribution [35]. Note that it is only in this asymptotic regime that $n\left(\underline{x}, \underline{x}^{\prime}, \tilde{Y}, \underline{b}\right)$ has no angular dependence. 


\subsection{Exact result for the onium-onium cross-section at fixed impact parameter and equivalence with BFKL Pomeron}

Let us now compute the onium-onium scattering amplitude at fixed impact parameter according to the process displayed in figure 1 . We denote $\underline{x}_{a 1}\left(\underline{x}_{b 1}\right)$ the transverse coordinate of the heavy quark (antiquark) making up the right moving onium and $\underline{x}_{a 2}\left(\underline{x}_{b 2}\right)$ the coordinates of the corresponding quark (antiquark) making the left moving onium. These onia of transverse sizes $\underline{x}_{1}=\underline{x}_{a 1}-\underline{x}_{b 1}$ and $\underline{x}_{2}=\underline{x}_{a 2}-\underline{x}_{b 2}$ scatter through the exchange of a pair of gluons between two elementary dipoles, respectively of transverse size $\underline{x}_{1}^{\prime}$ and $\underline{x}_{2}^{\prime}$, located at $\underline{b}_{1}^{\prime}$ and $\underline{b}_{2}^{\prime}$ with respect to the reference point $\underline{0}$ (which is arbitrary due to translation invariance). These two elementary dipoles are produced by the two heavy quark-antiquark pairs at a distance $\underline{b}_{1}$ and $\underline{b}_{2}$ from their center of mass.

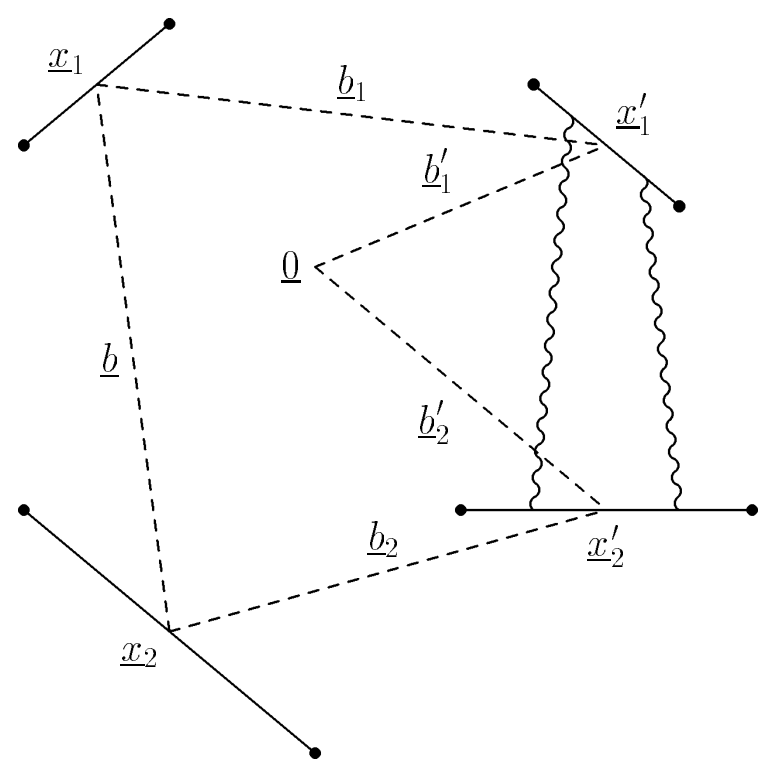

Figure 1: Onium-Onium scattering at leading order.

In order to compute $F^{(1)}$ as given by Eq. (2.7), we now use the result (2.15, 2.16) for the dipole density and the following expansion of the dipole-dipole cross-section $\sigma_{D D}$

$$
\begin{aligned}
& \sigma_{D D}\left(\underline{x}_{1}^{\prime}, \underline{x}_{2}^{\prime}, \underline{b}_{1}^{\prime}-\underline{b}_{2}^{\prime}\right)=\frac{2 \alpha_{s}^{2}}{(2 \pi)^{2}} \sum_{n=-\infty}^{+\infty} \int_{-\infty}^{+\infty} d \nu \int d^{2} \underline{w}\left(\nu^{2}+\frac{n^{2}}{4}\right) \frac{1+(-1)^{n}}{\left(\nu^{2}+\left(\frac{n-1}{2}\right)^{2}\right)\left(\nu^{2}+\left(\frac{n+1}{2}\right)^{2}\right)} \\
& \times E^{n, \nu *}\left(\underline{b}_{1}^{\prime}+\frac{\underline{x}_{1}^{\prime}}{2}-\underline{w}, \underline{b_{1}}-\frac{\underline{x}_{1}^{\prime}}{2}-\underline{w}\right) E^{n, \nu}\left(\underline{b}_{2}^{\prime}+\frac{\underline{x}_{2}^{\prime}}{2}-\underline{w}, \underline{b}_{2}^{\prime}-\frac{\underline{x}_{2}^{\prime}}{2}-\underline{w}\right)
\end{aligned}
$$

which is proved in appendix A.2. The full expression obtained is

$$
\begin{aligned}
& F^{(1)}\left(\underline{x}_{1}, \underline{x}_{2}, \tilde{Y}, \underline{b}\right)=-\frac{\alpha_{s}^{2}(16)^{2}}{(2 \pi)^{2}} \sum_{n_{1}=-\infty}^{+\infty} \sum_{n_{2}=-\infty}^{+\infty} \sum_{n=-\infty}^{+\infty} \int_{-\infty}^{\infty} \frac{d \nu_{1}}{(2 \pi)^{3}} \int_{-\infty}^{\infty} \frac{d \nu_{2}}{(2 \pi)^{3}} \int_{-\infty}^{\infty} d \nu \int \frac{d^{2} \underline{w}_{1}}{x_{1}^{\prime 2} \int \frac{d^{2} \underline{w}_{2}}{x_{2}^{\prime 2}}} \\
& \times \int d^{2} \underline{w} \int \frac{d^{2} \underline{x}_{1}^{\prime}}{2 \pi x_{1}^{\prime 2}} \int \frac{d^{2} \underline{x}_{2}^{\prime}}{2 \pi x_{2}^{\prime 2}} \int d^{2} \underline{b}_{1} \int d^{2} \underline{b}_{2} \int d^{2} \underline{b}_{i n t} \delta^{2}\left(\underline{b}_{1}-\underline{b}_{2}+\underline{b}_{i n t}-\underline{b}\right)\left(\nu_{1}^{2}+\frac{n_{1}^{2}}{4}\right)\left(\nu_{2}^{2}+\frac{n_{2}^{2}}{4}\right) \\
& \times\left(\nu^{2}+\frac{n^{2}}{4}\right) \frac{1+(-1)^{n}}{\left(\nu^{2}+\left(\frac{n-1}{2}\right)^{2}\right)\left(\nu^{2}+\left(\frac{n+1}{2}\right)^{2}\right)} \exp \left(\frac{2 \alpha_{s} N_{c}}{\pi}\left(\chi\left(n_{1}, \nu_{1}\right) \tilde{Y}_{1}+\chi\left(n_{2}, \nu_{2}\right) \tilde{Y}_{2}\right)\right)
\end{aligned}
$$




$$
\begin{aligned}
& \times E^{n_{1}, \nu_{1}}\left(\underline{b}_{1}+\frac{\underline{x}_{1}^{\prime}}{2}-\underline{w}_{1}, \underline{b}_{1}-\frac{\underline{x}_{1}^{\prime}}{2}-\underline{w}_{1}\right) E^{n_{1}, \nu_{1} *}\left(\frac{\underline{x}_{1}}{2}-\underline{w}_{1},-\frac{\underline{x}_{1}}{2}-\underline{w}_{1}\right) \\
& \times E^{n_{2}, \nu_{2} *}\left(\underline{b}_{2}+\frac{\underline{x}_{2}^{\prime}}{2}-\underline{w}_{2}, \underline{b}_{2}-\frac{\underline{x}_{2}^{\prime}}{2}-\underline{w}_{2}\right) E^{n_{2}, \nu_{2}}\left(\frac{\underline{x}_{2}}{2}-\underline{w}_{2},-\frac{\underline{x}_{2}}{2}-\underline{w}_{2}\right) \\
& \times E^{n, \nu *}\left(\underline{b}_{1}+\frac{\underline{x}_{1}^{\prime}}{2}-\underline{w}, \underline{b}_{1}-\frac{\underline{x}_{1}^{\prime}}{2}-\underline{w}\right) E^{n, \nu}\left(\underline{b}_{i n t}+\underline{b}_{1}+\frac{\underline{x}_{2}^{\prime}}{2}-\underline{w}_{1}, \underline{b}_{i n t}+\underline{b}_{1}-\frac{\underline{x}_{2}^{\prime}}{2}-\underline{w}_{1}\right)
\end{aligned}
$$

where we have used the fact that $\sigma_{D D}\left(\underline{x}_{1}^{\prime}, \underline{x}_{2}^{\prime}, \underline{b}_{1}^{\prime}-\underline{b}_{2}^{\prime}\right)$ is translationally invariant and only depends on $\underline{b}_{i n t}=\underline{b}_{2}^{\prime}-\underline{b}_{1}^{\prime}$. The quantum numbers $n_{1}, \nu_{1}$ and $n_{2}, \nu_{2}$ correspond respectively to the dipole distributions $n\left(\underline{x}_{1}, \underline{x}_{1}^{\prime}, \tilde{Y}_{1}, \underline{b}_{1}\right)$ and $n\left(\underline{x}_{2}, \underline{x}_{2}^{\prime}, \tilde{Y}_{2}, \underline{b}_{2}\right)$.

Note the existence of a term $1+(-1)^{n}$ which arises from the $s \leftrightarrow u$ symetry of the elementary dipole-dipole cross-section since the gluon is a massless vector boson (see appendix A.2). As a consequence only the even $n$ will contribute to the scattering amplitude.

The integration with respect to $\underline{b}_{2}$ can be performed through the delta distribution. Then, the remaining expression can be strongly simplified when using the following orthonormalization condition for the functions $E^{n, \nu}$ (see Eq. (A.16) of Ref. [29])

$$
\begin{gathered}
\int \frac{d^{2} \underline{\rho_{1}} d^{2} \underline{\rho_{2}}}{\left|\rho_{12}\right|^{4}} E^{n, \nu}\left(\rho_{10}, \rho_{20}\right) E^{m, \mu *}\left(\rho_{10^{\prime}}, \rho_{20^{\prime}}\right)=a_{n, \nu} \delta_{n, m} \delta(\nu-\mu) \delta^{2}\left(\rho_{00^{\prime}}\right) \\
+(-1)^{n} b_{n, \nu}\left|\rho_{00^{\prime}}\right|^{-2-4 i \nu}\left(\rho_{00^{\prime}} / \rho_{00^{\prime}}^{*}\right)^{n} \delta_{n,-m} \delta(\nu+\mu) .
\end{gathered}
$$

Note that this equation corrects Eq. (A.16) of Ref. [29] since the factor $(-1)^{n}$ in the second term of the right-hand side was missing. This term arises from the fact that

$$
\left(\frac{z^{2}-1}{z^{2 *}-1}\right)^{\frac{n}{2}}=(-1)^{n}\left(\frac{1-z^{2}}{1-z^{2 *}}\right)^{\frac{n}{2}},
$$

which has to be taken into account when performing the last transformation in Eq. (A.19) of Ref. [29]. Applying the relation (2.48) for $\underline{\rho}_{1}=\underline{b}_{1}+\frac{\underline{x}_{1}^{\prime}}{2}, \quad \underline{\rho}_{2}=\underline{b}_{1}-\frac{\underline{x}_{1}^{\prime}}{2}, \quad \underline{\rho}_{0}=\underline{w}_{1}$ and $\underline{\rho}_{0^{\prime}}=\underline{w}$ on one hand, for $\underline{\rho}_{1}^{\prime}=\underline{b}_{1}+\underline{b}_{i n t}+\frac{\underline{x}_{2}^{\prime}}{2}, \quad \underline{\rho}_{2}^{\prime}=\underline{b}_{1}+\underline{b}_{i n t}-\frac{\underline{x}_{2}^{\prime}}{2}, \quad \underline{\rho}_{0}=\underline{w}_{2}+\underline{b}$ and $\underline{\rho}_{0^{\prime}}=\underline{w}$ on the other hand, and using the equality $d^{2} \underline{b}_{1} d^{2} \underline{b}_{i n t} d^{2} \underline{x}_{1}^{\prime} d^{2} \underline{x}_{2}^{\prime}=d^{2} \underline{\rho}_{1} d^{2} \underline{\rho}_{2} d^{2} \underline{\rho}_{1}^{\prime} d^{2} \underline{\rho}_{2}^{\prime}$, the integration over these transverse variables gives

$$
\begin{aligned}
& F^{(1)}\left(\underline{x}_{1}, \underline{x}_{2}, \tilde{Y}, \underline{b}\right)=-\frac{\alpha_{s}^{2}}{(2 \pi)^{2}} \frac{(16)^{2}}{(2 \pi)^{2}} \sum_{n_{1}=-\infty}^{+\infty} \sum_{n_{2}=-\infty}^{+\infty} \sum_{n=-\infty}^{+\infty} \int_{-\infty}^{\infty} \frac{d \nu_{1}}{(2 \pi)^{3}} \int_{-\infty}^{\infty} \frac{d \nu_{2}}{(2 \pi)^{3}} \int_{-\infty}^{\infty} d \nu \int d^{2} \underline{w}_{1} \int d^{2} \underline{w}_{2} \\
& \times \int d^{2} \underline{w}\left(\nu_{1}^{2}+\frac{n_{1}^{2}}{4}\right)\left(\nu_{2}^{2}+\frac{n_{2}^{2}}{4}\right)\left(\nu^{2}+\frac{n^{2}}{4}\right) \frac{1+(-1)^{n}}{\left(\nu^{2}+\left(\frac{n-1}{2}\right)^{2}\right)\left(\nu^{2}+\left(\frac{n+1}{2}\right)^{2}\right)} \\
& \times \exp \left(\frac{2 \alpha_{s} N_{c}}{\pi}\left(\chi\left(n_{1}, \nu_{1}\right) \tilde{Y}_{1}+\chi\left(n_{2}, \nu_{2}\right) \tilde{Y}_{2}\right)\right) \\
& \times\left[a_{n_{1}, \nu_{1}} \delta_{n_{1}, n} \delta\left(\nu_{1}-\nu\right) \delta^{2}\left(\underline{w}-\underline{w}_{1}\right)+b_{n_{1}, \nu_{1}}\left|\underline{w}-\underline{w}_{1}\right|^{-2-4 i \nu_{1}}\left(\frac{w-w_{1}}{w^{*}-w_{1}^{*}}\right)^{n_{1}} \delta_{n_{1},-n} \delta\left(\nu_{1}+\nu\right)(-1)^{n_{1}}\right] \\
& \times\left[a_{n_{2}, \nu_{2}} \delta_{n_{2}, n} \delta\left(\nu_{2}-\nu\right) \delta^{2}\left(\underline{w}-\underline{w}_{2}-\underline{b}\right)+b_{n, \nu} \mid \underline{w}-\underline{w}_{2}-\underline{b}^{-2-4 i \nu}\left(\frac{w-w_{2}-b}{w^{*}-w_{2}^{*}-b^{*}}\right)^{n} \delta_{n,-n_{2}}\right. \\
& \left.\times \delta\left(\nu_{2}+\nu\right)(-1)^{n}\right] E^{n_{1}, \nu_{1} *}\left(\frac{\underline{x}_{1}}{2}-\underline{w}_{1},-\frac{\underline{x}_{1}}{2}-\underline{w}_{1}\right) E^{n_{2}, \nu_{2}}\left(\frac{\underline{x}_{2}}{2}-\underline{w}_{2},-\frac{\underline{x}_{2}}{2}-\underline{w}_{2}\right) .
\end{aligned}
$$


The two terms involving the normalisation factors $b_{n_{1}, \nu_{1}}$ and $b_{n, \nu}$ can be reexpressed using the fact that $E^{n, \nu}$ and $E^{n, \nu *}$ are related by the following relation, which corrects Eq. (A.12) of Ref. [29] (see appendix A.7)

$$
E^{n, \nu *}\left(\underline{\rho}_{10}, \underline{\rho}_{20}\right)=\frac{b_{n, \nu}^{*}}{a_{n, \nu}} \int d^{2} \rho_{0^{\prime}} E^{n, \nu}\left(\underline{\rho}_{10^{\prime}}, \underline{\rho}_{20^{\prime}}\right)\left|\rho_{00^{\prime}}\right|^{-2+4 i \nu}\left(\frac{\rho_{0^{\prime} 0}^{*}}{\rho_{0^{\prime} 0}}\right)^{n}(-1)^{n} .
$$

Note that this relation arises from the equivalence between the two corresponding representations of $S L(2, C)$ [31]. The integration with respect to $\underline{w}_{1}$ and $\underline{w}_{2}$ can then be performed. It gives

$$
\begin{gathered}
\int d^{2} \underline{w}_{1} E^{n, \nu}\left(\frac{\underline{x}_{1}}{2}-\underline{w}_{1},-\frac{\underline{x}_{1}}{2}-\underline{w}_{1}\right)\left|\underline{w}-\underline{w}_{1}\right|^{-2+4 i \nu}\left(\frac{w-w_{1}}{w^{*}-w_{1}^{*}}\right)^{-n}(-1)^{-n} \\
=\frac{a_{n, \nu}}{b_{n, \nu}^{*}} E^{n, \nu *}\left(\frac{\underline{x}_{1}}{2}-\underline{w},-\frac{\underline{x}_{1}}{2}-\underline{w}\right) .
\end{gathered}
$$

and

$$
\begin{gathered}
\int d^{2} \underline{w}_{2} E^{-n,-\nu}\left(\frac{\underline{x}_{2}}{2}-\underline{w}_{2},-\frac{\underline{x}_{2}}{2}-\underline{w}_{2}\right)\left|\underline{w}-\underline{w}_{2}-\underline{b}\right|^{-2-4 i \nu}\left(\frac{w-w_{2}-b}{w^{*}-w_{2}^{*}-b^{*}}\right)^{n}(-1)^{n} \\
=\frac{a_{-n,-\nu}}{b_{-n,-\nu}^{*}} E^{-n,-\nu *}\left(\frac{\underline{x}_{2}}{2}-\underline{w}+\underline{b},-\frac{\underline{x}_{2}}{2}-\underline{w}+\underline{b}\right),
\end{gathered}
$$

where we have used the fact that $E^{-n,-\nu *}\left(\rho_{10}, \rho_{20}\right)=E^{n, \nu}\left(\rho_{10}, \rho_{20}\right)$. Since $b_{-n,-\nu *}=b_{n, \nu}$ (see Eq. (2.22) ) and $a_{-n,-\nu}=a_{n, \nu}$ (see Eq. (2.38)), the contributions of the four terms obtained after expanding the brackets in Eq. (2.50) are identical and one finally gets, using $\tilde{Y}=Y_{1}+\tilde{Y}_{2}$,

$$
\begin{aligned}
& F^{(1)}\left(x_{1}, x_{2}, \tilde{Y}, b\right)=-\frac{\alpha_{s}^{2}}{(2 \pi)^{2}}(2 \pi)^{6} \sum_{n=-\infty}^{+\infty} \int_{-\infty}^{\infty} d \nu \int d^{2} \underline{w}\left(\nu^{2}+\frac{n^{2}}{4}\right) \frac{1+(-1)^{n}}{\left(\nu^{2}+\left(\frac{n-1}{2}\right)^{2}\right)\left(\nu^{2}+\left(\frac{n+1}{2}\right)^{2}\right)} \\
& \times \exp \left(\frac{2 \alpha_{s} N_{c}}{\pi} \chi(n, \nu) \tilde{Y}\right) E^{n, \nu *}\left(\frac{\underline{x}_{1}}{2}-\underline{w},-\frac{\underline{x}_{1}}{2}-\underline{w}\right) E^{n, \nu}\left(\frac{\underline{x}_{2}}{2}-\underline{w}+\underline{b},-\frac{\underline{x}_{2}}{2}-\underline{w}+\underline{b}\right) .
\end{aligned}
$$

Some comments are in order about this result, obtained without any approximation. First, it is clearly independent of the choice of the reference frame, since the result only depends on the total rapidity $\tilde{Y}$. In appendix A.4 we explicitly show that this formula describes in an equivalent way, in the laboratory frame of the left-moving onium (which is defined to be the frame where this onium is moving relativistically, but has not enough rapidity to reveal its soft gluon contents), the scattering of this non evolved heavy quark-antiquark pair off one excited dipole at a distance $b$ from the center of mass of the fast right-moving onium.

Second, and more importantly, it explicitly proves the exact equivalence between the dipole and the BFKL approaches at leading order. Indeed, taking into account form factors when coupling the $t$-channel bound state of reggeized gluons to the external quark-antiquark pairs (see appendix A.2 for details) and the difference of definition of amplitudes $\left(A_{\text {dipole }}=\right.$ $\left.\frac{1}{2 s} A_{B F K L}\right)$, we should have the following relation between the dipole and the BFKL result

$$
F^{(1)}\left(\underline{x}_{1}, \underline{x}_{2}, \tilde{Y}, \underline{b}\right)=\alpha_{s}^{2} \int \frac{d \omega}{2 \pi i} \exp (\omega \tilde{Y})\left[f_{\omega}\left(\underline{x}_{a 1}, \underline{x}_{b 1}, \underline{x}_{a 2}, \underline{x}_{b 2}\right)+f_{\omega}\left(\underline{x}_{b 1}, \underline{x}_{a 1}, \underline{x}_{a 2}, \underline{x}_{b 2}\right)\right]
$$


where $f_{\omega}\left(\underline{x}_{a 1}, \underline{x}_{b 1}, \underline{x}_{a 2}, \underline{x}_{b 2}\right)$ is defined by equation (26) of Ref. 29]

$$
\begin{aligned}
& f_{\omega}\left(\underline{x}_{a 1}, \underline{x}_{b 1}, \underline{x}_{a 2}, \underline{x}_{b 2}\right)=\sum_{n=-\infty}^{+\infty} \int_{-\infty}^{+\infty} \int d^{2} \underline{x}_{0}\left(\nu^{2}+\frac{n^{2}}{4}\right) \frac{1}{\left(\nu^{2}+\left(\frac{n-1}{2}\right)^{2}\right)\left(\nu^{2}+\left(\frac{n+1}{2}\right)^{2}\right)} \\
& \times \frac{1}{\omega-\frac{2 \alpha_{s} N_{c}}{\pi} \chi(n, \nu)} E^{n, \nu *}\left(\underline{x}_{a 2}-\underline{x}_{0}, \underline{x}_{b 2}-\underline{x}_{0}\right) E^{n, \nu}\left(\underline{x}_{a 1}-\underline{x}_{0}, \underline{x}_{b 1}-\underline{x}_{0}\right) .
\end{aligned}
$$

In this formula the integrand gets a factor $(-1)^{n}$ when permuting $x_{a 1} \leftrightarrow x_{b 1}$. Performing the changes of variable $\underline{w}=\underline{x}_{0}-\frac{\underline{x}_{a 1}+\underline{x}_{b 1}}{2}$ and $(n, \nu) \rightarrow(-n,-\nu)$, one then recovers exactly the expansion (2.54) with the factor $1+(-1)^{n}$, which proves the result. Note that the equivalence between the BFKL and dipole kernel can be also proven by comparing the real and virtual graphs in covariant and light-cone quantization. The result is that the sum of real and virtual contributions is identical in both case, although each of these terms differs. Thus, this result is true only for inclusive quantities [17].

Defining $F_{\{n, \nu\}}^{(1)}$ as

$$
F^{(1)}\left(\underline{x}_{1}, \underline{x}_{2}, \tilde{Y}, \underline{b}\right)=\sum_{n=-\infty}^{+\infty} \int_{-\infty}^{+\infty} \frac{d \nu}{2 \pi} F_{\{n, \nu\}}^{(1)}\left(\underline{x}_{1}, \underline{x}_{2}, \underline{b}\right) \exp \left(\frac{2 \alpha_{s} N_{c}}{\pi} \chi(n, \nu) \tilde{Y}\right)
$$

and using Eqs. (2.19) and (2.31), equation (2.54) can be rewritten as

$$
F_{\{n, \nu\}}^{(1)}\left(\underline{x}_{1}, \underline{x}_{2}, \underline{b}\right)=-\frac{\pi \alpha_{s}^{2} x_{2}^{2}}{8} \frac{1+(-1)^{n}}{\left(\nu^{2}+\left(\frac{n-1}{2}\right)^{2}\right)\left(\nu^{2}+\left(\frac{n+1}{2}\right)^{2}\right)} n_{\{n, \nu\}}\left(\underline{x}_{1}, \underline{x}_{2}, \underline{b}\right) .
$$

In the asymptotic regime where one can keep only the term corresponding to $n=0$, this relation simplifies to

$$
F_{\{0, \nu\}}^{(1)}\left(\underline{x}_{1}, \underline{x}_{2}, \underline{b}\right)=-\frac{\pi \alpha_{s}^{2} x_{2}^{2}}{4} \frac{1}{\left(\nu^{2}+\frac{1}{4}\right)^{2}} n_{\{0, \nu\}}\left(\underline{x}_{1}, \underline{x}_{2}, \underline{b}\right) .
$$

Computing $F^{(1)}\left(\underline{x}_{1}, \underline{x}_{2}, \tilde{Y}, \underline{b}\right)$ by a saddle point method as we $\operatorname{did}$ for $n\left(\underline{x}_{1}, \underline{x}_{2}, \tilde{Y}, \underline{b}\right)$ (see Eq. (2.43)), one has to expand the prefactor around $\nu=0$, which yields

$$
\begin{aligned}
F^{(1)}\left(\underline{x}_{1}, \underline{x}_{2}, \tilde{Y}, \underline{b}\right) & \simeq-4 \pi \alpha_{s}^{2} x_{2}^{2} n\left(\underline{x}_{1}, \underline{x}_{2}, \tilde{Y}, \underline{b}\right) \\
& \simeq-\pi \alpha_{s}^{2} \frac{x_{1} x_{2}}{b^{2}} \frac{\ln \left(16 b^{2} / x_{1} x_{2}\right)}{\left(\frac{7}{2} \alpha_{s} N_{c} \zeta(3) \tilde{Y}\right)^{3 / 2}} \exp \left\{\frac{4 \alpha_{s} N_{c}}{\pi} \ln 2 \tilde{Y}\right\} \exp \left\{-\frac{\ln ^{2}\left(16 b^{2} / x_{1} x_{2}\right)}{\frac{14 \alpha_{s} N_{c}}{\pi} \zeta(3) \tilde{Y}}\right\}
\end{aligned}
$$

in the domain

$$
\frac{2 \alpha_{s} N_{c}}{\pi} 7 \zeta(3) \tilde{Y} \ll \ln \frac{16 b^{2}}{x_{1} x_{2}} \ll 1 .
$$

This result, which differs from Eq. (10) of Ref. [16 by a factor 16, is in aggreement with numerical simulations [35]. 


\subsection{Calculation of the onium-onium total cross-section}

In this subsection we compute the onium-onium total cross-section. It is related to the oniumonium cross-section at fixed impact parameter by

$$
F_{\text {tot }}^{(1)}\left(\underline{x}_{1}, \underline{x}_{2}, \tilde{Y}\right)=\int d^{2} \underline{b} F^{(1)}\left(\underline{x}_{1}, \underline{x}_{2}, \tilde{Y}, \underline{b}\right) .
$$

We show that, provided the elementary dipole-dipole cross section is integrated over distances but not averaged over angles, one can get the onium-onium total cross-section by a simple direct calculation.

Combining formulae (2.7) and (2.62), one obtains

$$
\begin{aligned}
F_{t o t}^{(1)}\left(\underline{x}_{1}, \underline{x}_{2}, \tilde{Y}\right)= & -\frac{1}{2} \int \frac{d^{2} \underline{x}_{1}^{\prime}}{2 \pi x_{1}^{\prime 2}} \frac{d^{2} \underline{x}_{2}^{\prime}}{2 \pi x_{2}^{\prime 2}} d^{2} \underline{b}_{1} d^{2} \underline{b}_{2} d^{2}\left(\underline{b}_{2}^{\prime}-\underline{b}_{1}^{\prime}\right) \\
& \times n\left(\underline{x}_{1}, \underline{x}_{1}^{\prime}, \underline{b}_{1}, \tilde{Y}_{1}\right) n\left(\underline{x}_{2}, \underline{x}_{2}^{\prime}, \underline{b}_{2}, \tilde{Y}_{2}\right) \sigma_{D D}\left(\underline{x}_{1}^{\prime}, \underline{x}_{2}^{\prime}, \underline{b}_{1}^{\prime}-\underline{b}_{2}^{\prime}\right) \\
= & -\frac{1}{2} \int \frac{d^{2} \underline{x}_{1}^{\prime}}{2 \pi x_{1}^{\prime 2}} \frac{d^{2} \underline{x}_{2}^{\prime}}{2 \pi x_{2}^{\prime 2}} n\left(\underline{x}_{1}, \underline{x}_{1}^{\prime}, \tilde{Y}_{1}\right) n\left(\underline{x}_{2}, \underline{x}_{2}^{\prime}, \tilde{Y}_{2}\right) \int d^{2}\left(\underline{b}_{2}^{\prime}-\underline{b}_{1}^{\prime}\right) \sigma_{D D}\left(\underline{x}_{1}^{\prime}, \underline{x}_{2}^{\prime}, \underline{b}_{1}^{\prime}-\underline{b}_{2}^{\prime}\right),
\end{aligned}
$$

where $n\left(\underline{x}_{i}, \underline{x}_{i}^{\prime}, \tilde{Y}_{i}\right)$ is the integrated density of dipoles

$$
n\left(\underline{x}, \underline{x}^{\prime}, \tilde{Y}\right)=\int d^{2} \underline{b} n\left(\underline{x}, \underline{x}^{\prime}, \tilde{Y}, \underline{b}\right) .
$$

Thus, since $\underline{b}$ and $\underline{q}$ are Fourier conjugated,

$$
\begin{aligned}
n\left(\underline{x}, \underline{x}^{\prime}, \tilde{Y}\right) & =\sum_{n=-\infty}^{+\infty} \int \frac{d \nu}{2 \pi} \lim _{q \rightarrow 0} E_{q}^{n, \nu *}(x) E_{q}^{n, \nu}\left(x^{\prime}\right) \frac{|x|}{\left|x^{\prime}\right|} \exp \left(\frac{2 \alpha N_{c}}{\pi} \chi(n, \nu) \tilde{Y}\right) \\
& =\sum_{n=-\infty}^{+\infty} \int \frac{d \nu}{2 \pi} \frac{|x|}{\left|x^{\prime}\right|}\left(\frac{x^{*} x^{\prime}}{x x^{\prime *}}\right)^{n / 2}\left|\frac{x^{\prime}}{x}\right|^{-2 i \nu} \exp \left(\frac{2 \alpha N_{c}}{\pi} \chi(n, \nu) \tilde{Y}\right)
\end{aligned}
$$

where we have used Eq. (2.23) and the expansion (A.32) of $E_{q}^{n, \nu}$ for $q \rightarrow 0$. The integration of $\sigma_{D D}\left(\underline{x}_{1}^{\prime}, \underline{x}_{2}^{\prime}, \underline{b}_{1}^{\prime}-\underline{b}_{2}^{\prime}\right)$ with respect to the distance $\underline{b}_{2}^{\prime}-\underline{b}_{1}^{\prime}$ is performed in appendix A.2 and is given by formula (A.33), which still depends on the orientation of the elementary dipoles. One now gets for $F_{t o t}^{(1)}$

$$
\begin{aligned}
& F_{\text {tot }}^{(1)}\left(\underline{x}_{1}, \underline{x}_{2}, \tilde{Y}\right)=-\frac{1}{2} \sum_{n=-\infty}^{+\infty} \int_{-\infty}^{+\infty} d \nu \sum_{n_{1}=-\infty}^{+\infty} \int_{-\infty}^{+\infty} \frac{d \nu_{1}}{2 \pi} \sum_{n_{2}=-\infty}^{+\infty} \int_{-\infty}^{+\infty} \frac{d \nu_{2}}{2 \pi} \int \frac{d^{2} \underline{x}_{1}^{\prime}}{2 \pi x_{1}^{\prime 2}} \frac{d^{2} \underline{x}_{2}^{\prime}}{2 \pi x_{2}^{\prime 2}} \\
& \times \frac{x_{1}}{x_{1}^{\prime}}\left(\frac{x_{1}^{*} x_{1}^{\prime}}{x_{1} x_{1}^{\prime *}}\right)^{n_{1} / 2}\left|\frac{x_{1}^{\prime}}{x_{1}}\right|^{-2 i \nu_{1}} \frac{x_{2}}{x_{2}^{\prime}}\left(\frac{x_{2}^{*} x_{2}^{\prime}}{x_{2} x_{2}^{\prime *}}\right)^{n_{2} / 2}\left|\frac{x_{2}^{\prime}}{x_{2}}\right|^{-2 i \nu_{2}} \exp \left(\frac{2 \alpha N_{c}}{\pi}\left(\chi\left(n_{1}, \nu_{1}\right) \tilde{Y}_{1}+\chi\left(n_{2}, \nu_{2}\right) \tilde{Y}_{2}\right)\right) \\
& \times \alpha_{s}^{2} \frac{x_{1}^{\prime} x_{2}^{\prime}}{4} \frac{1+(-1)^{n}}{\left(\nu^{2}+\left(\frac{n-1}{2}\right)^{2}\right)\left(\nu^{2}+\left(\frac{n-1}{2}\right)^{2}\right)}\left(\frac{x_{2}^{\prime *}}{x_{1}^{\prime *} x_{2}^{\prime}}\right)^{-n / 2}\left|\frac{x_{1}^{\prime}}{x_{2}^{\prime}}\right|^{2 i \nu}
\end{aligned}
$$

Integrating with respect to $x_{1}^{\prime}$ and $x_{2}^{\prime}$ leads to a lot of $\delta$ distributions since

$$
\frac{1}{2 \pi} \int \frac{d x_{1}^{\prime} d x_{1}^{\prime *}}{2 x_{1}^{\prime} x_{1}^{\prime *}} x_{1}^{\prime \frac{n_{1}-n}{2}-i\left(\nu_{1}-\nu\right)} x_{1}^{\prime *-\frac{n_{1}-n}{2}-i\left(\nu_{1}-\nu\right)}=\pi \delta_{n_{1}, n} \delta\left(\nu_{1}-\nu\right)
$$


and a similar integral over $x_{2}^{\prime}$. Defining $F_{\text {tot }\{n, \nu\}}^{(1)}\left(\underline{x}_{1}, \underline{x}_{2}\right)$ and $n_{\{n, \nu\}}\left(\underline{x}_{1}, \underline{x}_{2}\right)$ via

$$
F_{t o t\{n, \nu\}}^{(1)}\left(\underline{x}_{1}, \underline{x}_{2}\right)=\sum_{n=-\infty}^{+\infty} \int_{-\infty}^{+\infty} \frac{d \nu}{2 \pi} F_{t o t\{n, \nu\}}^{(1)}\left(\underline{x}_{1}, \underline{x}_{2}\right) \exp \left(\frac{2 \alpha_{s} N_{c}}{\pi} \chi(n, \nu) \tilde{Y}\right)
$$

and

$$
n_{\{n, \nu\}}\left(\underline{x}_{1}, \underline{x}_{2}\right)=\sum_{n=-\infty}^{+\infty} \int_{-\infty}^{+\infty} \frac{d \nu}{2 \pi} n_{\{n, \nu\}}\left(\underline{x}_{1}, \underline{x}_{2}\right) \exp \left(\frac{2 \alpha_{s} N_{c}}{\pi} \chi(n, \nu) \tilde{Y}\right)
$$

this finally yields

$$
\begin{aligned}
F_{t o t\{n, \nu\}}^{(1)}\left(\underline{x}_{1}, \underline{x}_{2}\right) & =-\frac{\pi \alpha_{s}^{2}}{4} \frac{1+(-1)^{n}}{\left(\nu^{2}+\left(\frac{n-1}{2}\right)^{2}\right)\left(\nu^{2}+\left(\frac{n+1}{2}\right)^{2}\right)}\left(\frac{x_{1}^{*} x_{2}}{x_{1} x_{2}{ }^{*}}\right)^{n / 2}\left|x_{1}\right|^{1+2 i \nu}\left|x_{2}\right|^{1-2 i \nu} \\
& =-\frac{1+(-1)^{n}}{8} \frac{1 \alpha_{s}^{2} x_{2}^{2}}{\left(\nu^{2}+\left(\frac{n-1}{2}\right)^{2}\right)\left(\nu^{2}+\left(\frac{n+1}{2}\right)^{2}\right)} n_{\{n, \nu\}}\left(\underline{x}_{1}, \underline{x}_{2}\right)
\end{aligned}
$$

Comparison with Eq. (2.58) provides a check of this calculation when integrating both sides with respect to $\underline{b}$. In the asymptotic regime, corresponding to $n=0$,

$$
F_{\{0, \nu\}}^{(1)}\left(\underline{x}_{1}, \underline{x}_{2}\right)=-\frac{\pi \alpha_{s}^{2} x_{2}^{2}}{4} \frac{1}{\left(\nu^{2}+\frac{1}{4}\right)^{2}} n_{\{0, \nu\}}\left(\underline{x}_{1}, \underline{x}_{2}\right),
$$

which could be obtained from Eq. (2.59). Using Eq. (2.65), the corresponding asymptotic integrated dipole distribution reads

$$
n\left(\underline{x}_{1}, \underline{x}_{2}, \tilde{Y}\right)=\int \frac{d \nu}{2 \pi} \frac{x_{1}}{x_{2}}\left(\frac{x_{1}}{x_{2}}\right)^{-2 i \nu} \exp \left(\frac{2 \alpha N_{c}}{\pi} \chi(n, \nu) \tilde{Y}\right),
$$

which gives, in the saddle point approximation at large $\tilde{Y}$,

$$
n\left(\underline{x}_{1}, \underline{x}_{2}, \tilde{Y}\right)=\frac{1}{2} \frac{x_{1}}{x_{2}} \frac{\exp \left\{\frac{4 \alpha_{s} N_{c}}{\pi} \ln 2 \tilde{Y}\right\}}{\sqrt{\frac{7}{2} \alpha_{s} N_{c} \zeta(3) \tilde{Y}}} \exp \left\{-\frac{\ln ^{2}\left(x_{1} / x_{2}\right)}{\frac{14 \alpha_{s} N_{c}}{\pi} \zeta(3) \tilde{Y}}\right\} .
$$

Thus, one gets for the total cross-section

$$
\begin{aligned}
F^{(1)}\left(\underline{x}_{1}, \underline{x}_{2}, \tilde{Y}\right) & \simeq-4 \pi \alpha_{s}^{2} x_{2}^{2} n\left(\underline{x}_{1}, \underline{x}_{2}, \tilde{Y}\right) \\
& \simeq-2 \pi \alpha_{s}^{2} x_{1} x_{2} \frac{\exp \left\{\frac{4 \alpha_{s} N_{c}}{\pi} \ln 2 \tilde{Y}\right\}}{\sqrt{\frac{7}{2} \alpha_{s} N_{c} \zeta(3) \tilde{Y}}} \exp \left\{-\frac{\ln ^{2}\left(x_{1} / x_{2}\right)}{\frac{14 \alpha_{s} N_{c}}{\pi} \zeta(3) \tilde{Y}}\right\},
\end{aligned}
$$

in agreement with formula (26) of Ref. 15.

Let us integrate the scattering amplitude $F^{(1)}(\underline{b})$ with respect to the impact parameter $\underline{b}$ in the domain (2.61) where formula (2.60) is valid (neglecting the fact that the upper bound is not infinite). The corresponding integration then gives

$$
F^{(1)}\left(\underline{x}_{1}, \underline{x}_{2}, \tilde{Y}\right) \simeq-2 \pi \alpha_{s}^{2} x_{1} x_{2} \frac{\exp \left\{\frac{4 \alpha_{s} N_{c}}{\pi} \ln 2 \tilde{Y}\right\}}{\sqrt{\frac{7}{2} \alpha_{s} N_{c} \zeta(3) \tilde{Y}}} \exp \left\{-\frac{\ln ^{2}\left(x_{1} / x_{2}\right)}{\frac{14 \alpha_{s} N_{c}}{\pi} \zeta(3) \tilde{Y}}\right\},
$$


which is identical to Eq. (2.74). From the gaussian distribution obtained in Eq. (2.75), it is clear, comparing with Eq. (2.60), that the total cross section at BFKL order is dominated by impact parameter configuration much larger than the transverse sizes of the two scattering onia, corresponding to

$$
\ln \left(\frac{16 b^{2}}{x_{1} x_{2}}\right) \sim \sqrt{\frac{14 \alpha_{s} N_{c}}{\pi} \zeta(3) \tilde{Y}} .
$$

Note that this dominant contribution is inside the domain (2.61). These dominant configurations are much more central than what was claimed in Ref. [16]. It confirms previous numerical simulations [27]. Thus, the calculation, based on perturbative QCD, is expected to remain valid for high values of $\tilde{Y}$.

\section{Electron-Onium Deep Inelastic Scattering}

In this section, we perform an analysis of $e^{ \pm}$-onium deep inelastic scattering at low $x_{b j}$, based on $k_{T}$-factorization and dipole color model, as illustrated in figure 2. Our aim is to compute

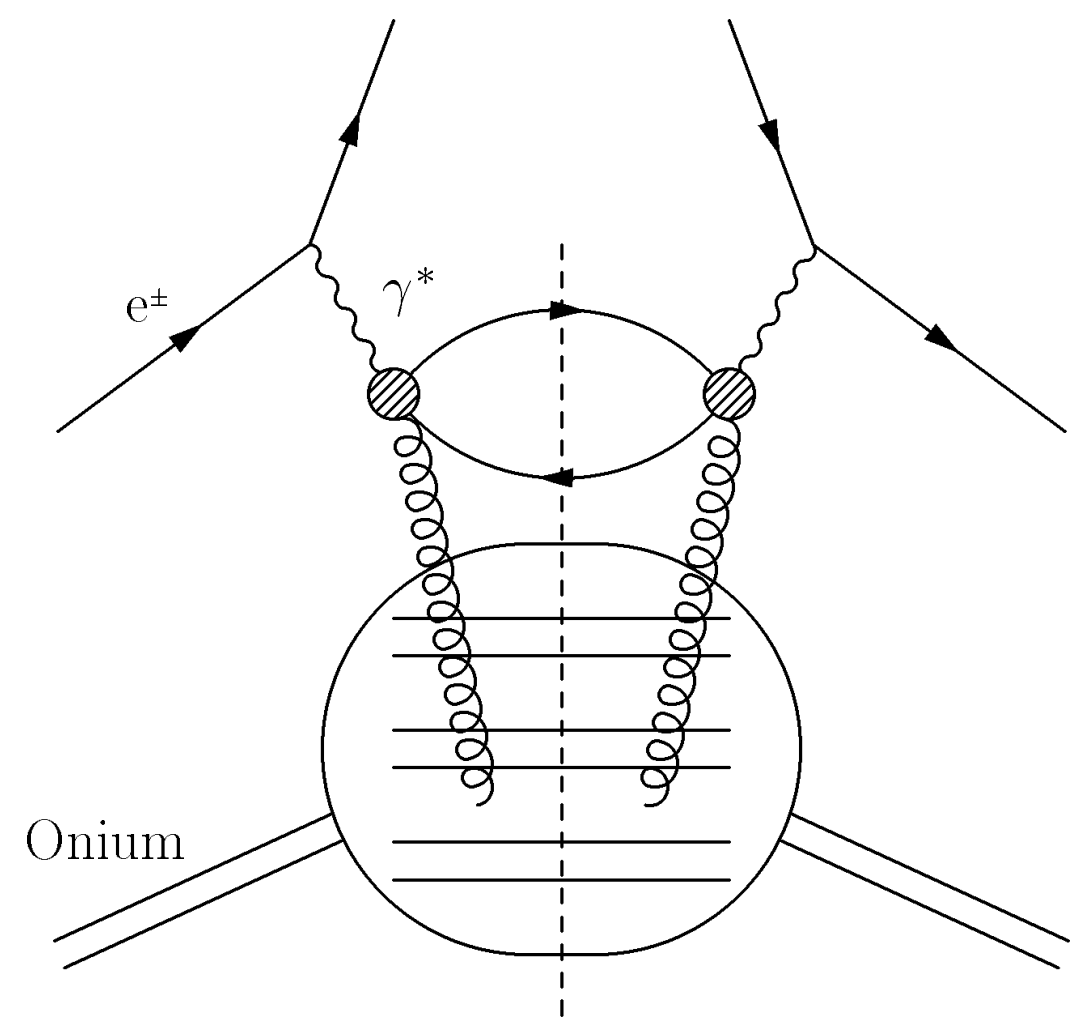

Figure 2: $k_{T}$-factorization and dipole model applied to $e^{ \pm}-$onium deep inelastic scattering.

various structure functions in the small $x_{b j}$ regime, where the onium wave function is dominated by a color dipole cascade.

In the Regge limit, one can apply the $k_{T}$-factorization tool [20, 21, 22] in order to extract a photon of virtuality $Q^{2}$ off an onium. It involves the elementary Born cross-section $\hat{\sigma}_{\gamma g} / Q^{2}$ of 
the process $\gamma g(k) \rightarrow q \bar{q}$. Here the gluon is off-shell, quasi transverse, with a virtuality $k^{2} \simeq \underline{k}^{2}$. One also has to introduce the unintegrated gluon distribution density at a factorization scale $Q_{0}^{2}$, which is related to the usual gluon distribution by

$$
G\left(x_{b j}, Q^{2}, Q_{0}^{2}\right)=\int_{0}^{Q^{2}} d^{2} \underline{k} \mathcal{F}\left(x_{b j}, \underline{k}, Q_{0}^{2}\right)
$$

We first deal with a dipole of transverse size $x_{01}$, which can be either part of a heavy onium (i.e. a heavy $q \bar{q}$ pair) or extracted from a proton as will be emphazied later. The $k_{T}$-factorization implies, for the total $\gamma^{*}-$ dipole cross- section $\sigma_{\gamma^{*}}^{d}$,

$$
Q^{2} \sigma_{\gamma^{*}}^{d}\left(x_{b j}, Q^{2} ; x_{01}^{2}\right)=\int d^{2} \underline{k} \int_{0}^{1} \frac{d z}{z} \hat{\sigma}_{\gamma g}\left(x_{b j} / z, \underline{k}^{2} / Q^{2}\right) \mathcal{F}\left(z, \underline{k} ; x_{01}^{2}\right)
$$

$\mathcal{F}\left(z, \underline{k} ; x_{01}^{2}\right)$ being the Fourier transform of $\mathcal{F}\left(z, \underline{k} ; Q_{0}^{2}\right)$ in transverse space. We next evaluate $\mathcal{F}$ by coupling the gluon to the softest dipole which arises in the cascade. This is achieved by using a second $k_{T}$-factorization. It involves the elementary Born cross-section $\hat{\sigma}_{g d} / k^{2}$ of the process $d(\underline{x}) g(k) \rightarrow d(\underline{x})$ for a dipole of transverse size $\underline{x}$ and a soft gluon of virtuality $\underline{k}^{2}$. This $k_{T}$-factorization can be expressed by

$$
\underline{k}^{2} \mathcal{F}\left(z, \underline{k} ; x_{01}^{2}\right)=\int \frac{d^{2} \underline{x}}{(2 \pi)^{2} \underline{x}^{2}} \int_{z}^{z_{1}} \frac{d z^{\prime}}{z^{\prime}} n\left(\underline{x}_{01}, \underline{x}, \ln \frac{z_{1}}{z^{\prime}}\right) \hat{\sigma}_{g d}\left(z / z^{\prime}, \underline{x}^{2} \underline{k}^{2}\right) \delta\left(z / z^{\prime}-1\right),
$$

where the distribution density $n\left(\underline{x}_{01}, \underline{x}, z\right)$ was defined in section 2 . As previously $z_{1} p_{+}$is the light-cone momentum of the quark part of the dipole $\left(\underline{x}_{01}\right) . \hat{\sigma}_{g d}$ is computed in appendix A.3 using eikonal techniques. Defining $\tilde{Y}=\ln z_{1} / z$, one gets for the dipole-photon cross-section

$$
\begin{aligned}
Q^{2} \sigma_{\gamma^{*}}^{d}\left(x_{b j}, Q^{2} ; x_{01}^{2}\right)=\int d^{2} \underline{k} \int_{0}^{1} & \frac{d z}{z} \hat{\sigma}_{\gamma g}\left(x_{b j} / z, \frac{\underline{k}^{2}}{Q^{2}}\right) \int \frac{d^{2} \underline{x}}{\underline{x}^{2}} n\left(\underline{x}_{01}, \underline{x}, \tilde{Y}\right) \\
& \times 4 \pi \alpha_{s} \frac{N_{c}}{(2 \pi)^{4}}\left(2-e^{i \underline{k} \cdot \underline{x}}-e^{-i \underline{k} \cdot \underline{x}}\right) \frac{1}{\underline{k}^{2}} .
\end{aligned}
$$

Let us now compute the convolutions in longitudinal and transverse spaces. As in section 2, we introduce a double Mellin-transform in these both variables, namely

$$
n\left(\underline{x}_{01}, \underline{x}, \tilde{Y}\right)=\int \frac{d \omega}{2 i \pi} e^{\omega \tilde{Y}} n_{\omega}\left(\underline{x}_{01}, \underline{x}\right)
$$

and

$$
n_{\omega}\left(\underline{x}_{01}, \underline{x}\right)=\int \frac{d \gamma}{2 i \pi}\left(\frac{x_{01}}{x}\right)^{2 \gamma} n_{\omega}(\gamma) .
$$

Here the Mellin variable in transverse space is $\gamma=\frac{1}{2}+i \nu$, which is introduced here rather than $\nu$ since it plays the role of an anomalous dimension. We consider only the dominant Regge trajectory, that is $n=0$. Formula (2.17) then reads

$$
n_{\omega}(\gamma)=\frac{2}{\omega-\frac{2 \alpha_{s} N_{c}}{\pi} \chi(\gamma)}
$$

where

$$
\chi(\gamma)=\chi\left(0, \frac{1}{2}+i \nu\right)=\Psi(1)-\frac{1}{2} \Psi(\gamma)-\frac{1}{2} \Psi(1-\gamma)
$$


The quantity $\hat{\sigma}_{\gamma g}$ has been calculated for different polarizations of the incoming photon in [20]. We introduce the corresponding double Mellin transform of this cross-section, namely

$$
4 \pi^{2} \alpha_{e . m} h_{\omega}(\gamma)=\gamma \int_{0}^{\infty} \frac{d \underline{k}^{2}}{\underline{k}^{2}}\left(\frac{\underline{k}^{2}}{Q^{2}}\right)^{\gamma} \hat{\sigma}_{\omega}\left(\frac{\underline{k}^{2}}{Q^{2}}\right)
$$

or equivalently

$$
\hat{\sigma}_{\omega}\left(\frac{l^{2}}{Q^{2}}\right)=4 \pi^{2} \alpha_{e . m} \int \frac{d \gamma}{2 i \pi}\left(\frac{l^{2}}{Q^{2}}\right)^{-\gamma} \frac{1}{\gamma} h_{\omega}(\gamma)
$$

with

$$
\hat{\sigma}_{\omega}\left(\frac{\underline{k}^{2}}{Q^{2}}\right)=\int_{0}^{1} d z z^{\omega-1} \hat{\sigma}\left(z, \frac{\underline{k}^{2}}{Q^{2}}\right) .
$$

The expression for $\sigma_{\gamma^{*}}^{d}$ now reads, after performing the convolution in longitudinal space,

$$
\begin{gathered}
Q^{2} \sigma_{\gamma^{*}}^{d}\left(x_{b j}, Q^{2} ; x_{01}^{2}\right)=4 \pi^{2} \alpha_{e . m} \frac{\alpha_{s} N_{c}}{4 \pi^{3}} \int d^{2} \underline{k} \int \frac{d \gamma^{\prime}}{2 i \pi} \int \frac{d \gamma}{2 i \pi} \int \frac{d \omega}{2 i \pi} \exp \left(\omega \ln \frac{z_{1}}{x_{b j}}\right)\left(\frac{x_{01}}{x}\right)^{2 \gamma} \\
\times \frac{2}{\omega-\frac{2 \alpha_{s} N_{c}}{\pi} \chi(\gamma)} \frac{h_{\omega}\left(\gamma^{\prime}\right)}{\gamma^{\prime}} \int \frac{d^{2} \underline{x}}{\underline{x}^{2}}\left(\frac{\underline{k}^{2}}{Q^{2}}\right)^{-\gamma^{\prime}}\left(2-e^{i \underline{k} \cdot \underline{x}}-e^{-i \underline{k} \cdot \underline{x}}\right) \frac{1}{\underline{k}^{2}} .
\end{gathered}
$$

The integration with respect to the polar angle of $\underline{x}$ leads to a Bessel function. One has then to integrate over $x$, namely

$$
\int \frac{d x}{x} 4 \pi\left(1-J_{0}(k x)\right)\left(\frac{x}{x_{01}}\right)^{-2 \gamma}=4 \pi\left(k x_{01}\right)^{2 \gamma} \frac{2^{-1-2 \gamma}}{\gamma} \frac{\Gamma(1-\gamma)}{\Gamma(1+\gamma)} \equiv 4 \pi\left(k x_{01}\right)^{2 \gamma} v(\gamma) .
$$

The integration over $k$ gives $\gamma=\gamma^{\prime}$. Since $n_{\omega}(\gamma)$ (formula (3.83)) exhibits a pole at $\omega_{p}=$ $\frac{\alpha_{s} N_{c}}{\pi} \chi(\gamma)$, the $\omega$ integral finally yields

$$
\frac{Q^{2}}{4 \pi^{2} \alpha_{e . m}} \sigma_{\gamma^{*}}^{d}\left(x_{b j}, Q^{2} ; x_{01}^{2}\right)=\frac{2 \alpha_{s} N_{c}}{\pi} \int \frac{d \gamma}{2 i \pi} h_{\omega_{p}}(\gamma) \frac{v(\gamma)}{\gamma}\left(\underline{x}_{01}^{2} Q^{2}\right)^{\gamma} \exp \left(\frac{2 \alpha_{s} N_{c}}{\pi} \chi(\gamma) \ln \frac{z_{1}}{x_{b j}}\right)
$$

In the regime we are interested in, $\omega_{p} \ll \gamma$ and the dependence of $h_{\omega_{p}}(\gamma)$ on $\omega_{p}$ can be neglected, replacing $\omega_{p}$ by 0 [20]. We then get for the total cross-section $\gamma^{*}-\operatorname{dipole}\left(\underline{x}_{01}\right)$ and for the related structure function (we assume $R=F_{L} / F_{T}$ to be small in the relation between the total crosssection and the structure function)

$$
\frac{Q^{2}}{4 \pi^{2} \alpha_{e . m}} \sigma_{\gamma^{*}}^{d}\left(x_{b j}, Q^{2} ; x_{01}^{2}\right)=F_{\gamma}^{d}\left(x_{b j}, Q^{2} ; x_{01}^{2}\right)=\frac{2 \alpha_{s} N_{c}}{\pi} \int \frac{d \gamma}{2 i \pi}\left(Q^{2} x_{01}^{2}\right)^{\gamma} h(\gamma) \frac{v(\gamma)}{\gamma} e^{\frac{\alpha_{s} N_{c}}{\pi} \chi(\gamma) \ln \frac{1}{x_{b j}}}
$$

The dipole $\left(\underline{x}_{01}\right)$ being part of a bound state, for example extracted from an onium, one has now to average with respect to the coresponding wave function, $\Phi^{(0)}\left(\underline{x}_{01}, z_{1}\right)$. The initial dipole state is supposed to be well localized in transverse space. Namelly, its transverse size, given by the scale $M^{2}$ which is defined via

$$
\left(M^{2}\right)^{-\gamma}=\int d^{2} \underline{x}_{01}\left(x_{01}^{2}\right)^{\gamma} d z_{1} \Phi^{(0)}\left(\underline{x}_{01}, z_{1}\right),
$$

is assumed to be perturbative. One then obtains for the onium structure function

$$
F^{\mathcal{O} \text { nium }}\left(x_{b j}, Q^{2} ; M^{2}\right)=2 \frac{\alpha_{s} N_{c}}{\pi} \int \frac{d \gamma}{2 i \pi} h(\gamma) \frac{v(\gamma)}{\gamma}\left(\frac{Q^{2}}{M^{2}}\right)^{\gamma} \exp \left(\frac{2 \alpha_{s} N_{c}}{\pi} \chi(\gamma) \ln \frac{1}{x_{b j}}\right)
$$


It is then possible to apply formula (3.93) to peculiar structure functions, namely

$$
\left(\begin{array}{c}
F_{T} \\
F_{L} \\
F_{G}
\end{array}\right)=\frac{2 \alpha_{s} N_{c}}{\pi} \int \frac{d \gamma}{2 i \pi}\left(\frac{Q^{2}}{M^{2}}\right)^{\gamma} \exp \left(\frac{2 \alpha_{s} N_{c}}{\pi} \chi(\gamma) \ln \frac{1}{x_{b j}}\right)\left(\begin{array}{c}
h_{T} \\
h_{L} \\
1
\end{array}\right) \frac{v(\gamma)}{\gamma}
$$

where $F_{T(L)}$ is the structure function corresponding to transverse (longitudinal) photons and $F_{G}$ the gluon structure function. The coefficient functions

$$
\left(\begin{array}{c}
h_{T} \\
h_{L}
\end{array}\right)=\frac{\alpha_{s}}{3 \pi \gamma} \frac{(\Gamma(1-\gamma) \Gamma(1+\gamma))^{3}}{\Gamma(2-2 \gamma) \Gamma(2+2 \gamma)} \frac{1}{1-\frac{2}{3} \gamma}\left(\begin{array}{c}
(1+\gamma)\left(1-\frac{\gamma}{2}\right) \\
\gamma(1-\gamma)
\end{array}\right)
$$

were computed in ref [20]. When $x_{b j}$ is small, the $\gamma$-integration can be performed by the steepest-descent method. The corresponding asymptotic saddle point is located at $\gamma=\frac{1}{2}$, which defines the BFKL anomalous dimension. Expanding the $\chi$ function around $\frac{1}{2}$, we obtain a saddle point at

$$
\gamma_{s}=\frac{1}{2}\left(1-a \ln \frac{Q}{Q_{0}}\right), \quad \text { where } \quad a=\left(\frac{\bar{\alpha} N_{c}}{\pi} 7 \zeta(3) \ln \frac{1}{x_{b j}}\right)^{-1} .
$$

The approximation of expanding $\chi(\gamma)$ around $\frac{1}{2}$ is valid when

$$
a \ln \left(\frac{Q}{M}\right) \simeq \ln \frac{Q}{M} / \ln \frac{1}{x_{b j}} \ll 1
$$

that is the small $x_{b j}$, moderate $Q / M$ kinematical domain. This yields

$$
F_{2} \equiv F_{T}+F_{L}=C a^{1 / 2} \frac{Q}{M} \exp \left(\left(\alpha_{P}-1\right) \ln \frac{1}{x_{b j}}-\frac{a}{2} \ln ^{2} \frac{Q}{Q_{0}}\right)
$$

where $\alpha_{P}$ is defined by Eq. (1.2). Thus, $F_{2}$ depends only on 3 parameters, $C, M$ and $\alpha_{P}$. Suppose we can fit $F_{2}$ with this form. Then, get a prediction for $F_{G}$ and $R=F_{L} / F_{T}$ without any free parameter. Namely,

$$
\frac{F_{G}}{F_{2}}=\left.\frac{1}{h_{T}+h_{L}}\right|_{\gamma=\gamma_{s}} \equiv \frac{3 \pi \gamma_{s}}{\alpha_{s}} \frac{1-\frac{2}{3} \gamma_{s}}{1+\frac{3}{2} \gamma_{s}-\frac{3}{2} \gamma_{s}^{2}} \frac{\Gamma\left(2-2 \gamma_{s}\right) \Gamma\left(2+2 \gamma_{s}\right)}{\left(\Gamma\left(1-\gamma_{s}\right) \Gamma\left(1+\gamma_{s}\right)\right)^{3}}
$$

and

$$
R=\frac{h_{L}}{h_{T}}\left(\gamma_{s}\right)=\frac{\gamma_{s}\left(1-\gamma_{s}\right)}{\left(1+\gamma_{s}\right)\left(1-\frac{\gamma_{s}}{2}\right)}
$$

where $\gamma_{s}$ is given by the expression (3.96). Note that the overall non-perturbative normalization $C$ does not enter $R$ and $F_{G} / F_{2}$. It is possible to apply this analysis to the proton [24]. This requires some additional asumptions when considering the coupling of the dipole cascade to the proton. It leads to a successful description of the HERA data [1]. It also provides a prediction for the gluon density based on the BFKL dynamics, and a prediction for the ratio $R$, using formulas (3.99) and (3.100). All the previous annalysis was done by performing an expansion around the small $x_{b j}$ behaviour of the BFKL Pomeron, that is by considering $\ln 1 / x_{b j}$ as a big parameter, which leads to a saddle point close to $\gamma=\frac{1}{2}$ (see Eq. (3.96)). A different analysis of the modification, due to the BFKL dynamics, of the anomalous dimension of the double 
logarithmic approximation common to DGLAP and BFKL can be done by considering now $\ln Q^{2} / M^{2}$ as a big parameter. The corresponding saddle point of Eq. (3.94) is now around $\gamma=0$. In Mellin space, the double logarithmic expression of the anomalous dimension is given by $\gamma_{\omega}\left(\alpha_{s}\right)=3 \alpha_{s} /(\pi \omega)$, and expansion of the $\chi$ function (3.84) around $\gamma=0$ leads to corrections given by powers of $3 \alpha_{s} /(\pi \omega)$. This method provides an extension of the domain of applicability (3.97) [36.

As it has been seen in this section, the dipole model can be safely applied when the two scales of the process are both perturbative, as it is the case for $e^{ \pm}$- onium scattering. The application to $e^{ \pm}-p$ scattering requires some assumptions for the coupling to the proton. Because of the well-known diffusion in transverse momentum space, such an application of the dipole model, although successful [23, 24, 25], cannot be considered as a clean test of highenergy perturbative regge dynamics. A possible test of such dynamics could be based on single jets events in DIS [37] or double jets events in hadron-hadron collision [38]. Another interesting test of BFKL dynamics would be the $\gamma^{*}-\gamma^{*}$ events in $e^{+}-e^{-}$colliders at high energy in the center of mass of the virtual photon pair and with high (perturbative) photons virtualities. This has been already proposed in the framework of the original BFKL equation [39]. Such a process can equivalently be described in the dipole picture of BFKL dynamics. This will be developped elsewhere 40].

\section{Conclusion}

In this article we have shown the exact equivalence between BFKL and dipole approaches for the onium-onium cross-section at fixed impact parameter. This proof relies on conformal properties of the dipole cascade and of the elementary dipole-dipole cross section. We have also obtained asymptotic expressions for the dipole distribution inside an onium and for the oniumonium cross-section at fixed impact parameter. These results agrees with previous numerical simulations. We also apply the dipole model to onium- $e^{ \pm}$deep inelastic scattering, using the $k_{T^{-}}$ factorization, and obtain predictions for various structure functions in the BFKL dynamics. The different elementary cross-sections used in this paper are computed using eikonal techniques.

Relying on the same conformal properties, it should be possible to get analytical expressions for the multipomeronic contributions to the onium-onium cross-section, which are expected to be important for large rapidities.

From a phenomenological point of vue, the dipole framework could be applied to other inclusive processes. The application of this technique for exclusive quantities remains however an open question, due to the use of light-cone quantization, in which the intermediate states are unphysical.

\section{Acknowledgements:}

We thank Robi Peschanski for discussions, and André Morel for a careful reading of the manuscript and many fruitful comments. S.W wishes to thank Hans Lotter, Jochen Bartels and Gregory Korchemsky for comments. He warmly thanks Al Mueller for many illuminating discussions in Columbia and in Orsay. He thanks the Alexander von Humboldt Foundation and the II. Institüt für Theoretische Physik at DESY for support. 


\section{A Appendices}

\section{A.1 Calculation of $\sigma_{D D}$ using eikonal methods}

In this appendix we compute the dipole-dipole cross-section using eikonal techniques. In QCD, the eikonal current due to a fast quark of momentum $p$, responsible for the emission of a soft gluon of momentum $k(k \ll p)$ and color $a$ reads

$$
j^{\nu}(k)=-i g T^{a} \frac{p^{\nu}}{p \cdot k+i \epsilon} .
$$

Let us consider the scattering of two dipoles. Let $\underline{x}_{0}\left(\underline{x}_{1}\right)$ be the transverse coordinate of the free quark (antiquark) making the right moving dipole and $\underline{x}_{0}^{\prime}\left(\underline{x}_{1}^{\prime}\right)$ the coordinates of the corresponding quark (antiquark) making the left moving dipole. In this mixed representation where the fast radiating particule is represented in impact parameter space, and the radiated gluon is represented in momentum space, the eikonal current corresponding to a quark of transverse coordinate $\underline{x}$ then reads

$$
j^{a \nu}(k)=-i g T^{a} \frac{\bar{\eta}^{\nu}}{\bar{\eta} \cdot k+i \epsilon} e^{-i \underline{x} \cdot \underline{k}},
$$

where $\bar{\eta}=(\stackrel{+}{1}, \overline{0}, \stackrel{\perp}{0})$. This current is responsible for a term $-i j^{a \nu}(k) \epsilon_{\nu}$ when computing the amplitude of emission of a gluon by the quark. This terms arises when evaluating the evolution operator $T \exp -i \int d^{4} x j^{a \nu}(x) A_{a \nu}(x)$ due to the usual hamiltonian $\int d^{3} x j^{a \nu}(x) A_{a \nu}(x)$. Note that this current is completely described by the transverse size of the dipole and does not require any additional information about its internal structure.

The eikonal current corresponding to the right moving dipole then reads

$$
j^{a \nu}(k)=-i g T^{a} \frac{\bar{\eta}^{\nu}}{\bar{\eta} \cdot k+i \epsilon}\left(e^{-i \underline{x}_{0} \cdot \underline{k}}-e^{-i \underline{x}_{1} \cdot \underline{k}}\right),
$$

and a corresponding formula for the left moving dipole, replacing $\bar{\eta}$ by $\eta=(\stackrel{+}{0}, \overline{1}, \stackrel{\perp}{0})$.

Let us now compute the graph $A_{1}$ represented in figure 3. It reads, in Feynman covariant gauge,

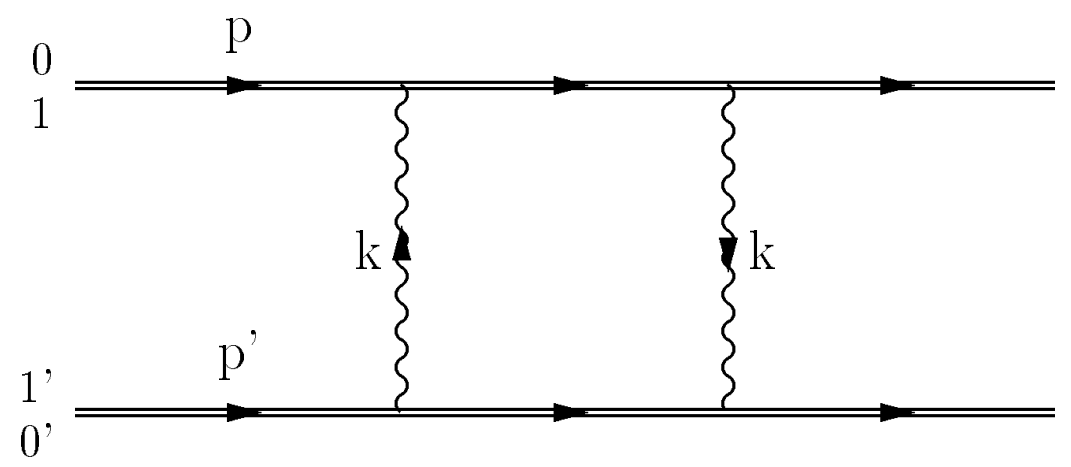

Figure 3: Contribution to the dipole-dipole scattering.

$$
\begin{aligned}
& A_{1}=\frac{1}{N_{c}^{2}} \sum_{a b} \frac{1}{2 p^{+}} \frac{1}{2 p^{\prime}-} \int \frac{d^{4} k}{(2 \pi)^{4}}\left\{\frac{2(-p \cdot k)}{i}\left(-i j^{b+}(-k)\right)\right\}\left\{\frac{2\left(p^{\prime} \cdot k\right)}{i}\left(-i j^{b-}(k)\right)\right\} \\
& \times\left(-i j^{b+}(k)\right)\left(-i j^{b-}(-k)\right) \frac{(-i)^{2}}{\left(k^{2}\right)^{2}} .
\end{aligned}
$$


The terms $\frac{1}{2 p^{+}} \frac{1}{2 p^{-}}$are related to the normalisation of the initial dipole states and $\frac{2(-p \cdot k)}{i}$ and $\frac{2\left(p^{\prime} \cdot k\right)}{i}$ are due to the fact that the considered amplitude is computed for amputed propagators. The color factor reads

$$
\frac{1}{N_{c}^{2}} \sum_{a b} \operatorname{Tr} T^{a} T^{b} \operatorname{Tr} T^{a} T^{b}=\frac{N_{c}^{2}-1}{4 N_{c}^{2}},
$$

which equals $\frac{1}{4}$ in the large $N_{c}$ limit.

Using the expression (A.3), this yields

$$
\begin{aligned}
& A_{1}=\frac{g^{4}}{4} \int \frac{d^{4} l}{(2 \pi)^{4}} \frac{1}{\left(k^{2}\right)^{2}} \frac{1}{k^{-}+i \epsilon} \frac{1}{k^{+}-i \epsilon} \\
& \times\left(e^{i \underline{x}_{0} \cdot \underline{k}}-e^{i \underline{x}_{1} \cdot \underline{k}}\right)\left(e^{-i \underline{x_{0}} \cdot \underline{k}}-e^{-i \underline{x}_{1} \cdot \underline{k}}\right)\left(e^{-i \underline{x}_{0}^{\prime} \cdot \underline{k}}-e^{-i \underline{x}_{1}^{\prime} \cdot \underline{k}}\right)\left(e^{i \underline{x}_{0}^{\prime} \cdot \underline{k}}-e^{i \underline{x}_{1}^{\prime} \cdot \underline{k}}\right) .
\end{aligned}
$$

One has also to consider the crossed diagram $A_{2}$. In order to integrate with respect to $k^{-}$and $k^{+}$we consider two equivalent representations of $A_{1}$ and $A_{2}$, obtained by changing the sign of $k$ (see figure 4 . Forgetting the phase factor for a moment, the forward amplitude $A$ then reads

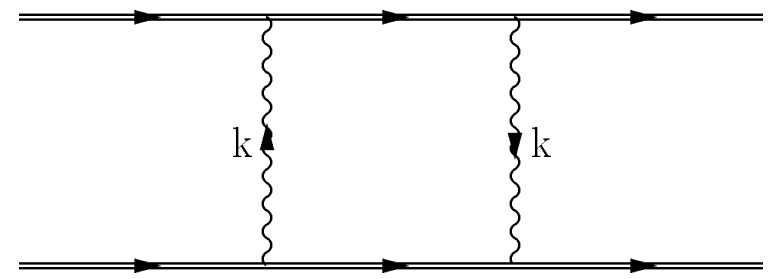

$A_{1}$

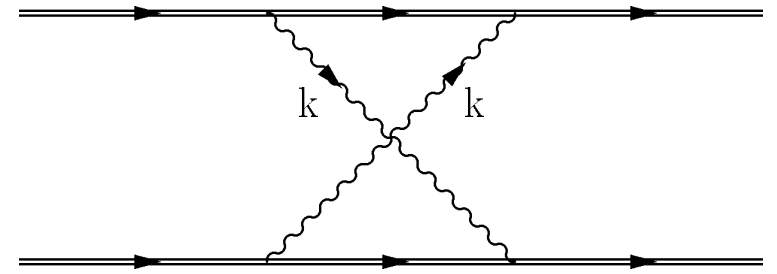

$A_{2}$

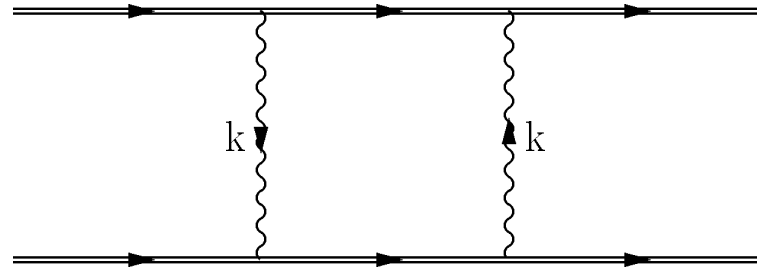

$A_{1}$

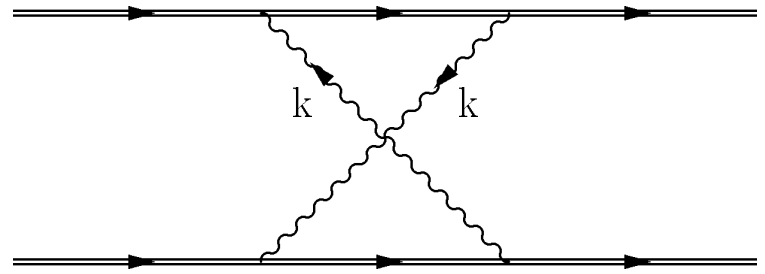

$A_{2}$

Figure 4: Contributions to the elementary dipole-dipole scattering.

$$
A=A_{1}+A_{2}=\frac{g^{4}}{4} \int \frac{d^{2} \underline{k}}{(2 \pi)^{2}} \frac{d k^{-}}{2 \pi} \frac{d k^{+}}{2 \pi} \frac{1}{\left(k^{2}\right)^{2}} \frac{1}{2}\left(\frac{1}{k^{-}+i \epsilon}+\frac{1}{-k^{-}+i \epsilon}\right)\left(\frac{1}{k^{+}+i \epsilon}+\frac{1}{-k^{+}+i \epsilon}\right) .
$$

The two last terms reads $\left(-2 \pi i \delta\left(k^{-}\right)\right)\left(-2 \pi i \delta\left(k^{+}\right)\right)$, which finally yield, performing the integration with respect to $k^{-}$and $k^{+}$

$$
A=-\frac{\alpha_{s}^{2}}{2} \int \frac{d^{2} \underline{k}}{\left(\underline{k}^{2}\right)^{2}}\left(2-e^{i \underline{k}} \cdot \underline{x}_{01}-e^{-i \underline{k}} \cdot \underline{x}_{01}\right)\left(2-e^{i \underline{k}} \cdot \underline{x}_{01}^{\prime}-e^{-i \underline{k} \cdot \underline{x}_{01}^{\prime}}\right),
$$

which was obtained in Ref. [15 by computing elementary Feynman diagrams. If we neglect the dependence of $\Phi\left(x_{01}, z\right)$ and $n\left(x_{01}, x, Y\right)$ with respect to the dipoles orientation (see appendix A.5), it is possible to average with respect to the angle of these dipoles. $A$ then reads

$$
A=-4 \pi \alpha_{s}^{2} \int_{0}^{\infty} \frac{d k}{k^{3}}\left[1-J_{0}\left(k x_{01}\right)\right]\left[1-J_{0}\left(k x_{01}^{\prime}\right)\right]
$$


Using the identity

$$
\int_{0}^{\infty} \frac{d k}{k^{3}}\left[1-J_{0}\left(k x_{01}\right)\right]\left[1-J_{0}\left(k x_{01}^{\prime}\right)\right]=\frac{1}{4} x_{<}^{2}\left[1+\ln \frac{x_{>}}{x_{<}}\right],
$$

where $x_{<}=\operatorname{Min}\left(x_{01}, x_{01}^{\prime}\right)$ and $x_{>}=\operatorname{Max}\left(x_{01}, x_{01}^{\prime}\right)$, this finally yields for the corresponding cross-section

$$
\sigma_{D D}\left(x_{01}, x_{01}^{\prime}\right)=-2 A=2 \pi \alpha_{s}^{2} x_{<}^{2}\left[1+\ln \frac{x_{>}}{x_{<}}\right] .
$$

This average result is however sufficient when computing the total cross-section (see appendix A.5).

Another representation of this elementary cross-section is very useful. Consider

$$
I=\frac{\alpha_{s}^{2}}{2} \int_{-\infty}^{+\infty} \frac{d \nu}{\left(\nu^{2}+\frac{1}{4}\right)^{2}} x_{1}^{1+2 i \nu} x_{2}^{1-2 i \nu}
$$

When $x_{1}>x_{2}\left(x_{1}<x_{2}\right)$ one can close the integration contour around $+i \infty(-i \infty)$, so that one pick up the (simple) pole at $\nu=i / 2(\nu=-i / 2)$. Thus,

$$
I=\left.\frac{\alpha_{s}^{2}}{2} 2 i \pi \frac{d}{d \nu} \frac{e^{2 i \nu \ln \frac{x}{x_{<}}}}{\left(\nu \pm \frac{i}{2}\right)^{2}}\right|_{\nu= \pm \frac{i}{2}} x_{>} x_{<}
$$

where $x_{<}=\operatorname{Min}\left(x_{1}, x_{2}\right)$ et $x_{>}=\operatorname{Max}\left(x_{1}, x_{2}\right)$. This finally yields the expected result:

$$
\sigma_{D D}\left(x_{1}^{\prime}, x_{2}^{\prime}\right)=\frac{\alpha_{s}^{2}}{2} \int_{-\infty}^{+\infty} \frac{d \nu}{\left(\nu^{2}+\frac{1}{4}\right)^{2}}\left(x_{1}^{\prime}\right)^{1+2 i \nu}\left(x_{2}^{\prime}\right)^{1-2 i \nu} .
$$

Note that this can be written equivalently as (see the following section)

$$
\sigma_{D D}\left(x_{1}^{\prime}, x_{2}^{\prime}\right)=\frac{\alpha_{s}^{2} x_{1}^{\prime} x_{2}^{\prime}}{4} \int_{-\infty}^{+\infty} \frac{d \nu}{\left(\nu^{2}+\frac{1}{4}\right)^{2}} \lim _{q \rightarrow 0}\left[E_{q}^{0 \nu *}\left(x_{1}^{\prime}\right) E_{q}^{0 \nu}\left(x_{2}^{\prime}\right)\right] .
$$

\section{A.2 Calculation of $\sigma_{D D}\left(\underline{x}, \underline{x}^{\prime}, \underline{b}-\underline{b}^{\prime}\right)$}

In this appendix we compute the elementary dipole-dipole cross-section at fixed impact parameter. As in the appendix A.1, we consider two dipoles of transverse sizes $\underline{x}=\underline{x}_{0}-\underline{x}_{1}$ and $\underline{x}^{\prime}=\underline{x}_{1}^{\prime}-\underline{x}_{0}^{\prime}$, whose centers are situated at $\underline{b}=\frac{\underline{x}_{0}+\underline{x}_{1}}{2}$ and $\underline{b}^{\prime}=\frac{\underline{x}_{0}^{\prime}+\underline{x}_{1}^{\prime}}{2}$.

Let us compute the non-forward scattering amplitude of these two dipoles. In the highenergy limit $-t<<s=2 p \cdot p^{\prime}$, the exchanged momentum is quasi-transverse

$$
q \simeq(\stackrel{+}{0}, \overline{0}, \underline{\perp})
$$

The graphs to be computed are displayed in figure 5. These graphs are computed using the rules of appendix A.1. Since $q^{-}=q^{+}=0$, the expression for the corresponding amplitude is similar to the one obtained in equation (A.8), except for phase factors. It reads

$$
\begin{aligned}
A=-\frac{\alpha_{s}^{2}}{2} \int & \frac{d^{2} \underline{q}}{(2 \pi)^{2}} \int \frac{d^{2} \underline{k}}{\underline{k}^{2}(\underline{k}+\underline{q})^{2}}\left(e^{i \underline{x}_{0} \cdot \underline{k}}-e^{i \underline{x}_{1} \cdot \underline{k}}\right)\left(e^{-i \underline{x}_{0} \cdot(\underline{k}+\underline{q})}-e^{-i \underline{x}_{1} \cdot(\underline{k}+\underline{q})}\right) \\
& \times\left(e^{-i \underline{x}_{0}^{\prime} \cdot \underline{k}}-e^{-i \underline{x}_{1}^{\prime} \cdot \underline{k}}\right)\left(e^{i \underline{x}_{0}^{\prime} \cdot(\underline{k}+\underline{q})}-e^{i \underline{x}_{1}^{\prime} \cdot(\underline{k}+\underline{q})}\right)
\end{aligned}
$$




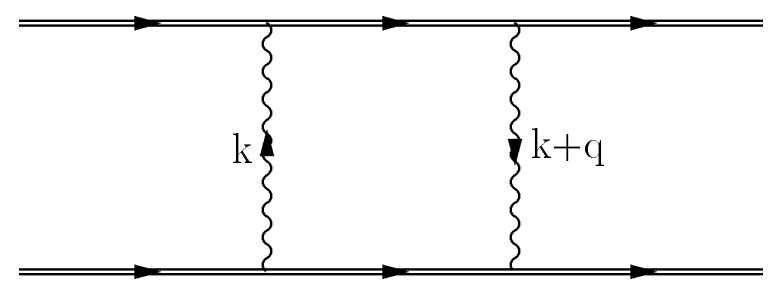

$A_{1}$

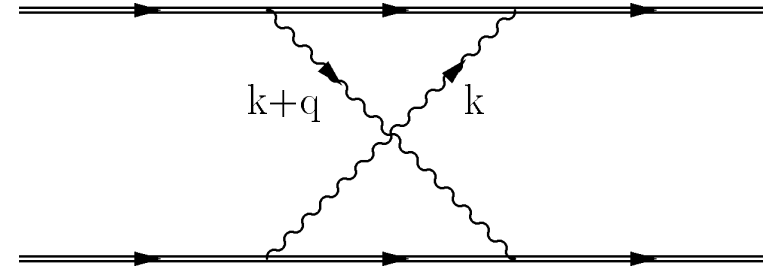

$A_{2}$

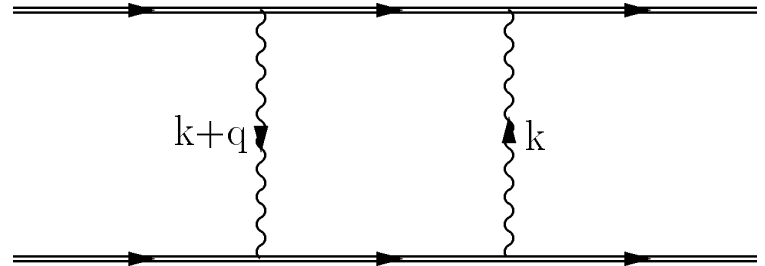

$A_{1}$

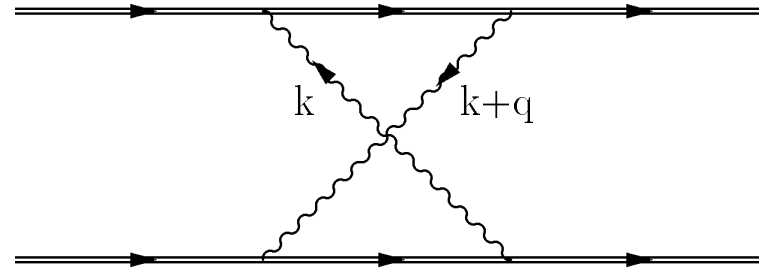

$A_{2}$

Figure 5: Contributions to the elementary dipole-dipole scattering with non zero exchanged momentum.

Note that this expression is overall translationally invariant. However, it will depend on the relative distance between the two dipoles and on their angles. Expanding the previous expression and integrating with respect to the angles of $\underline{k}$ and $\underline{k}+\underline{q}$, one obtains several Bessel functions

$$
A=-\frac{\alpha_{s}^{2}}{2}\left\{I\left(\left|\underline{x}_{0}-\underline{x}_{0^{\prime}}\right|\right)-I\left(\left|\underline{x}_{0}-\underline{x}_{1^{\prime}}\right|\right)-I\left(\left|\underline{x}_{1}-\underline{x}_{0^{\prime}}\right|\right)+I\left(\left|\underline{x}_{1}-\underline{x}_{1^{\prime}}\right|\right\}^{2},\right.
$$

where

$$
I(\underline{x})=\int_{\rho}^{+\infty} \frac{d k}{k} J_{0}(k x) .
$$

$\rho$ is an infrared cut-off which regularize the divergency at $k=0$. In the limit $\rho \rightarrow 0, I$ can be computed (see Ref. [14)

$$
I=\lim _{\lambda \rightarrow 0}\left[\int_{0}^{+\infty} \frac{d k}{k^{1-\lambda}} J_{0}(k x)-\int_{0}^{\rho} \frac{d k}{k^{1-\lambda}} J_{0}(k x)\right]=\psi(1)+\ln 2-\ln x-\ln \rho .
$$

When evaluating $A$, the constant $\psi(1)+\ln 2$ and the infrared divergent term $\ln \rho$ cancels. $A$ then reads

$$
A=-\frac{\alpha_{s}^{2}}{2}\left\{\ln \frac{\left|\underline{x}_{0}-\underline{x}_{0^{\prime}}\right|\left|\underline{x}_{1}-\underline{x}_{1^{\prime}}\right|}{\left|\underline{x}_{0}-\underline{x}_{1^{\prime}}\right| \underline{x}_{1}-\underline{x}_{0^{\prime}} \mid}\right\}^{2}
$$

Defining

$$
\underline{b}=\frac{\underline{x}_{0}+\underline{x}_{1}}{2} \quad \text { and } \quad \underline{b}^{\prime}=\frac{\underline{x}_{0^{\prime}}+\underline{x}_{1^{\prime}}}{2}
$$

the non-forward cross-section finally reads

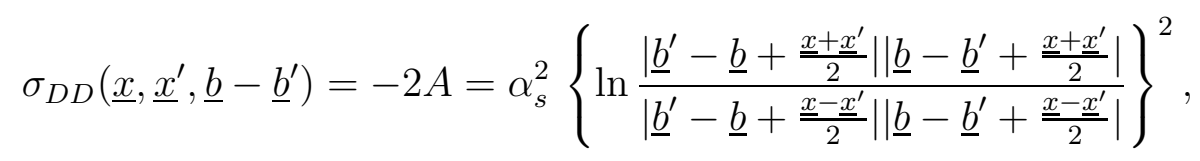

as quoted in Ref. [27]. Note that it only depends on the relative distance $\underline{b}-\underline{b}^{\prime}$ because of the overall translational invariance. This expression can also be obtained by evaluating 
this scattering amplitude in the laboratory frame of one of the two onia. In this frame, this amplitude is expressed as an eikonal phase (computed in terms of a Wilson-loop), due to the change of the wave function of the slow moving onium in the color field of the fast moving onium [26]. Let us now show that this cross-section can be expanded on the basis of the functions $E^{n, \nu}$. In the standard Regge calculation, instead of considering the scattering of two dipoles, one considers the scattering of two gluons of momenta $-\underline{k}$ and $\underline{k}+\underline{q}$ (see figure A.24). In the

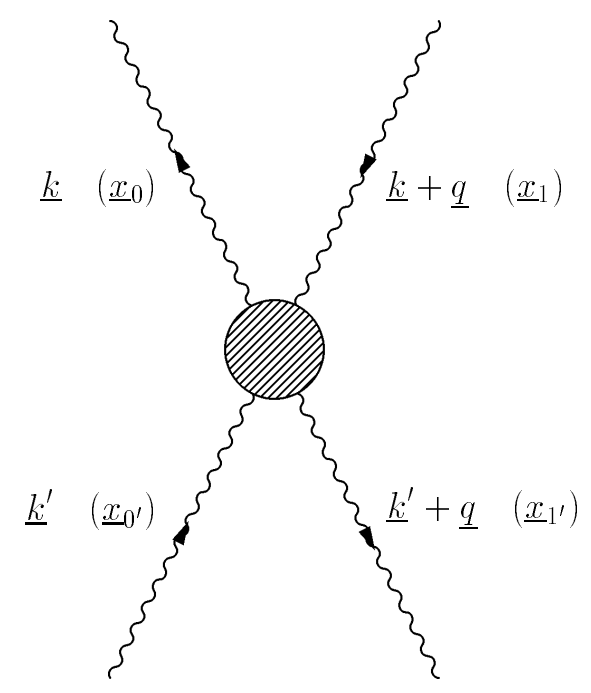

Figure 6: Gluon-Gluon diffusion in the Regge framework.

lowest order approximation, the contribution of this graph is

$$
A_{g g}=\frac{\delta^{2}\left(\underline{k}-\underline{k}^{\prime}\right)}{\underline{k}^{2}(\underline{k}+\underline{q})^{2}},
$$

which gives, after Fourier transform in impact parameter space 41]

$$
a\left(\underline{x}_{0}, \underline{x}_{1}, \underline{x}_{0^{\prime}}, \underline{x}_{1^{\prime}}\right)=(2 \pi)^{2} \ln \left(\left|\underline{x}_{00^{\prime}}\right| \lambda\right) \ln \left(\left|\underline{x}_{11^{\prime}}\right| \lambda\right)
$$

where $\lambda$ is the mass of the gluon introduced in order to remove the infrared divergency. Since one is interested in the coupling with color neutral states, this expression can equivalently be replaced by

$$
a\left(\underline{x}_{0}, \underline{x}_{1}, \underline{x}_{0^{\prime}}, \underline{x}_{1^{\prime}}\right)=2 \pi^{2} \ln \left|\frac{\underline{x}_{00^{\prime}} \underline{x}_{11^{\prime}}}{\underline{x}_{01^{\prime}} \underline{x}_{0^{\prime} 1}}\right| \ln \left|\frac{\underline{x}_{00^{\prime}} \underline{x}_{11^{\prime}}}{\underline{x}_{01} \underline{x}_{0^{\prime} 1^{\prime}}}\right|
$$

because of the conservation of color current [41]. In the case of the dipole model, instead of labeling the gluons, one labels the dipoles. Thus, the contributions of the figure 5 should be equal to the contribution of figure 6 plus permutations $\left(\underline{x}_{0} \leftrightarrow \underline{x}_{1}\right)$ and $\left(\underline{x}_{0^{\prime}} \leftrightarrow \underline{x}_{1^{\prime}}\right)$ (except for normalisation factors). Indeed, one can check that

$$
\begin{aligned}
& \sigma_{D D}\left(\underline{x}, \underline{x}^{\prime}, \underline{b}-\underline{b}^{\prime}\right)=\frac{\alpha_{s}}{(2 \pi)^{2}}\left[a\left(\underline{x}_{0}, \underline{x}_{1}, \underline{x}_{0^{\prime}}, \underline{x}_{1^{\prime}}\right)+\left(\underline{x}_{0} \leftrightarrow \underline{x}_{1}\right)+\left(\underline{x}_{0^{\prime}} \leftrightarrow \underline{x}_{1^{\prime}}\right)+\left(\underline{x}_{0} \leftrightarrow \underline{x}_{1}, \underline{x}_{0^{\prime}} \leftrightarrow \underline{x}_{1^{\prime}}\right)\right] \\
& =2 \alpha_{s}\left[a\left(\underline{x}_{0}, \underline{x}_{1}, \underline{x}_{0^{\prime}}, \underline{x}_{1^{\prime}}\right)+\left(\underline{x}_{0} \leftrightarrow \underline{x}_{1}\right)\right] .
\end{aligned}
$$


Using the expression (26) of Ref. 29 in the Born approximation, that is making $g=0$, one finally obtains

$$
\begin{aligned}
& \sigma_{D D}\left(\underline{x}, \underline{x}^{\prime}, \underline{b}-\underline{b}^{\prime}\right)=\frac{2 \alpha_{s}^{2}}{(2 \pi)^{2}} \sum_{n=-\infty}^{+\infty} \int_{-\infty}^{+\infty} d \nu \int d^{2} \underline{w}\left(\nu^{2}+\frac{n^{2}}{4}\right) \frac{1+(-1)^{n}}{\left(\nu^{2}+\left(\frac{n-1}{2}\right)^{2}\right)\left(\nu^{2}+\left(\frac{n+1}{2}\right)^{2}\right)} \\
& \times E^{n, \nu *}\left(\underline{b}+\frac{\underline{x}}{2}-\underline{w}, \underline{b}-\frac{\underline{x}}{2}-\underline{w}\right) E^{n, \nu}\left(\underline{b}^{\prime}+\frac{\underline{x}^{\prime}}{2}-\underline{w}, \underline{\underline{x}^{\prime}}-\frac{\underline{x}^{\prime}}{2}-\underline{w}\right),
\end{aligned}
$$

since

$$
E^{n, \nu}\left(x_{b 0}, x_{a 0}\right)=(-1)^{n} E^{n, \nu}\left(x_{a 0}, x_{b 0}\right) .
$$

One can check on this expression that when integrating over $\underline{b}^{\prime}-\underline{b}$ and averaging over angles, one recovers the total cross-section (A.14). Indeed, using the mixed representation (2.21), one obtains

$$
\begin{aligned}
\sigma_{D D}\left(\underline{x}, \underline{x}^{\prime}, \underline{b}-\underline{b}^{\prime}\right) & =2 \alpha_{s}^{2} \int \frac{d^{2} \underline{q}}{(2 \pi)^{2}} e^{-i \underline{q} \cdot\left(\underline{b}^{\prime}-\underline{b}\right)} \frac{x x^{\prime}}{16} \sum_{n=-\infty}^{+\infty} \int_{-\infty}^{+\infty} d \nu \frac{1+(-1)^{n}}{\left(\nu^{2}+\left(\frac{n-1}{2}\right)^{2}\right)\left(\nu^{2}+\left(\frac{n+1}{2}\right)^{2}\right)} \\
& \times E_{q}^{n, \nu *}\left(x^{\prime}\right) E_{q}^{n, \nu}(x) .
\end{aligned}
$$

Thus,

$$
\begin{aligned}
\sigma_{D D}\left(x, x^{\prime}\right) & =\int d^{2}\left(\underline{b}^{\prime}-\underline{b}\right) \sigma_{D D}\left(\underline{x}, \underline{x}^{\prime}, \underline{b}-\underline{b}^{\prime}\right) \\
& =2 \alpha_{s}^{2} \frac{x x^{\prime}}{16} \sum_{n=-\infty}^{+\infty} \int_{-\infty}^{+\infty} d \nu \frac{1+(-1)^{n}}{\left(\nu^{2}+\left(\frac{n-1}{2}\right)^{2}\right)\left(\nu^{2}+\left(\frac{n-1}{2}\right)^{2}\right)} \lim _{q \rightarrow 0}\left[E_{q}^{n, \nu *}\left(x^{\prime}\right) E_{q}^{n, \nu}(x)\right] .
\end{aligned}
$$

Using the expansion (A.1) of Ref. [29], one obtains

$$
\begin{aligned}
& \left.E_{q}^{n, \nu *}\left(x^{\prime}\right) E_{q}^{n, \nu}(x)\right|_{q \ll 1 / x, 1 / x^{\prime}} \\
& =\left(\frac{x x^{\prime *}}{x^{*} x^{\prime}}\right)^{n / 2}\left|\frac{x}{x^{\prime}}\right|^{-2 i \nu}\left[1+\left(\frac{q x^{*}}{q^{*} x}\right)^{n}|q x|^{4 i \nu} e^{i \delta(n, \nu)}\right]\left[1+\left(\frac{q^{*} x^{\prime}}{q x^{\prime *}}\right)^{n}\left|q x^{\prime}\right|^{-4 i \nu} e^{-i \delta(n, \nu)}\right] \\
& =\left(\frac{x x^{*}}{x^{*} x^{\prime}}\right)^{n / 2}\left|\frac{x}{x^{\prime}}\right|^{-2 i \nu}\left[1+\left(\frac{x^{\prime} x^{*}}{x x^{\prime *}}\right)^{n}\left|\frac{x}{x^{\prime}}\right|^{4 i \nu}+\left(\frac{q x^{*}}{q^{*} x}\right)^{n}|q x|^{4 i \nu} e^{i \delta(n, \nu)}+\left(\frac{q^{*} x^{\prime}}{q x^{\prime *}}\right)^{n}\left|q x^{\prime}\right|^{-4 i \nu} e^{-i \delta(n, \nu)}\right]
\end{aligned}
$$

where $e^{i \delta(n, \nu)}$ is a phase given by Eq. (A.2) of Ref. [29]. When $q \rightarrow 0$, only the two first terms remain. Due to the symetry of $\frac{1+(-1)^{n}}{\left(\nu^{2}+\left(\frac{n-1}{2}\right)^{2}\right)\left(\nu^{2}+\left(\frac{n-1}{2}\right)^{2}\right)}$ when $\nu \rightarrow-\nu$ or $n \rightarrow-n$, the contribution of these two terms is the same when summing over $n$ and integrating over $\nu$. Thus,

$$
\sigma_{D D}\left(x, x^{\prime}\right)=4 \alpha_{s}^{2} \frac{|x|\left|x^{\prime}\right|}{16} \sum_{n=-\infty}^{+\infty} \int_{-\infty}^{+\infty} d \nu \frac{1+(-1)^{n}}{\left(\nu^{2}+\left(\frac{n-1}{2}\right)^{2}\right)\left(\nu^{2}+\left(\frac{n+1}{2}\right)^{2}\right)}\left(\frac{x x^{*}}{x^{*} x^{\prime}}\right)^{n / 2}\left|\frac{x}{x^{\prime}}\right|^{-2 i \nu} .
$$

Averaging over the angles, the only remaining term is $n=0$. Thus,

$$
\sigma_{D D}\left(x, x^{\prime}\right)=\frac{\alpha_{s}^{2}}{2} \int_{-\infty}^{+\infty} d \nu|x|^{1+2 i \nu}\left|x^{\prime}\right|^{1-2 i \nu} \frac{1}{\left(\nu^{2}+\frac{1}{4}\right)^{2}}
$$

which is identical to Eq. (A.13). 


\section{A.3 Calculation of $\hat{\sigma}_{g d}$ using eikonal methods}

In this section we compute the elementary Born cross-section $\hat{\sigma}_{\gamma d} / k^{2}$ of the process

$$
d(\underline{x}) g(k) \rightarrow d(\underline{x})
$$

for a dipole of transverse size $\underline{x}$ and a soft gluon of virtuality $\underline{k}^{2}$, in light-cone gauge. This process is illustrated in figure 7 . Consider a dipole of transverse size $\underline{x}=\underline{x}_{a}-\underline{x}_{b}$. The corresponding

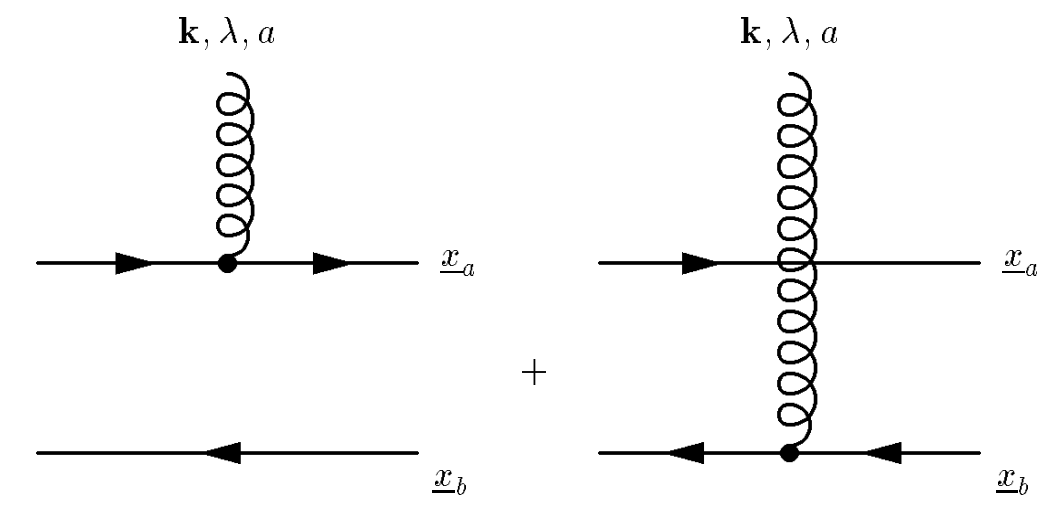

Figure 7: Amplitudes contributing to the elementary dipole-gluon cross-section.

eikonal current has the same expression than in covariant gauge (A.3). This would not be the case for a more complicate system for which the current would be rotated in color space (see for example Ref.42]). In light-cone perturbation theory, $k^{2}=2 k^{+} k^{-}-\underline{k}^{2}=0$. The corresponding current then reads

$$
j^{a+}(k)=-i g T^{a} \frac{2 k^{+}}{\underline{k}^{2}}\left(e^{-i \underline{x}_{a} \cdot \underline{k}}-e^{-i \underline{x}_{b} \cdot \underline{k}}\right) .
$$

The Born cross-section then reads, summing over color and polarization of the emitted gluon and averaging over the color of the dipole,

$$
\begin{aligned}
\frac{\hat{\sigma}_{g d}}{\underline{k}^{2}} & =\frac{1}{2(2 \pi)^{2} N_{c}} \operatorname{Tr}\left|j^{+} \epsilon^{-\lambda}\right|^{2} \\
& =\frac{1}{2(2 \pi)^{2} N_{c}} \sum_{\lambda=1,2} \sum_{a} g^{2} \operatorname{Tr}\left(T^{a} T^{a}\right)\left(e^{-i \underline{k}} \cdot \underline{x}_{a}-e^{-i \underline{k}} \cdot \underline{x}_{b}\right)\left(\left(e^{i \underline{k} \cdot \underline{x}_{a}}-e^{i \underline{k} \cdot \underline{x}_{b}}\right) 4\left(\frac{\underline{k} \cdot \underline{\epsilon}^{\lambda}}{\underline{k}^{2}}\right)^{2}\right.
\end{aligned}
$$

Here one takes into account the fact that the dominant polarization of the soft emitted gluon in light-cone gauge corresponds to

$$
\epsilon_{\mu}^{\lambda}=\left(\stackrel{+}{0}, \frac{\underline{k} \cdot \underline{\epsilon}^{\lambda}}{k_{+}}, \underline{\perp}^{\lambda}\right) \sim\left(\stackrel{+}{0}, \frac{\underline{k} \cdot \underline{\epsilon}^{\lambda}}{k_{+}}, \stackrel{\perp}{0}\right)
$$

in the soft limit. This finally yields

$$
\frac{\hat{\sigma}_{g d}}{\underline{k}^{2}}=\frac{g^{2} C_{F}}{2(2 \pi)^{2}}\left(2-e^{i \underline{k} \cdot \underline{x}}-e^{-i \underline{k} \cdot \underline{x}}\right) \frac{4}{\underline{k}^{2}}=\frac{\alpha_{s} N_{c}}{\pi}\left(2-e^{i \underline{k} \cdot \underline{x}}-e^{-i \underline{k} \cdot \underline{x}}\right) \frac{1}{\underline{k}^{2}} .
$$


Let us make two comments. First, the dominant polarization of the exchanged gluon is longitudinal, due to the expression (A.38). However, the polarization tensor in light-cone gauge reads

$$
d^{\mu \nu}=g^{\mu \nu}-\frac{\eta^{\mu} k^{\nu}+\eta^{\nu} k^{\mu}}{\eta \cdot k} .
$$

In the Regge limit, since $j_{\nu}$ is proportionnal to $\bar{\eta}$, the relevant component of this tensor is $d^{\mu-}$, and since $\epsilon^{+}=0$ in light cone gauge, it turns out that $\epsilon_{\mu} d^{\mu-}=\epsilon^{i} d^{i-}$, and thus the exchanged gluon has a physical polarization, as it is expected in high-energy factorization.

Secondly, when integrating this cross-section over $k$, one can recover the dipole emission kernel which was constructed in Ref. [14].

\section{A.4 Calculation of the onium-onium cross section in the laboratory frame of one onium}

In this appendix we illustrate the frame invariance of the onium-onium cross section by an explicit calculation in the laboratory frame where the left-moving heavy quark-antiquark pair is close to be at rest. Let us first consider as in section 2.2 the scattering of two heavy quarkantiquark pairs of transverse sizes $\underline{x}_{1}$ and $\underline{x}_{2}$ which scatter through the exchange of a pair of gluons between two elementary excited dipoles of transverse sizes $\underline{x}_{1}^{\prime}$ and $\underline{x}_{1}^{\prime}$. The corresponding expression for the scattering amplitude $F^{(1)}$ is displayed in formula (2.47). Using the same trick which led us to formula (2.50), we integrate over $d^{2} \underline{b}_{i n t} d^{2} \underline{x}_{1}^{\prime}$ and $d^{2} \underline{w}_{2}$, which yields

$$
\begin{aligned}
& F^{(1)}\left(\underline{x}_{1}, \underline{x}_{2}, \tilde{Y}, \underline{b}\right)=-2 \frac{\alpha_{s}^{2}(16)^{2}}{(2 \pi)^{6}} \frac{\pi^{4}}{2} \sum_{n_{1}=-\infty}^{+\infty} \sum_{n=-\infty}^{+\infty} \int_{-\infty}^{+\infty} \frac{d \nu_{1}}{(2 \pi)^{3}} \int_{-\infty}^{+\infty} d \nu \int \frac{d^{2} \underline{w}_{1}}{x_{1}^{\prime 2}} \\
& \times \int d^{2} \underline{w} \int \frac{d^{2} \underline{x}_{1}^{\prime}}{2 \pi x_{1}^{\prime 2}} \int d^{2} \underline{b}_{1}\left(\nu_{1}^{2}+\frac{n_{1}^{2}}{4}\right) \\
& \times\left(\nu^{2}+\frac{n^{2}}{4}\right) \frac{1+(-1)^{n}}{\left(\nu^{2}+\left(\frac{n-1}{2}\right)^{2}\right)\left(\nu^{2}+\left(\frac{n+1}{2}\right)^{2}\right)} \exp \left(\frac{2 \alpha_{s} N_{c}}{\pi}\left(\chi\left(n_{1}, \nu_{1}\right) \tilde{Y}_{1}+\chi(n, \nu) \tilde{Y}_{2}\right)\right) \\
& \times E^{n_{1}, \nu_{1}}\left(\underline{b}_{1}+\frac{\underline{x}_{1}^{\prime}}{2}-\underline{w}_{1}, \underline{b}_{1}-\frac{\underline{x}_{1}^{\prime}}{2}-\underline{w}_{1}\right) E^{n_{1}, \nu_{1} *}\left(\frac{\underline{x}_{1}}{2}-\underline{w}_{1},-\frac{\underline{x}_{1}}{2}-\underline{w}_{1}\right) \\
& \times E^{n, \nu *}\left(\underline{b}_{1}+\frac{\underline{x}_{1}^{\prime}}{2}-\underline{w}, \underline{b}_{1}-\frac{\underline{x}_{1}^{\prime}}{2}-\underline{w}\right) E^{n, \nu}\left(\frac{\underline{x}_{2}}{2}-\underline{w}+\underline{b}, \frac{-\underline{x}_{2}}{2}-\underline{w}+\underline{b}\right)
\end{aligned}
$$

Integrating over $d^{2} \underline{x}_{1}^{\prime} d^{2} \underline{b}_{1}$ and $d^{2} \underline{w}$ would give the result (2.54). It would show in particular that in the previous formula $n_{1}$ can be replaced by $n$ and $\nu_{1}$ by $\nu$. Since $\tilde{Y}=\tilde{Y}_{1}+\tilde{Y}_{2}$, the previous formula can be written as

$$
F^{(1)}\left(\underline{x}_{1}, \underline{x}_{2}, \tilde{Y}, \underline{b}\right)=-\frac{1}{2} \int d^{2} \underline{b}_{1} \int \frac{d^{2} \underline{x}_{1}^{\prime}}{2 \pi x_{1}^{\prime 2}} n\left(\underline{x}_{1}, \underline{x}_{1}^{\prime}, \tilde{Y}, \underline{b}_{1}\right) \sigma_{D D}\left(\underline{x}_{1}^{\prime}, \underline{x}_{2}, \underline{b}_{1}-\underline{b}\right),
$$

which is exactly what one would obtain when computing this process in the boosted frame where the left-moving onium is close to be at rest. Indeed, let us consider the dipole distribution inside the left-moving onium in its laboratory frame, namely $n\left(\underline{x}_{2}, \underline{x}_{2}^{\prime}, \tilde{Y}=0, \underline{b}_{2}\right)$. Using expression (2.19), it reads, using the completeness condition for the functions $E^{n, \nu}$ (see equation (25) of 
Ref. [29))

$$
\begin{aligned}
& n\left(\underline{x}_{2}, \underline{x}_{2}^{\prime}, \tilde{Y}=0, \underline{b}_{2}\right) \\
& =\sum_{n=-\infty}^{+\infty} 16 \int \frac{d \nu}{(2 \pi)^{3}} \frac{d^{2} w}{x_{2}^{\prime 2}}\left(\nu^{2}+\frac{n^{2}}{4}\right) E^{n, \nu}\left(\underline{b}_{2}+\frac{\underline{x}_{2}^{\prime}}{2}-\underline{w}, \underline{b}-\frac{\underline{x}_{2}^{\prime}}{2}-\underline{w}\right) E^{n, \nu *}\left(\frac{\underline{x}_{2}}{2}-\underline{w},-\frac{\underline{x}_{2}}{2}-\underline{w}\right) \\
& =\frac{(2 \pi)^{4}}{(2 \pi)^{3}} x_{2}^{2} \delta^{2}\left(\underline{b}_{2}+\frac{\underline{x}_{2}^{\prime}-\underline{x}_{2}}{2}\right) \delta^{2}\left(-\underline{b}_{2}+\frac{\underline{x}_{2}^{\prime}-\underline{x}_{2}}{2}\right) \\
& =2 \pi x_{2}^{2} \delta^{2}\left(\underline{b}_{2}\right) \delta^{2}\left(\underline{x}_{2}^{\prime}-\underline{x}_{2}\right) .
\end{aligned}
$$

Calculating the scattering amplitude $F^{(1)}$ with formula 2.7$)$ in this laboratory frame, one gets

$$
\begin{aligned}
& F^{(1)}\left(x_{1}, x_{2}, b, \tilde{Y}\right)=-\frac{1}{2} \int_{0}^{\infty} \frac{d^{2} \underline{x}_{1}^{\prime}}{2 \pi x_{1}^{\prime 2}} \frac{d^{2} \underline{x}_{2}^{\prime}}{2 \pi x_{2}^{\prime 2}} d^{2} \underline{b}_{1} d^{2} \underline{b}_{2} d^{2}\left(\underline{b}_{2}^{\prime}-\underline{b}_{1}^{\prime}\right) \delta^{2}\left(\underline{b}_{1}-\underline{b}_{2}-\underline{b}_{1}^{\prime}+\underline{b}_{2}^{\prime}-\underline{b}\right) \\
& \times n\left(\underline{x}_{1}, \underline{x}_{1}^{\prime}, \tilde{Y}, \underline{b}_{1}\right) 2 \pi x_{2}^{2} \delta^{2}\left(\underline{b}_{2}\right) \delta^{2}\left(\underline{x}_{2}^{\prime}-\underline{x}_{2}\right) \sigma_{D D}\left(\underline{x}_{1}^{\prime}, \underline{x}_{2}^{\prime}, \underline{b}_{1}^{\prime}-\underline{b}_{2}^{\prime}\right) \\
& =-\frac{1}{2} \int d^{2} \underline{b}_{1} \int \frac{d^{2} \underline{x}_{1}^{\prime}}{2 \pi x_{1}^{\prime 2}} n\left(\underline{x}_{1}, \underline{x}_{1}^{\prime}, \tilde{Y}, \underline{b}_{1}\right) \sigma_{D D}\left(\underline{x}_{1}^{\prime}, \underline{x}_{2}, \underline{b}_{1}-\underline{b}\right),
\end{aligned}
$$

where we have performed in the last step the integration with respect to $d^{2} \underline{x}_{2}^{\prime} d^{2} \underline{b}_{2} d^{2}\left(\underline{b}_{2}^{\prime}-\underline{b}_{1}^{\prime}\right)$. This result is identical to Eq. (A.42).

\section{A.5 Approximate calculation of $F^{(1)}$ in the asymptotic regime}

In this appendix we calculate the onium-onium scattering amplitude at fixed impact parameter in the asymptotic regime. In that case the distributions of dipoles $n\left(\underline{x}_{i}, \underline{x}_{i}^{\prime}, \tilde{Y}_{i}, \underline{b}_{i}\right)$ can be approximated by keeping only the contribution $n=0$ to formula (2.23). We show that even in this limit it is important to consider the exact expression (A.23) of the elementary dipoledipole cross-section. In Ref. [15], the onium-onium cross-section at fixed impact parameter was computed considering the interaction of two elementary dipoles of transverse size $\underline{x}_{1}^{\prime}$ and $\underline{x}_{1}^{\prime}$, situated at the same point $\underline{b}_{1}^{\prime}=\underline{b}_{2}^{\prime}$, interacting through the elementary total cross-section $\sigma_{D D}\left(x_{1}^{\prime}, x_{2}^{\prime}\right)$, and the dipole distributions were averaged over angles. Indeed, the elementary cross-section at fixed impact parameter $\sigma_{D D}\left(\underline{x}_{1}^{\prime}, \underline{x}_{2}^{\prime}, \underline{b}_{1}^{\prime}-\underline{b}_{2}^{\prime}\right)$ is dominated by configurations $\underline{b}_{1}^{\prime} \sim \underline{b}_{2}^{\prime}$, since $\sigma_{D D}\left(\underline{x}_{1}^{\prime}, \underline{x}_{2}^{\prime}, \underline{b}_{1}^{\prime}-\underline{b}_{2}^{\prime}\right) \sim \frac{1}{\left|\underline{b}_{1}^{\prime}-\underline{b}_{2}^{\prime}\right|^{4}}$ when $\left|\underline{b}_{1}^{\prime}-\underline{b}_{2}^{\prime}\right|$ is large (this can be seen by expanding formula (A.23) ). Thus, it is possible in first approximation to make $\underline{b}_{1}^{\prime}=\underline{b}_{2}^{\prime}$ and to replace $\sigma_{D D}\left(\underline{x}_{1}^{\prime}, \underline{x}_{2}^{\prime}, \underline{b}_{1}^{\prime}-\underline{b}_{2}^{\prime}\right)$ by its integral over $\underline{b}_{1}^{\prime}-\underline{b}_{2}^{\prime}$, which is equal to $\sigma_{D D}\left(x_{1}^{\prime}, x_{2}^{\prime}\right)$ when averaging over angles (see appendix A.2). Note that in the asymptotic regime where it is possible to keep only the contributions $n_{1}=n_{2}=0$ in the dipole distributions, the elementary cross-section will only contribute through the quantum number $n=0$, since this quantum number is conserved (see Eqs. (2.47-2.54) for example), and thus the averaging over angles in the elementary crosssection is automatically implied (see Eqs. (A.33-A.34). However, we will see below that the approximation of integrating the elementary dipole-dipole cross section over impact parameter is exact only when calculating the total cross-section.

We thus calculate first the cross-section at fixed impact parameter in these approximations and compare the result to the exact formula (2.59). Let us consider two heavy quark-antiquark pairs of transverse size $\underline{x}_{1}$ and $\underline{x}_{2}$, which scatter through the exchange of a pair of gluons between two elementary dipoles, respectively of transverse size $\underline{x}_{1}^{\prime}$ and $\underline{x}_{2}^{\prime}$, and at a distance 
from the center of the quark-antiquark pair $\underline{b}_{1}$ and $\underline{b}_{2}$. In this approximation, the corresponding scattering amplitude $F^{(1)}$ given in Eq. (2.7) simplifies in

$$
F^{(1)}\left(\underline{x}_{1}, \underline{x}_{2}, \tilde{Y}, \underline{b}\right)=-\frac{1}{2} \int_{0}^{\infty} \frac{d x_{1}^{\prime}}{x_{1}^{\prime}} \frac{d x_{2}^{\prime}}{x_{2}^{\prime}} d^{2} \underline{b}_{1} n\left(\underline{x}_{1}, \underline{x}_{1}^{\prime}, \underline{b}_{1}, \tilde{Y}_{1}\right) n\left(\underline{x}_{2}, \underline{x}_{2}^{\prime}, \underline{b}-\underline{b}_{1}, \tilde{Y}_{2}\right) \sigma_{D D}\left(x_{1}^{\prime}, x_{2}^{\prime}\right) .
$$

The rapidity $\tilde{Y}_{1}$ and $\tilde{Y}_{2}$ are such that $\tilde{Y}=\tilde{Y}_{1}+\tilde{Y}_{2} \cdot F^{(1)}$ involves the elementary dipole-dipole total cross-section, which has been evaluated in [15], and which is calculated in appendix A.1] using eikonal techniques. For two dipoles of transverse size $\underline{x}_{1}^{\prime}$ and $\underline{x}_{2}^{\prime}$, the elementary forward dipole-dipole cross-section reads (see Eq. (A.11))

$$
\sigma_{D D}\left(x_{1}^{\prime}, x_{2}^{\prime}\right)=2 \pi \alpha_{s}^{2} x_{<}^{2}\left[1+\ln \frac{x_{>}}{x_{<}}\right],
$$

where $x_{<}=\operatorname{Min}\left(x_{1}^{\prime}, x_{2}^{\prime}\right)$ et $x_{>}=\operatorname{Max}\left(x_{1}^{\prime}, x_{2}^{\prime}\right)$.

We write $F^{(1)}$ given by Eq. (A.45) as

$$
F^{(1)}\left(x_{1}, x_{2}, b, \tilde{Y}\right)=\int_{-\infty}^{\infty} \frac{d \nu}{2 \pi} F_{\nu}^{(1)}\left(x_{1}, x_{2}, b\right) .
$$

Using the expression (A.14) of the elementary dipole-dipole cross section $\sigma_{D D}, F_{\nu}^{(1)}$ then reads

$$
\begin{aligned}
& F_{\nu}^{(1)}=-\frac{\pi \alpha_{s}^{2}}{2} \frac{1}{\left(\nu^{2}+\frac{1}{4}\right)^{2}} \int_{-\infty}^{\infty} \frac{d \nu_{1}}{2 \pi} \int_{-\infty}^{\infty} \frac{d \nu_{2}}{2 \pi} \exp \left(\frac{2 \alpha_{s} N_{c}}{\pi}\left(\chi\left(0, \nu_{1}\right) \tilde{Y}_{1}+\chi\left(0, \nu_{2}\right) \tilde{Y}_{2}\right)\right) \\
& \times \int \frac{d^{2} \underline{x}_{1}^{\prime}}{2 \pi x_{1}^{2^{\prime}}} \int \frac{d^{2} \underline{x}_{2}^{\prime}}{2 \pi x_{2}^{\prime 2}} \int d^{2} \underline{b}_{1} \int d^{2} \underline{b}_{2} \delta^{2}\left(\underline{b}-\underline{b_{1}}-\underline{b_{2}}\right) n_{\nu_{1}}\left(x_{1}, x_{1}^{\prime}, \underline{b}_{1}\right) n_{\nu_{2}}\left(x_{2}, x_{2}^{\prime}, \underline{b}_{2}\right)\left(x_{1}^{\prime}\right)^{1+2 i \nu}\left(x_{2}^{\prime}\right)^{1-2 i \nu} .
\end{aligned}
$$

Using the representation

$$
\delta^{2}\left(\underline{b}-\underline{b}_{1}-\underline{b}_{2}\right)=\int \frac{d^{2} \underline{q}}{(2 \pi)^{2}} \exp \left(-i \underline{q} \cdot\left(\underline{b}-\underline{b}_{1}-\underline{b}_{2}\right),\right.
$$

and the expression $(2.23)$ of $n\left(\underline{x}, \underline{x}^{\prime}, \tilde{Y}, \underline{b}\right)$, one gets

$$
\begin{aligned}
& F_{\nu}^{(1)}=-\frac{\pi \alpha_{s}^{2}}{2} \frac{1}{\left(\nu^{2}+\frac{1}{4}\right)^{2}} \int \frac{d^{2} \underline{q}}{(2 \pi)^{2}} \int_{-\infty}^{\infty} \frac{d \nu_{1}}{2 \pi} \int_{-\infty}^{\infty} \frac{d \nu_{2}}{2 \pi} \exp \left(\frac{2 \alpha_{s} N_{c}}{\pi}\left(\chi\left(0, \nu_{1}\right) \tilde{Y}_{1}+\chi\left(0, \nu_{2}\right) \tilde{Y}_{2}\right)\right) \\
& \times \int \frac{d^{2} \underline{x}_{1}^{\prime}}{2 \pi x_{1}^{\prime 2}} \int \frac{d^{2} \underline{x}_{2}^{\prime}}{2 \pi x_{2}^{\prime 2}} e^{-i \underline{q} \cdot \underline{b}} \frac{x_{1}}{x_{1}^{\prime}} E_{q}^{0, \nu_{1} *}\left(x_{1}\right) E_{q}^{0, \nu_{1}}\left(x_{1}^{\prime}\right) \frac{x_{2}}{x_{2}^{\prime}} E_{q}^{0, \nu_{2} *}\left(x_{2}\right) E_{q}^{0, \nu_{2}}\left(x_{2}^{\prime}\right)\left(x_{1}^{\prime}\right)^{1+2 i \nu}\left(x_{2}^{\prime}\right)^{1-2 i \nu}
\end{aligned}
$$

Let us compute the integrations with respect to $x_{i}^{\prime}$. Defining

$$
f_{\nu_{i}}(\nu, q)=\int \frac{d^{d} \underline{x}_{i}^{\prime}}{2 \pi} E_{q}^{0, \nu_{i}}\left(x_{i}^{\prime}\right)^{-2+2 i \epsilon_{i} \nu}
$$

where $\epsilon_{1}=1$ and $\epsilon_{2}=-1$, this function reads, taking into account the representation (2.29) and introducing a dimensional regularization $d=2+2 \epsilon$ because of the divergency when $x_{i}^{\prime} \rightarrow 0$,

$$
\begin{aligned}
& f_{\nu_{i}}(\nu, q)=\frac{4 \pi^{3}}{b_{0, \nu_{i}}}\left(\frac{q}{2}\right)^{2 i \nu_{i}} \frac{1}{\Gamma^{2}\left(\frac{1}{2}+i \nu_{i}\right)} \int_{0}^{1} d \alpha_{i}\left(\alpha_{i}\left(1-\alpha_{i}\right)\right)^{-\frac{1}{2}} \\
& \times \frac{\pi^{\epsilon}}{\Gamma(1+\epsilon)} \int_{0}^{+\infty} d x_{i}^{\prime}\left(x_{i}^{\prime}\right)^{-1+2 i \epsilon_{i} \nu+2 \epsilon} K_{-2 i \nu_{i}}\left(q x_{i}^{\prime} \sqrt{\alpha_{i}\left(1-\alpha_{i}\right)}\right) J_{0}\left(q \frac{x_{i}^{\prime}}{2}\left(1-2 \alpha_{i}\right)\right),
\end{aligned}
$$


where we have performed the integration with respect to the angle $\left(\underline{q}, \underline{x}_{i}^{\prime}\right)$. One can now perform the integration with respect to $x_{i}^{\prime}$, using the formula (6.576) of Ref. [33]:

$$
\begin{aligned}
& \int_{0}^{\infty} x^{-\lambda} K_{\rho}(a x) J_{\sigma}(b x) d x=\frac{1}{4}\left(\frac{b}{a}\right)^{\sigma}\left(\frac{a}{2}\right)^{\lambda-1} \frac{\Gamma\left(\frac{\sigma-\lambda+1+\rho}{2}\right) \Gamma\left(\frac{\sigma-\lambda+1-\rho}{2}\right)}{\Gamma(\sigma+1)} \\
& \times_{2} F_{1}\left(\frac{\sigma-\lambda+1+\rho}{2}, \frac{\sigma-\lambda+1-\rho}{2} ; \sigma+1 ;-\frac{b^{2}}{a^{2}}\right) .
\end{aligned}
$$

Here $\sigma=0, \rho=-2 i \nu_{i}, b=\frac{q}{2}\left(1-2 \alpha_{i}\right), a=q \sqrt{\alpha_{i}\left(1-\alpha_{i}\right)}$ and $-\lambda=2 i \epsilon_{i} \nu-1+2 \epsilon$. Thus,

$$
\begin{aligned}
& f_{\nu_{i}}(\nu, q)=\frac{4 \pi^{3}}{b_{0, \nu_{i}}} \frac{1}{\Gamma^{2}\left(\frac{1}{2}+i \nu_{i}\right)} \frac{\pi^{\epsilon}}{\Gamma(1+\epsilon)} \frac{1}{4} \int_{0}^{1} d \alpha_{i}\left(\alpha_{i}\left(1-\alpha_{i}\right)\right)^{-\frac{1}{2}-i \epsilon_{i} \nu-\epsilon}\left(\frac{q}{2}\right)^{2 i \nu_{i}-2 i \epsilon_{i} \nu-2 \epsilon} \\
& \times \Gamma\left(i \epsilon_{i} \nu-i \nu_{i}+\epsilon\right) \Gamma\left(i \epsilon_{i} \nu+i \nu_{i}+\epsilon\right){ }_{2} F_{1}\left(i \epsilon_{i} \nu-i \nu_{i}+\epsilon, i \epsilon_{i} \nu+i \nu_{i}+\epsilon ; 1 ;-\frac{1}{4} \frac{\left(1-2 \alpha_{i}\right)^{2}}{\alpha_{i}\left(1-\alpha_{i}\right)}\right) .
\end{aligned}
$$

One can now perform the integration with respect to $\nu_{i}$. We thus define

$$
g^{i}\left(\nu, x_{i}, q\right)=\int_{-\infty}^{+\infty} \frac{d \nu_{i}}{2 \pi} E_{q}^{0, \nu_{i} *}\left(x_{i}\right) f_{\nu_{i}}(\nu, q) \exp \left(\frac{2 \alpha_{s} N_{c}}{\pi} \chi\left(0, \nu_{i}\right) \tilde{Y}_{i}\right)
$$

The analytic structure of the integrand is very simple: it has poles at $\nu_{i}= \pm\left(\epsilon_{i} \nu-i \epsilon\right)$. The integration contour can be closed either in the upper plane or in the lower plane, and give the same contribution. For $g^{1}$ we close the contour in the lower plane so as to pick up the residue at $\nu_{1}=\nu$, and for $g^{2}$ we close the contour in the lower plane so as to pick up the residue at $\nu_{2}=-\nu$. Since

$$
{ }_{2} F_{1}\left(0,2 i \nu ; 1 ;-\frac{1}{4} \frac{\left(1-2 \alpha_{1}\right)^{2}}{\alpha_{1}\left(1-\alpha_{1}\right)}\right)={ }_{2} F_{1}\left(-2 i \nu, 0 ; 1 ;-\frac{1}{4} \frac{\left(1-2 \alpha_{2}\right)^{2}}{\alpha_{2}\left(1-\alpha_{2}\right)}\right)=1,
$$

one gets

$g^{i}\left(\nu, x_{i}, q\right)=\frac{\pi^{3}}{b_{0, \epsilon_{i} \nu}} \frac{\Gamma\left(2 i \epsilon_{i} \nu\right)}{\Gamma^{2}\left(\frac{1}{2}+i \epsilon_{i} \nu\right)} \int_{0}^{1} d \alpha_{i}\left(\alpha_{i}\left(1-\alpha_{i}\right)\right)^{-\frac{1}{2}-i \epsilon_{i} \nu} E_{q}^{0, \epsilon_{i} \nu *}\left(x_{i}\right) \exp \left(\frac{2 \alpha_{s} N_{c}}{\pi} \chi(0, \nu) \tilde{Y}_{i}\right)$,

since $\chi(0, \nu)$ is an even function of $\nu$. Performing the integration with respect to $\alpha_{i}$

$$
\int_{0}^{1} d \alpha_{i}\left(\alpha_{i}\left(1-\alpha_{i}\right)\right)^{-\frac{1}{2}-i \epsilon_{i} \nu}=\frac{\Gamma^{2}\left(-i \epsilon_{i} \nu+\frac{1}{2}\right)}{\Gamma\left(-2 i \epsilon_{i} \nu+1\right)}
$$

$F_{\nu}^{(1)}$ now reads

$$
\begin{aligned}
F_{\nu}^{(1)}= & -\frac{\pi \alpha_{s}^{2}}{2} \frac{x_{1} x_{2}}{\left(\nu^{2}+\frac{1}{4}\right)^{2}} \int \frac{d^{2} \underline{q}}{(2 \pi)^{2}} e^{-i \underline{q} \cdot \underline{b}} g^{1}\left(\nu, x_{1}, q\right) g^{2}\left(\nu, x_{2}, q\right) \\
=- & -\frac{\pi \alpha_{s}^{2}}{2} \frac{x_{1} x_{2}}{\left(\nu^{2}+\frac{1}{4}\right)^{2}} \int \frac{d^{2} \underline{q}}{(2 \pi)^{2}} e^{-i \underline{q} \cdot \underline{b}} \frac{\pi^{6}}{b_{0, \nu} b_{0,-\nu}} \frac{\Gamma(2 i \nu)}{\Gamma(1+2 i \nu)} \frac{\Gamma(-2 i \nu)}{\Gamma(1-2 i \nu)} \\
& \times E_{q}^{0, \nu *}\left(x_{1}\right) E_{q}^{0, \nu *}\left(x_{2}\right) \exp \left(\frac{2 \alpha_{s} N_{c}}{\pi} \chi(0, \nu) \tilde{Y}\right),
\end{aligned}
$$


when taking into account that $\tilde{Y}=\tilde{Y}_{1}+\tilde{Y}_{2}$. Note that formula A.59 could be equivalently obtained from Eq. (A.54) by performing the change of variable $\alpha_{i} \rightarrow z_{i}=\left(1-2 \alpha_{i}\right)^{2}$ and using the Watson theorem [43]. Taking the conjugate of formula (2.29) and then performing the change of variable $\alpha=\alpha^{\prime}-1$, one can verify that

$$
E_{q}^{0, \nu *}(\rho)=E_{-q}^{0,-\nu}(\rho)=E_{q}^{0,-\nu}(\rho) .
$$

Using formula (2.38), one finally gets

$$
F_{\nu}^{(1)}=-\frac{\pi \alpha_{s}^{2}}{8} \frac{x_{1} x_{2}}{\left(\nu^{2}+\frac{1}{4}\right)^{2}} \int \frac{d^{2} \underline{q}}{(2 \pi)^{2}} e^{-i \underline{q} \cdot \underline{b}} E_{q}^{0, \nu *}\left(x_{1}\right) E_{q}^{0, \nu}\left(x_{2}\right) \exp \left(\frac{2 \alpha_{s} N_{c}}{\pi} \chi(0, \nu) \tilde{Y}\right)
$$

that is

$$
F_{\nu}^{(1)}=-\frac{\pi \alpha_{s}^{2}}{8\left(\nu^{2}+\frac{1}{4}\right)^{2}} x_{2}^{2} n_{\nu}\left(x_{1}, x_{2}, b, \tilde{Y}\right)=-\frac{\pi \alpha_{s}^{2}}{8\left(\nu^{2}+\frac{1}{4}\right)^{2}} x_{1}^{2} n_{\nu}\left(x_{2}, x_{1}, b, \tilde{Y}\right) .
$$

which differs from the exact result (2.59) by a factor $\frac{1}{2}$. Thus, the approximation of using the elementary dipole-dipole total cross-section only gives half the correct result.

One expects that the previous approximations should be correct when calculating the total cross-section. Indeed, integrating (A.45) with respect to $\underline{b}$, one gets

$$
F^{(1)}\left(x_{1}, x_{2}, \tilde{Y}\right)=-\frac{1}{2} \int_{0}^{\infty} \frac{d x_{1}^{\prime}}{x_{1}^{\prime}} \frac{d x_{2}^{\prime}}{x_{2}^{\prime}} n\left(\underline{x}_{1}, \underline{x}_{1}^{\prime}, \tilde{Y}_{1}\right) n\left(\underline{x}_{2}, \underline{x}_{2}^{\prime}, \tilde{Y}_{2}\right) \sigma_{D D}\left(x_{1}^{\prime}, x_{2}^{\prime}\right) .
$$

From formulae (2.65) and (A.14), this yields

$$
\begin{aligned}
F^{(1)}\left(x_{1}, x_{2}, \tilde{Y}\right)= & -\frac{1}{2} \int_{-\infty}^{+\infty} d \nu \sum_{n_{1}=-\infty}^{+\infty} \int_{-\infty}^{+\infty} \frac{d \nu_{1}}{2 \pi} \sum_{n_{2}=-\infty}^{+\infty} \int_{-\infty}^{+\infty} \frac{d \nu_{2}}{2 \pi} \int \frac{d^{2} \underline{x}_{1}^{\prime}}{2 \pi x_{1}^{\prime 2}} \frac{d^{2} \underline{x}_{2}^{\prime}}{2 \pi x_{2}^{\prime 2}} \frac{x_{1}}{x_{1}^{\prime}}\left(\frac{x_{1}^{*} x_{1}^{\prime}}{x_{1} x_{1}^{\prime *}}\right)^{n_{1} / 2}\left|\frac{x_{1}^{\prime}}{x_{1}}\right|^{-2 i \nu_{1}} \frac{x_{2}}{x_{2}^{\prime}} \\
& \times \frac{\alpha_{s}^{2}}{2} \frac{1}{\left(\nu^{2}+\frac{1}{4}\right)^{2}}\left|x_{1}^{\prime}\right|^{1-2 i \nu}\left|x_{2}^{\prime}\right|^{1+2 i \nu} .
\end{aligned}
$$

Integrating with respect to $\underline{x}_{1}^{\prime}$ and $\underline{x}_{2}^{\prime}$ and using relation (2.67), the only remaining terms are $n_{1}=n_{2}=0$, due to the conservation of conformal weight, and one gets

$$
F^{(1)}\left(x_{1}, x_{2}, \tilde{Y}\right)=\frac{\pi \alpha_{s}^{2}}{2} \int \frac{d \nu}{2 \pi} \frac{1}{\left(\nu^{2}+\frac{1}{4}\right)^{2}}\left|x_{1}\right|^{1-2 i \nu}\left|x_{2}\right|^{1+2 i \nu} \exp \left(\frac{2 \alpha N_{c}}{\pi}\left(\chi\left(n_{1}, \nu_{1}\right) \tilde{Y}_{1}+\chi\left(n_{2}, \nu_{2}\right) \tilde{Y}_{2}\right)\right)
$$

that is

$$
F_{\{0, \nu\}}^{(1)}\left(x_{1}, x_{2}\right)=-\frac{\pi \alpha_{s}^{2} x_{2}^{2}}{4} \frac{1}{\left(\nu^{2}+\frac{1}{4}\right)^{2}} n_{\{0, \nu\}}\left(x_{1}, x_{2}\right)
$$

which is exactly the result (2.71).

\section{A.6 Expression of $E_{q}^{0, \nu}$ in terms of Bessel functions}

In this appendix we prove the expression (2.30) of $E_{q}^{0, \nu}$. Starting from equation (2.29) and making the change of variable $\alpha=\sin ^{2} \frac{t}{2}$, the mixed function $E_{q}^{0, \nu}$ reads

$$
E_{q}^{0, \nu}(\rho)=\frac{4 \pi^{3}}{b_{0, \nu}}\left(\frac{q}{2}\right)^{2 i \nu} \frac{1}{\Gamma^{2}\left(\frac{1}{2}+i \nu\right)} \int_{0}^{\pi} e^{i \frac{\underline{q} \cdot \underline{\rho}}{2} \cos t} K_{-2 i \nu}\left(\frac{q \rho}{2} \sin t\right) d t
$$


Let us calculate

$$
C=\int_{0}^{\frac{\pi}{2}} e^{i \frac{\underline{q} \cdot \underline{\rho}}{2} \cos t} K_{-2 i \nu}\left(\frac{q \rho}{2} \sin t\right) d t+\int_{\frac{\pi}{2}}^{\pi} e^{i \frac{q \underline{\underline{\rho}}}{2} \cos t} K_{-2 i \nu}\left(\frac{q \rho}{2} \sin t\right) d t .
$$

Making the change of variable $t^{\prime}=\pi-t$ in the second integral, one gets

$$
C=2 \int_{0}^{\frac{\pi}{2}} \cos \left(\frac{\underline{q} \cdot \underline{\rho}}{2} \cos t\right) K_{-2 i \nu}\left(\frac{q \rho}{2} \sin t\right) d t .
$$

Combining

$$
K_{\mu}(\lambda)=\frac{\pi}{2 \sin (\pi \mu)}\left[I_{-\mu}(\lambda)-I_{\mu}(\lambda)\right]
$$

and

$$
I_{\mu}(\lambda)=e^{-i \frac{\pi}{2} \mu} J_{\mu}\left(e^{i \frac{\pi}{2}} \lambda\right),
$$

the Bessel function $K$ can be written as

$$
K_{\mu}(\lambda)=\frac{1}{2} \Gamma(\mu) \Gamma(1-\mu)\left[e^{i \frac{\pi}{2} \mu} J_{-\mu}\left(e^{i \frac{\pi}{2}} \lambda\right)-e^{-i \frac{\pi}{2} \mu} J_{\mu}\left(e^{i \frac{\pi}{2}} \lambda\right)\right] .
$$

$C$ can then be computed using relation (6.688) of Ref. [33])

$$
\begin{aligned}
& \int_{0}^{\frac{\pi}{2}} J_{\mu}(z \sin t) \cos (x \cos t) d t=\frac{\pi}{2} J_{\frac{\mu}{2}}\left(y_{+}\right) J_{\frac{\mu}{2}}\left(y_{-}\right), \\
& \text {where } \quad y_{ \pm}=\frac{\sqrt{x^{2}+z^{2}} \pm x}{2} .
\end{aligned}
$$

In our case $\mu=-2 i \nu, x=\frac{q \cdot \underline{\rho}}{2}$ and $z=e^{i \frac{\pi}{2} \frac{q \rho}{2}}$. Defining $\Psi$ the angle $(\underline{q}, \underline{\rho})$, and computing $y_{ \pm}$,

$$
y_{ \pm}= \pm \frac{q \rho}{4} e^{ \pm i \Psi}
$$

one gets

$$
C=\frac{\pi}{2} \Gamma(-2 i \nu) \Gamma(1+2 i \nu)\left[J_{i \nu}\left(\frac{q \rho}{4} e^{i \Psi}\right) J_{i \nu}\left(\frac{q \rho}{4} e^{-i \Psi}\right)-J_{-i \nu}\left(\frac{q \rho}{4} e^{i \Psi}\right) J_{-i \nu}\left(\frac{q \rho}{4} e^{-i \Psi}\right)\right],
$$

where we have used the relation

$$
J_{\mu}\left(e^{i \pi} z\right)=e^{i \pi \mu} J_{\mu}(z)
$$

in order to get rid of the minus sign arising from the $y_{-}$contribution. Combining expressions (A.67) and (A.75), and using the expression (2.22) for $b_{n, \nu}$, one finally gets

$E_{q}^{0, \nu}(\rho)=\left(\frac{q}{2}\right)^{2 i \nu} 2^{-2 i \nu} \Gamma^{2}(1-i \nu)\left[J_{i \nu}\left(\frac{q \rho}{4} e^{i \Psi}\right) J_{i \nu}\left(\frac{q \rho}{4} e^{-i \Psi}\right)-J_{-i \nu}\left(\frac{q \rho}{4} e^{i \Psi}\right) J_{-i \nu}\left(\frac{q \rho}{4} e^{-i \Psi}\right)\right]$,

in agreement with the more general result (10) of Ref. [32]. 


\section{A.7 Properties of the three-points correlation functions $E^{n, \nu}$ and $E_{q}^{n, \nu}$}

In this appendix we derive various useful mathematical formulae for the functions $E^{n, \nu}$ and $E_{q}^{n, \nu}$. Let us first show that $E^{n, \nu}$ and $E^{-n,-\nu}$ are related by the following expression

$$
E^{n, \nu *}\left(\rho_{10}, \rho_{20}\right)=\frac{b_{n, \nu}^{*}}{a_{n, \nu}} \int d^{2} \rho_{0^{\prime}} E^{n, \nu}\left(\rho_{10^{\prime}}, \rho_{20^{\prime}}\right)\left|\rho_{00^{\prime}}\right|^{-2+4 i \nu}\left(\frac{\rho_{0^{\prime} 0}^{*}}{\rho_{0^{\prime} 0}}\right)^{n}(-1)^{n},
$$

which corrects formula (A.12) of [29]. Consider

$$
T=\int d^{2} \rho_{0^{\prime}} E^{n, \nu}\left(\rho_{10^{\prime}}, \rho_{20^{\prime}}\right)\left|\rho_{00^{\prime}}\right|^{-2+4 i \nu}\left(\frac{\rho_{0^{\prime} 0}^{*}}{\rho_{0^{\prime} 0}}\right)^{n} .
$$

Using conformal invariance, we can take $\rho_{2} \rightarrow \infty$ in order to simplify the calculation and restore the $\rho_{2}$ dependence afterwards by requiring the correct conformal transformation property. $T$ then reads

$$
\begin{aligned}
T & =\int d^{2} \rho_{0^{\prime}}\left(\frac{1}{\rho_{10^{\prime}}^{*}}\right)^{n}\left|\rho_{10^{\prime}}\right|^{n}\left|\rho_{00^{\prime}}\right|^{-2+4 i \nu}\left(\frac{\rho_{0^{\prime} 0}^{*}}{\rho_{00^{\prime}}}\right)^{n}\left|\frac{1}{\rho_{10^{\prime}}^{*}}\right|^{1+2 i \nu} \\
& =\int d^{2} R^{\prime}\left(\frac{R^{\prime}+\frac{\rho}{2}}{R^{\prime *}+\frac{\rho^{*}}{2}}\right)^{\frac{n}{2}}\left|R^{\prime}-R\right|^{-2+4 i \nu}\left(\frac{R^{\prime *}-R^{*}}{R^{\prime}-R}\right)^{n}\left|\frac{1}{R^{\prime}+\frac{\rho}{2}}\right|^{1+2 i \nu},
\end{aligned}
$$

where $\rho_{10}=R+\frac{\rho}{2}, \rho_{20}=R-\frac{\rho}{2}, \rho_{10^{\prime}}=R^{\prime}+\frac{\rho}{2}$ and $\rho_{20^{\prime}}=R^{\prime}-\frac{\rho}{2}$. After performing the change of variable $R^{\prime \prime}=R^{\prime}-R$ and introducing $R^{\prime \prime}=\left(R+\frac{\rho}{2}\right) z, T$ reads

$$
T=\left(\frac{R^{*}+\frac{\rho^{*}}{2}}{R^{*}+\frac{\rho}{2}}\right)^{\frac{n}{2}}\left|R+\frac{\rho}{2}\right|^{-1+2 i \nu} K
$$

where

$$
K=\int d^{2} z\left(\frac{z+1}{z^{*}+1}\right)^{\frac{n}{2}}|z|^{-2+4 i \nu}\left(\frac{z^{*}}{z}\right)^{n}\left|\frac{1}{z+1}\right|^{1+2 i \nu} .
$$

Using the techniques developped in Ref. [44], this integral can be computed after performing a Wick rotation for the $y$ integration. The corresponding replacement $y \rightarrow i y e^{-2 i \epsilon}$ reads $z+z^{*}=\alpha+\beta$ and $z-z^{*}=(\alpha-\beta) e^{-2 i \epsilon}$ with $d^{2} z=d x d y=\frac{1}{2} d z d z^{*}=\frac{i}{2} d \alpha d \beta$. Separating the integration in $\alpha$ in three domains, the non-zero contribution is obtained for $\alpha \in[-1,0]$ due to the $i \epsilon$. Closing the integration contour for the $\beta$ integration around the singularity $\beta=0$, this yields

$$
\begin{aligned}
K & =\frac{i}{2} \int d \alpha d \beta \frac{(\alpha+1)^{|n|}}{[(\alpha+1)(\beta+1)+i \epsilon]^{\frac{|n|+1}{2}+i \nu}} \frac{\beta^{2|n|}}{(\alpha \beta+i \epsilon)^{|n|+1-2 i \nu}} \\
& =\int_{0}^{1} d \alpha(1-\alpha)^{\frac{|n|-1}{2}-i \nu} \alpha^{-|n|-1+2 i \nu} \sin (|n|+1-2 i \nu) \int_{0}^{+\infty} d \beta \frac{\beta^{|n|-1+2 i \nu}}{(\beta+1)^{\frac{|n|+1}{2}+i \nu}}
\end{aligned}
$$

where we have performed the change of variable $\alpha \rightarrow-\alpha$. These integrals lead to $\beta$ functions. After some straightforward calculations, one gets

$$
K=\frac{\pi}{2} \frac{1}{-i \nu+\frac{|n|}{2}} 2^{4 i \nu} \frac{\Gamma\left(-i \nu+\frac{|n|+1}{2}\right)}{\Gamma\left(i \nu+\frac{|n|+1}{2}\right)} \frac{\Gamma\left(i \nu+\frac{|n|}{2}\right)}{\Gamma\left(-i \nu+\frac{|n|}{2}\right)}(-1)^{n}=\frac{b_{n, \nu}}{2 \pi^{2}}(-1)^{n}=\frac{a_{n, \nu}}{b_{n, \nu}^{*}}(-1)^{n} .
$$


Combining formulae (A.78, A.81, A.84) and using

$$
E^{n, \nu *}\left(\rho_{10}, \rho_{20}\right)=\left(\frac{R^{*}+\frac{\rho^{*}}{2}}{R+\frac{\rho}{2}}\right)^{\frac{n}{2}}\left|R+\frac{\rho}{2}\right|^{-1+2 i \nu},
$$

one finally gets formula (A.78) after restoring the correct dependence in $\rho_{2}$.

Note that from Eq. (A.78) one can obtain the corresponding relation between $E_{q}^{n, \nu *}$ and $E_{q}^{n, \nu}$. Indeed, performing the Fourier transform of both sides, one gets

$$
\begin{aligned}
& E_{q}^{n, \nu *}\left(\rho_{1}-\rho_{2}\right)=\frac{2 \pi^{2}}{b_{n, \nu}^{*}} \int \frac{d^{2} \frac{\underline{\rho}_{1}+\underline{\rho}_{2}}{2}}{\left|\rho_{1}-\rho_{2}\right|} e^{-i \frac{1}{2}\left(\underline{\rho}_{10}+\underline{\rho}_{20}\right) \cdot \underline{q}} E^{n, \nu *}\left(\underline{\rho}_{10}, \underline{\rho}_{20}\right) \\
& =\frac{b_{n, \nu}}{a_{n, \nu}} \frac{2 \pi^{2}}{b_{n, \nu}} \int \frac{d^{2} \frac{\underline{\rho}_{1}+\underline{\rho}_{2}}{2}}{\left|\rho_{1}-\rho_{2}\right|} e^{-i \frac{1}{2}\left(\underline{\rho}_{10^{\prime}}+\underline{\rho}_{20^{\prime}}\right) \cdot \underline{q}} E^{n, \nu}\left(\underline{\rho}_{10^{\prime}}, \underline{\rho}_{20^{\prime}}\right) \\
& \times \int d^{2} \underline{\rho}_{0^{\prime}} e^{i\left(\underline{\rho}_{0}-\underline{\rho}_{0^{\prime}}\right) \cdot \underline{q}}\left|\rho_{00^{\prime}}\right|^{-2+4 i \nu}\left(\frac{\rho_{0^{\prime} 0}^{*}}{\rho_{0^{\prime} 0}}\right)^{n}(-1)^{n} \\
& =\frac{b_{n, \nu}}{a_{n, \nu}} E_{-q}^{n, \nu}\left(\rho_{1}-\rho_{2}\right) \frac{b_{n, \nu}^{*}}{2 \pi^{2}}|q|^{-4 i \nu}\left(\frac{q^{*}}{q}\right)^{n} e^{-i \delta(n, \nu)},
\end{aligned}
$$

where the last integral is computed performing the integration $d^{2} \underline{\rho}_{0^{\prime}}=d^{2} \underline{\rho}_{0^{\prime} 0}$ with radial coordinates $\rho_{0^{\prime} 0}=\left|\rho_{0^{\prime} 0}\right| \exp (i \phi)$ (see the result (A.11) of Ref. 29]). Using the definition (2.21),

$$
\begin{aligned}
E_{-q}^{n, \nu}(\rho) & =\frac{2 \pi^{2}}{b_{n, \nu}} \int \frac{d^{2} \underline{R}}{|\rho|} e^{-i \underline{q} \cdot \underline{R}} E^{n, \nu}\left(R+\frac{\rho}{2}, R-\frac{\rho}{2}\right)=\frac{2 \pi^{2}}{b_{n, \nu}} \int \frac{d^{2} \underline{R}}{|\rho|} e^{i \underline{q} \cdot \underline{R}} E^{n, \nu}\left(-R+\frac{\rho}{2},-R-\frac{\rho}{2}\right) \\
& =\frac{2 \pi^{2}}{b_{n, \nu}} \int \frac{d^{2} \underline{R}}{|\rho|} e^{i \underline{q} \cdot \underline{R}} E^{n, \nu}\left(R+\frac{\rho}{2}, R-\frac{\rho}{2}\right)=E_{q}^{n, \nu}(\rho),
\end{aligned}
$$

where we have used the fact that

$$
E^{n, \nu}\left(\rho_{1}, \rho_{2}\right)=(-1)^{n} E^{n, \nu}\left(\rho_{2}, \rho_{1}\right)=E^{n, \nu}\left(-\rho_{2},-\rho_{1}\right) .
$$

Thus, using formula (2.38), one finally gets from Eq. (A.86)

$$
E_{q}^{n, \nu *}(\rho)=|q|^{-4 i \nu}\left(\frac{q^{*}}{q}\right)^{n} e^{-i \delta(n, \nu)} E_{q}^{n, \nu}(\rho),
$$

in agreement with formula (A.15) of Ref. 29].

Note that the function $E_{q}^{n, \nu}$ also possesses the following property

$$
\begin{aligned}
E_{q}^{n, \nu *}(\rho) & =\frac{2 \pi^{2}}{b_{n, \nu}^{*}} \int \frac{d^{2} \underline{R}}{|\rho|} e^{-i \underline{q} \cdot \underline{R}} E^{n, \nu *}\left(R+\frac{\rho}{2}, R-\frac{\rho}{2}\right) \\
& =\frac{2 \pi^{2}}{b_{-n,-\nu}} \int \frac{d^{2} \underline{R}}{|\rho|} e^{i \underline{q} \cdot \underline{R}} E^{n, \nu *}\left(-R+\frac{\rho}{2},-R-\frac{\rho}{2}\right)=E_{q}^{-n,-\nu}(\rho),
\end{aligned}
$$

where we have used the relation

$$
E^{n, \nu *}\left(\rho_{1}, \rho_{2}\right)=E^{-n,-\nu}\left(-\rho_{2},-\rho_{1}\right) .
$$


Let us now prove the following orthonormalisation property for the three-points correlation functions $E_{q}^{n, \nu}$

$$
\int \frac{d^{2} \underline{x}_{1}^{\prime}}{2 \pi x_{1}^{\prime 2}} E_{q}^{n_{1}, \nu_{1}}\left(x_{1}^{\prime}\right) E_{q}^{n, \nu *}\left(x_{1}^{\prime}\right)=\pi \delta_{n_{1}, n} \delta\left(\nu_{1}-\nu\right)+\pi \delta_{n_{1},-n} \delta\left(\nu_{1}+\nu\right)\left(\frac{q}{q^{*}}\right)^{n_{1}}\left|q^{2}\right|^{2 i \nu_{1}} e^{i \delta\left(n_{1}, \nu_{1}\right)} .
$$

Using the definition (2.21), the left-handside reads

$$
\begin{aligned}
& \int \frac{d^{2} \underline{x}_{1}^{\prime}}{2 \pi x_{1}^{\prime 2}} E_{q}^{n_{1}, \nu_{1}}\left(x_{1}^{\prime}\right) E_{q}^{n, \nu *}\left(x_{1}^{\prime}\right)=\int \frac{d^{2} \underline{x}_{1}^{\prime}}{2 \pi x_{1}^{\prime 2}} \frac{2 \pi^{2}}{b_{n, \nu}^{*}} \frac{2 \pi^{2}}{b_{n_{1}, \nu_{1}}} \\
& \times \int \frac{d^{2} \underline{R} d^{2} \underline{R^{\prime}}}{x_{1}^{\prime 2}} e^{i q} \cdot\left(\underline{R}-\underline{R^{\prime}}\right) E^{n_{1}, \nu_{1}}\left(R+\frac{x_{1}^{\prime}}{2}, R-\frac{x_{1}^{\prime}}{2}\right) E^{n, \nu *}\left(R^{\prime}+\frac{x_{1}^{\prime}}{2}, R^{\prime}-\frac{x_{1}^{\prime}}{2}\right) .
\end{aligned}
$$

Performing the changes of variables

$$
\rho_{1}-\rho_{0}=R+\frac{x_{1}^{\prime}}{2}, \quad \rho_{2}-\rho_{0}=R-\frac{x_{1}^{\prime}}{2}, \quad \rho_{1}-\rho_{0^{\prime}}=R^{\prime}+\frac{x_{1}^{\prime}}{2}, \quad \rho_{2}-\rho_{0^{\prime}}=R^{\prime}-\frac{x_{1}^{\prime}}{2},
$$

and using the equality

$$
d^{2} \underline{R} d^{2} \underline{R}^{\prime} d^{2} \underline{x}_{1}^{\prime}=d^{2} \underline{\rho}_{1} d^{2} \underline{\rho}_{2} d^{2} \underline{\rho}_{0^{\prime}}
$$

one can apply the orthonormalization condition (2.48), which yields

$$
\begin{gathered}
\int \frac{d^{2} \underline{x}_{1}^{\prime}}{2 \pi x_{1}^{\prime 2}} E_{q}^{n_{1}, \nu_{1}}\left(x_{1}^{\prime}\right) E_{q}^{n, \nu *}\left(x_{1}^{\prime}\right)=\frac{2 \pi^{2}}{b_{n, \nu}^{*}} \frac{2 \pi^{2}}{b_{n_{1}, \nu_{1}}} \frac{1}{2 \pi}\left[a_{n, \nu} \delta_{n_{1}, n} \delta\left(\nu_{1}-\nu\right) \int d^{2} \underline{\rho}_{0^{\prime}} e^{i \underline{q} \cdot \underline{\rho}_{0^{\prime} 0}} \delta^{2}\left(\rho_{0^{\prime} 0}\right)\right. \\
\left.+b_{n_{1}, \nu_{1}} \delta_{n_{1},-n} \delta\left(\nu+\nu_{1}\right)(-1)^{n} \int d^{2} \underline{\rho}_{00^{\prime}}\left|\rho_{00^{\prime}}\right|^{-2-4 i \nu}\left(\frac{\rho_{00^{\prime}}}{\rho_{00^{\prime}}^{*}}\right)^{n_{1}} e^{i \underline{q} \cdot \underline{\rho}_{0^{\prime}}}\right] .
\end{gathered}
$$

Using the result (A.11) of Ref. [29] for the last integral, one finally gets the expected result (A.92).

\section{A.8 Calculation of $F^{(1)}$ in the mixed representation}

In this appendix we show how to get the onium-onium scattering amplitude at fixed impact parameter using the mixed representation for the dipole distribution (see Eq. (2.23)) and for the elementary dipole-dipole cross-section (see Eq. (A.30)). Using the Fourier representation of the $\delta$ distribution, formula (2.7) reads

$$
\begin{aligned}
& F^{(1)}\left(x_{1}, x_{2}, \tilde{Y}, b\right)=-\frac{1}{2} \int \frac{d^{2} \underline{x}_{1}^{\prime}}{2 \pi x_{1}^{\prime 2}} \frac{d^{2} \underline{x}_{2}^{\prime}}{2 \pi x_{2}^{\prime 2}} d^{2} \underline{b}_{1} d^{2} \underline{b}_{2} d^{2}\left(\underline{b}_{2}^{\prime}-\underline{b}_{1}^{\prime}\right) \int \frac{d^{2} \underline{q}}{(2 \pi)^{2}} e^{i q} \cdot\left(\underline{b}_{1}-\underline{b}_{2}-\underline{b}_{1}^{\prime}+\underline{b}_{2}^{\prime}-\underline{b}\right) \\
& \times n\left(\underline{x}_{1}, \underline{x}_{1}^{\prime}, \underline{b}_{1}, \tilde{Y}_{1}\right) n\left(\underline{x}_{2}, \underline{x}_{2}^{\prime}, \underline{b}_{2}, \tilde{Y}_{2}\right) \sigma_{D D}\left(\underline{x}_{1}^{\prime}, \underline{x}_{2}^{\prime}, \underline{b}_{1}^{\prime}-\underline{b}_{2}^{\prime}\right) .
\end{aligned}
$$

Combining this expression with formulae (2.23) and (A.30), one gets

$$
\begin{aligned}
& F^{(1)}\left(x_{1}, x_{2}, \tilde{Y}, b\right)=-\frac{1}{2} \int \frac{d^{2} \underline{q}}{(2 \pi)^{2}} e^{-i \underline{q} \cdot \underline{b}} \int \frac{d^{2} \underline{x}_{1}^{\prime}}{2 \pi x_{1}^{\prime 2}} \frac{d^{2} \underline{x}_{2}^{\prime}}{2 \pi x_{2}^{\prime 2}} \sum_{n_{1}=-\infty}^{+\infty} \int_{-\infty}^{+\infty} \frac{d \nu_{1}}{2 \pi} \frac{x_{1}}{x_{1}^{\prime}} E_{q}^{n_{1}, \nu_{1} *}\left(x_{1}\right) E_{q}^{n_{1}, \nu_{1}}\left(x_{1}^{\prime}\right) \\
& \times \sum_{n_{2}=-\infty}^{+\infty} \int_{-\infty}^{+\infty} \frac{d \nu_{2}}{2 \pi} \frac{x_{2}}{x_{2}^{\prime}} E_{q}^{n_{2}, \nu_{2} *}\left(x_{2}\right) E_{q}^{n_{2}, \nu_{2}}\left(x_{2}^{\prime}\right) 2 \alpha_{s}^{2} \frac{x_{1}^{\prime} x_{2}^{\prime}}{16} \sum_{n=-\infty}^{+\infty} \int_{-\infty}^{+\infty} d \nu \frac{1+(-1)^{n}}{\left(\nu^{2}+\left(\frac{n-1}{2}\right)^{2}\right)\left(\nu^{2}+\left(\frac{n+1}{2}\right)^{2}\right)} \\
& \times E_{q}^{n, \nu *}\left(x_{1}^{\prime}\right) E_{q}^{n, \nu}\left(x_{2}^{\prime}\right) \exp \left(\frac{2 \alpha_{s}^{2} N_{c}}{\pi}\left(\chi\left(n_{1}, \nu_{1}\right) \tilde{Y}_{1}+\chi(n, \nu) \tilde{Y}_{2}\right)\right)
\end{aligned}
$$


From the property (A.90), one has $E_{q}^{n_{2}, \nu_{2}}=E_{q}^{-n_{2},-\nu_{2} *}$. We can now apply the orthonormalization condition (A.92), which yields

$$
\begin{gathered}
F^{(1)}\left(x_{1}, x_{2}, \tilde{Y}, b\right)=-\frac{\pi \alpha_{s}^{2} x_{1} x_{2}}{8} \int \frac{d^{2} \underline{q}}{(2 \pi)^{2}} e^{-i \underline{q} \cdot \underline{b}} \sum_{n=-\infty}^{+\infty} \int_{-\infty}^{+\infty} d \nu E_{q}^{n, \nu *}\left(x_{1}\right) E_{q}^{n, \nu}\left(x_{2}\right) \\
\times \frac{1+(-1)^{n}}{\left(\nu^{2}+\left(\frac{n-1}{2}\right)^{2}\right)\left(\nu^{2}+\left(\frac{n+1}{2}\right)^{2}\right)} \exp \left(\frac{2 \alpha_{s} N_{c}}{\pi}\left(\chi\left(n_{1}, \nu_{1}\right) \tilde{Y}_{1}+\chi(n, \nu) \tilde{Y}_{2}\right)\right) .
\end{gathered}
$$

From the expression of the dipole density (2.23), this finally reads

$$
F_{\{n, \nu\}}^{(1)}\left(\underline{x}_{1}, \underline{x}_{2}, \underline{b}\right)=-\frac{\pi \alpha_{s}^{2} x_{2}^{2}}{8} \frac{1+(-1)^{n}}{\left(\nu^{2}+\left(\frac{n-1}{2}\right)^{2}\right)\left(\nu^{2}+\left(\frac{n+1}{2}\right)^{2}\right)} n_{\{n, \nu\}}\left(\underline{x}_{1}, \underline{x}_{2}, \underline{b}\right) .
$$

which is identical to Eq. (2.58) as expected. 


\section{References}

[1] H1 Coll., S. Aid et al., A Measurement and QCD Analysis of the Proton Structure Function $F_{2}\left(x, Q^{2}\right)$ at HERA, Nucl. Phys. B470 (1996) 3.

[2] ZEUS Coll., M. Derrick et al., Measurement of the $F_{2}$ structure function in deep inelastic $e^{ \pm} p$ scattering using 1994 data from the ZEUS detector at HERA, Zeit. für Phys., C72 (1996) 399-424.

(1972) 438 and 675.

[3] E.A. Kuraev, L.N. Lipatov and V.S. Fadin, On the Pomeranchuk Singularity in Asymptotically Free Theory, Phys. Lett. B60 (1975) 50-52; Multiregge processes in the Yang-Mills theory, Sov. Phys. JETP 44 (1976) 443-451; The Pomeranchuk Singularity in Non Abelian Gauge Theories, Sov. Phys. JETP 45 (1977) 199-204; Ya.Ya. Balitskii and L.N. Lipatov, The Pomeranchuk Singularity in Quantum Chromodynamics, Sov. J. Nucl. Phys. 28 (1978) $822-829$.

[4] J. Bartels, High-Energy Behaviour in a Non-Abelian Gauge Theory (II). First corrections to $T_{n \rightarrow m}$ beyond the leading $\ln s$ approximation, Nucl. Phys. B175 (1980) 365-401.

[5] T. Jaroszewicz, Infra-red divergences and Regge behaviour in QCD, Acta Phys. Pol. B11 (1980) 965-973.

[6] J. Kwiecinski and M. Praszalowicz, Three gluon integral equation and odd $C$ singlet Regge singularities in QCD, Phys. Lett. B94 (1980) 413-416.

[7] L.N. Lipatov, High-energy asymptotics of multicolor QCD and exactly solvable lattice models, Padova preprint DFPD-93-TH-70, Oct 1993; Asymptotic behaviour of multicolor QCD at high energies in connection with exactly solvable spin models, JETP Lett. 59 (1994) 596-599.

[8] L.D. Faddeev and G.P. Korchemsky, High energy QCD as a completely integrable model, Phys. Lett. B342 (1995) 311-322.

[9] G.P. Korchemsky, Bethe Ansatz for QCD Pomeron, Nucl. Phys. B 443 (1995) 255-304.

[10] G.P. Korchemsky, Quasiclassical QCD Pomeron, Nucl. Phys. B462 (1996) 333-388.

[11] Z. Maassarani and S. Wallon, Baxter Equation for the QCD Odderon, J. Phys. A: Math. Gen. 28 (1995) 6423-6434.

[12] R. Janik, Modular Invariance and the Odderon, Phys. Lett. B371 (1996) 293-297.

[13] J. Wosiek and R. Janik, Solution of the Odderon problem for arbitrary conformal weights, preprint TP-JU 21/96, hep-th/9610208.

[14] A.H. Mueller, Soft gluons in the infinite-momentum wave function and the BFKL Pomeron, Nucl. Phys. B415 (1994) 373-385.

[15] A.H. Mueller and B. Patel, Single and double Pomeron exchange and a dipole picture of high energy hard processes, Nucl. Phys. B425 (1994) 471-488.

[16] A.H. Mueller, Unitarity and the BFKL Pomeron, Nucl. Phys. B437 (1995) 107-126. 
[17] Z. Chen and A.H. Mueller, The dipole picture of high energy scattering, the BFKL equation and many gluon compound states, Nucl. Phys. B 451 (1995) 579-604.

[18] N.N. Nikolaev and B.G. Zakharov, Color Transparency and Scaling Properties of Nuclear Shadowing in Deep Inelastic Scattering, Zeit. für. Phys. C49 (1991) 607-618; Pomeron Structure Function and diffractive Dissociation of Virtual Photon in Perturbative QCD, Zeit. für Phys. C53 (1992) 331-346.

[19] N.N. Nikolaev, B.G. Zakharov and V.R. Zoller, The Spectrum and Solutions of the Generalized BFKL Equation for Total Cross-Section, Phys. Lett. B328 (1994) 486-494.

[20] S. Catani, M. Ciafaloni and F. Hautmann, Gluons Contributions to Small x Heavy Flavour Production, Phys. Lett. B242 (1990) 97; S. Catani, M. Ciafaloni and F. Hautmann, High energy factorization and small-x heavy flavour production, Nucl. Phys. B366 (1991) 135-188; S. Catani and F. Hautmann, Quark anomalous dimensions at small $x$, Phys. Lett. B315 (1993) 157-163; High-energy factorization and small-x deep inelastic scattering beyond leading order, Nucl. Phys. B427 (1994) 475-524; M. Ciafaloni, $k_{t}$ factorization vs. renormalisation group: a small-x consistency argument, Phys. Lett. B356 (1995) 74-78.

[21] J.C. Collins and R.K. Ellis, Heavy-Quark Production in Very High Energy Hadron Collisions, Nucl. Phys. B360 (1991) 3-30;

[22] E.M. Levin, M.G.Ryskin. Yu.M. Shabelskii and A.G. Shuvaev, Heavy-quark production in semi-hard nucleon interactions, Sov. J. Nucl. Phys 53 (1995) 657-667.

[23] H. Navelet, R. Peschanski and Ch. Royon, Deep Inelastic Onium Scattering, Phys. Lett. B366 (1996) 329-336.

[24] H. Navelet, R. Peschanski, Ch. Royon and S. Wallon, Proton structure function in the dipole picture of BFKL dynamics, Phys. Lett. 385 (1996) 357-364.

[25] S. Wallon, QCD dipole predictions for quark singlet, gluon and $F_{L} / F_{T}$ distributions at HERA, proceedings Moriond 1996, preprint SPhT T96/048, hep-ph/9610332.

[26] Y. Kovchegov, A.H. Mueller and S. Wallon, Unitarity Corrections and High Field Strengths in High Energy Hard Collisions, preprint CU-TP-825, DESY 97-072, hep-ph/9704369.

[27] G.P. Salam, Studies of Unitarity at small $x$ Using the Dipole Formulation, Nucl. Phys. B 461(1996) 512-538.

[28] A.H. Mueller and G.P. Salam, Large multiplicity fluctuations and saturation effects in onium collisions, Nucl. Phys B475 (1996) 293-320.

[29] L. N. Lipatov, The bare Pomeron in quantum chromodynamics, Sov. Phys. JETP 63 (5) (1986) 904-912.

[30] A.M. Polyakov, Conformal Symmetry of Critical Fluctuations, JETP let. 12 (1970) 381383.

[31] I.M. Gel'fand, M.I. Graev, N.Ya. Vilenkin, Generalized Functions (vol 5) Integral Geometry and Representation Theory, Academic Press, New York (1966); C. Itzykson, Quelques aspects de la théorie des groupes, Lectures notes for the Mathematical Physics School of Toulouse University (1974) (in french). 
[32] H. Navelet and R. Peschanski, Conformal invariance and the exact solution of BFKL equations, preprint SPhT 97/016, hep-ph/9703238.

[33] I.S. Gradshteyn and I.M. Ryzhik, Table of Integrals, Series, and Products, Academic Press, New York (1965).

[34] W. Magnus, F. Oberhettinger and R.P. Soni, Formula and Theorem for the Special Functions of Mathematical Physics, Springer-Verlag, New York (1966) p103.

[35] G. Salam, Thesis, Cambridge (1996).

[36] H. Navelet, R. Peschanski, Ch. Royon and S. Wallon, in preparation.

[37] A.H. Mueller Parton distributions at very small-x values, Nucl. Phys. B (Proc. Suppl.) 18C (1991) 125-132; J. Bartels, A. De Roeck and M. Loewe, Measurement of hot spots inside the proton at HERA and LEP/LHC, Zeit. für Phys. C54 (1992) 635-642; J. Bartels, M. Besançon, A. De Roeck and J. Kurzhoefer, Measurement of hot spots at HERA, in Proceedings of the HERA Workshop 1991, eds. W. Buchmueller and G. Ingelman, 203-213; J. Kwiecinski, A.D. Martin and P.J. Sutton, Probing the Lipatov singular small $x$ behavior at HERA, Phys. Lett. B287 (1992) 254-258; Deep inelastic events containing a measured jet as a probe of QCD behavior at small x, Phys. Rev D46 (1992) 921-930; W.K. Tang, The structure function $\nu W(2)$ of hot spots at HERA, Phys. Lett. bf 278 (1991) 363-366.

[38] A.H. Mueller and H. Navelet, An Inclusive Minijet Cross Section and the Bare Pomeron in QCD, Nucl. Phys. B282 (1987) 727-744.

[39] J. Bartels, A. De Roeck and H. Lotter, The $\gamma^{*} \gamma^{*}$ Total Cross Section and the BFKL Pomeron at $e^{+} e^{-}$Colliders, Phys. Lett. B389 (1996) 742-748; S.J. Brodsky, F. Hautmann and D.E. Soper, Probing the QCD Pomeron in $e^{+} e^{-}$collisions, Phys. Rev. Lett. 78 (1997) 803-806.

[40] A. De Roeck, Ch. Royon and S. Wallon, to appear.

[41] L.N. Lipatov, Pomeron in quantum chromodynamics, in "Perturbative QCD", pp. 411-489, ed. A.H. Mueller, World Scientific, Singapore, 1989.

[42] Y.V. Kovchegov, Non-Abelian Weizsäcker-Williams field and a two-dimensional effective color charge density for a very large nucleus, Phys. Rev. D54 (1996) 5463-5469.

[43] A. Erdélyi, W. Magnus, F. Oberhettinger, F.G. Tricomi, Bateman Manuscript Project, McGraw-Hill, New York (1953) p189.

[44] Vl.S. Dotsenko and V.A. Fateev, Conformal algebra and multipoint correlation functions in 2 statistical models, Nucl. Phys. B240 [FS12] (1984) 312-348; Vl.S. Dotsenko, Lectures on conformal field theory, Advanced Studies in Pure Mathematics 16 (1988) 123-170. 